\title{
Motivational Factors Contributing to the Choice of Agriculturally Related Careers
}

\author{
Lacey J. Sims \\ West Virginia University
}

Follow this and additional works at: https://researchrepository.wvu.edu/etd

\section{Recommended Citation}

Sims, Lacey J., "Motivational Factors Contributing to the Choice of Agriculturally Related Careers" (2014). Graduate Theses, Dissertations, and Problem Reports. 350.

https://researchrepository.wvu.edu/etd/350

This Thesis is protected by copyright and/or related rights. It has been brought to you by the The Research Repository @ WVU with permission from the rights-holder(s). You are free to use this Thesis in any way that is permitted by the copyright and related rights legislation that applies to your use. For other uses you must obtain permission from the rights-holder(s) directly, unless additional rights are indicated by a Creative Commons license in the record and/ or on the work itself. This Thesis has been accepted for inclusion in WVU Graduate Theses, Dissertations, and Problem Reports collection by an authorized administrator of The Research Repository @ WVU. For more information, please contact researchrepository@mail.wvu.edu. 
Motivational Factors Contributing to the Choice of Agriculturally Related Careers

\author{
Lacey J. Sims
}

Thesis submitted to the Davis College of Agriculture, Natural Resources and Design at West Virginia University in partial fulfilment of the requirements for the degree of

\author{
Master of Science \\ in \\ Agricultural and Extension Education \\ Deborah A. Boone, Ph.D., Chair \\ Harry N. Boone, Jr., Ph.D. \\ Jean M. Woloshuk, Ed.D. \\ Division of Resource Management \\ Morgantown, West Virginia \\ 2014
}

Keywords: Motivation, Agriculture, Careers 


\section{ABSTRACT \\ Motivational Factors Contributing to the Choice of Agriculturally Related Careers}

This study examined the motivational factors of college students who have chosen to study in an agriculturally related field. A descriptive research design using an online questionnaire was used for this study to determine factors which influenced career choice. Likert scale items were used to determine how motivating certain factors were for respondents in influencing their decision to study in an agriculturally related field. The Likert items were divided into five constructs: family influence, self-motivation, experience, use of skills, and career opportunities. Items within the five constructs were further analyzed to discover the top five motivational factors identified in each of the constructs. The top five "family influences" were found to be: teachers, agricultural professionals, family encouragement, parents, and the desire to do something the family has never done. The top influences for the construct of "self-motivation" were: a drive to succeed, highly self-motivated, creating innovative ways to improve the quality of life, finding new ways to perform career related tasks, and the desire to work with ones hands. The top five items from the "experience" construct that the respondents felt were highly motivational for their situations included: worked on a farm, prior experience in agriculture, worked in an agricultural business, agricultural courses in high school, and participation in National FFA convention. The top five factors comprising the construct "use of skills" were: enjoyable career options, variety of challenging activities, opportunities to work with knowledgeable professionals, use of verbal communication skills, and use of problem solving strategies. Thirteen items comprised the construct "career opportunities". The top five items identified were: multiple opportunities in agriculture, career opportunities associated with degree, employment directly after college, number of job locations, and the size of the industry. This study also found relationships between pursuing an agriculturally related career and life experiences, such as working on a farm or in an agricultural business or relatives who have worked on a farm or in an agricultural business. Relationships also existed between pursuing agricultural careers and high school agricultural courses as well as pursuing an agriculturally related career and college agricultural organization involvement. 


\section{DEDICATION}

"For I know the plans I have for you," declares the LORD, "plans to prosper you and not to harm you, plans to give you hope and a future. ${ }^{12}$ Then you will call on me and come and pray to me, and I will listen to you. ${ }^{13}$ You will seek me and find me when you seek me with all your heart."

$\sim$ Jeremiah 29: $11-13$

The heart of man plans his way, but the Lord establishes his steps.

$\sim$ Proverbs 16:9

Commit your work to the Lord, and your plans will be established. The Lord has made everything for its purpose, even the wicked for the day of trouble.

$\sim$ Proverbs 16:3 - 4 


\section{ACKNOWLEDGEMENTS}

I want to thank mom and dad for pushing me to continue my education. You knew working on a thesis to obtain a Masters was the farthest plan from my mind, yet you convinced me to do it anyway. Thank you. You have been an endless support system while working through my Masters, and I cannot thank you enough for the prayers... we did it!

A special thanks to my committee members: Dr. Jean Woloshuk, Dr. Debby Boone, and Dr. Harry Boone.

Dr. Debby, I appreciate, more than I can express, the stream of answers to endless questions over the years. I am thankful for your commitment and truly going above and beyond as a professor.

Dr. Jean: Thank you for serving on my committee. I appreciate your expert opinion during our discussions. I am thankful for the opportunity to have worked with you on this project.

Dr. Harry, I appreciate your thoroughness throughout the stages of this thesis. Your tendency to hold students to the highest standards is highly appreciated. You have always said you wanted your students to do well... Thank you for allowing the time for me to succeed.

Last, but of course not least... the friends who have supported me over the past year. While attempting to finish the program in record time, you still managed to stick by me... I am so thankful for each of you! I am thankful for the smiles and words of encouragement throughout the year. I am most thankful for the laughs each of you have brought my way during one time or another. If you ever need any of the above, you know where to find me... I would love to return the favor! 


\section{TABLE OF CONTENTS}

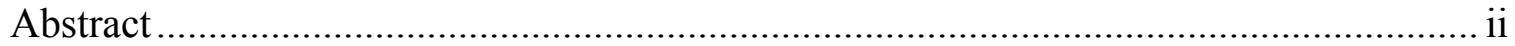

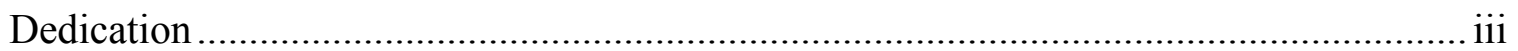

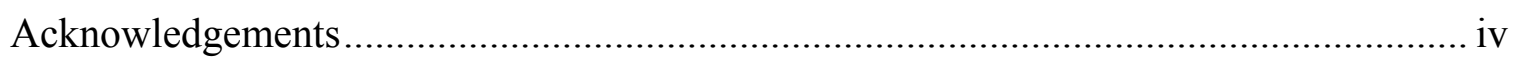

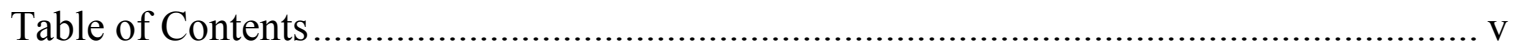

List of Tables ................................................................................................... viii

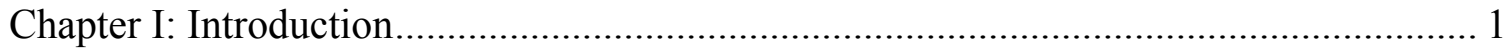

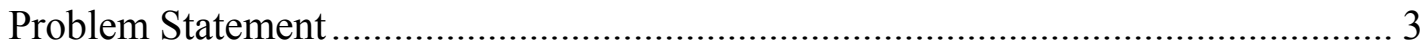

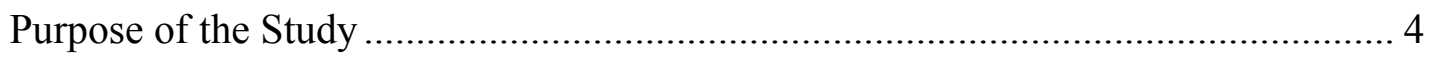

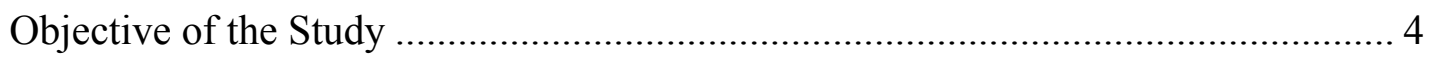

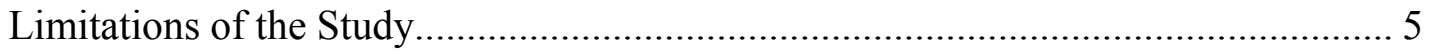

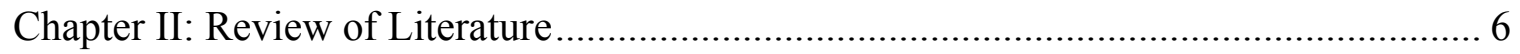

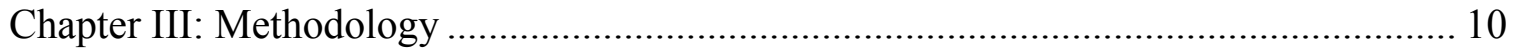

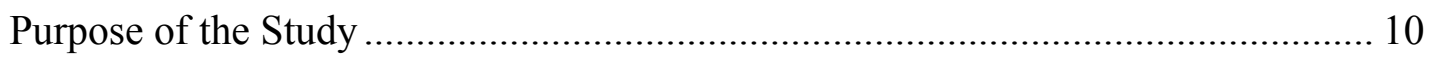

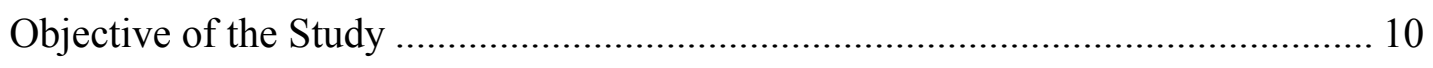

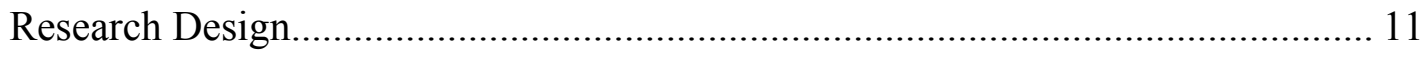

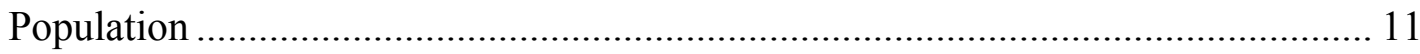

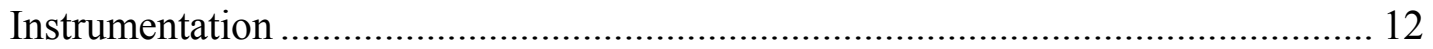

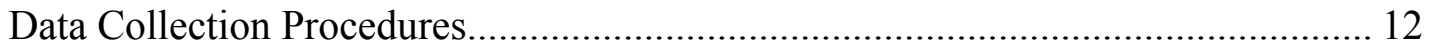

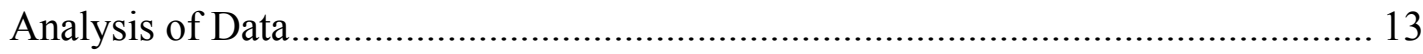

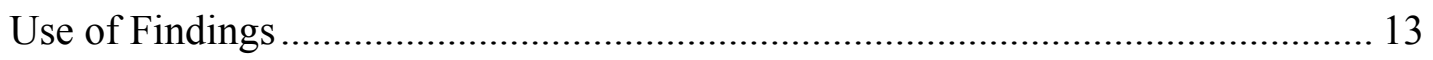

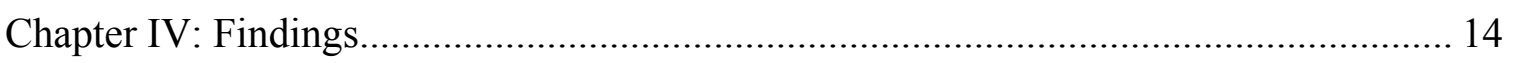

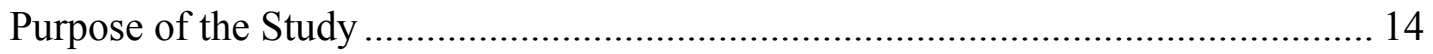

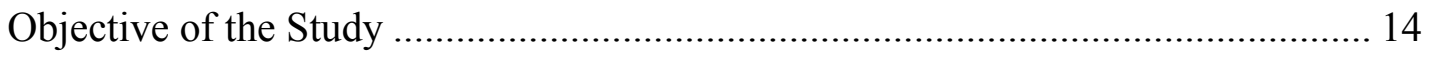

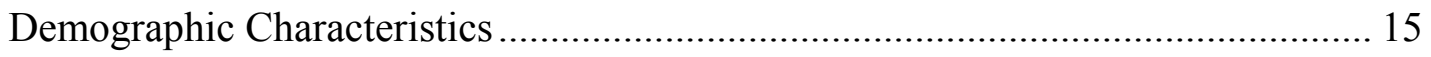

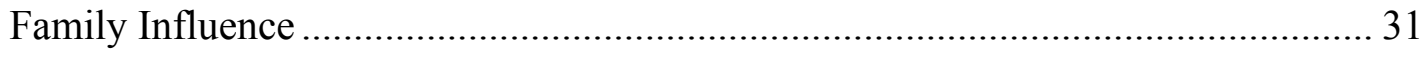

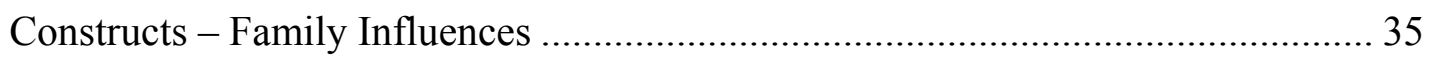

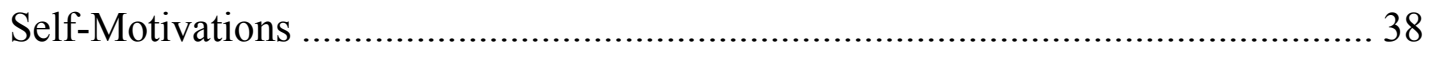

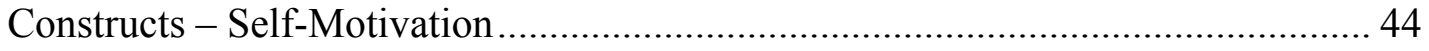

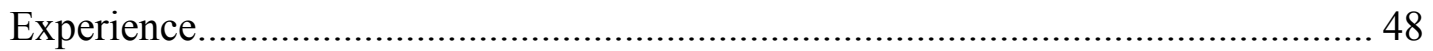

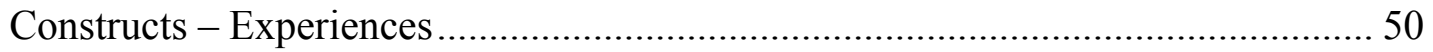

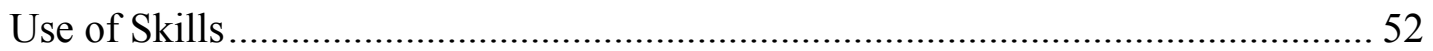




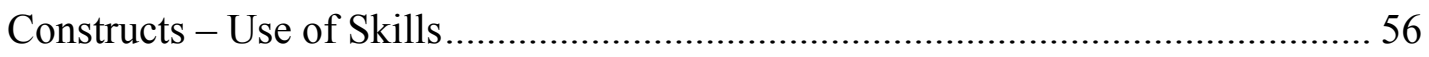

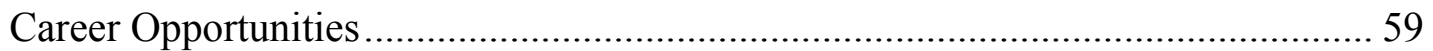

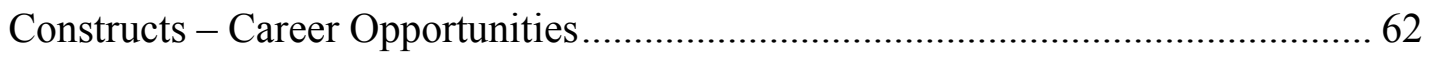

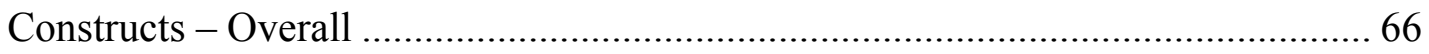

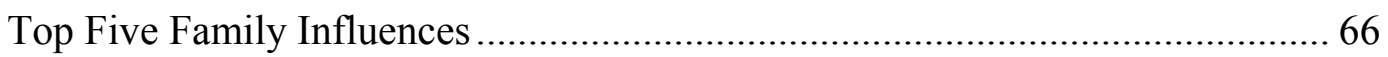

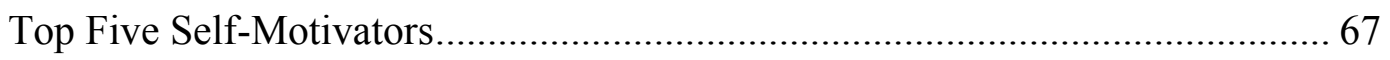

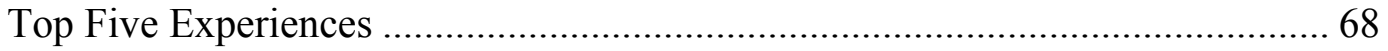

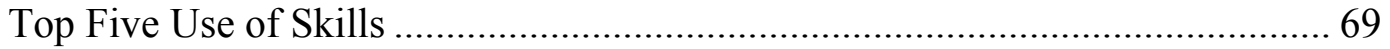

Top Five Career Opportunities …………………......................................... 70

Relationship between having worked on a Farm or Ranch and Pursuing an Agriculturally Related Career ...........................................................................

Relationship between having worked in an Agricultural Business and Pursuing an Agriculturally Related Career..

Relationship between having Relatives Working on a Farm or Ranch and Pursuing an Agriculturally Related Career ................................................................

Relationship between having Relatives Working in an Agricultural Business and Pursuing an Agriculturally Related Career ...............................................................78

Relationship between Taking High School Agricultural Course and Pursuing an Agriculturally Related Career ....

Relationship between College Agriculturally Related Organization Involvement and Pursuing an Agriculturally Related Career........................................................82

Relationship between Gender and Pursuing an Agriculturally Related Career .........84

Chapter V: Summary, Conclusions, and Recommendations ………………………...... 86

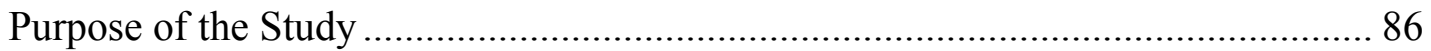

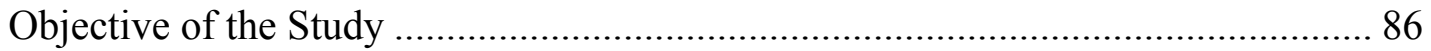

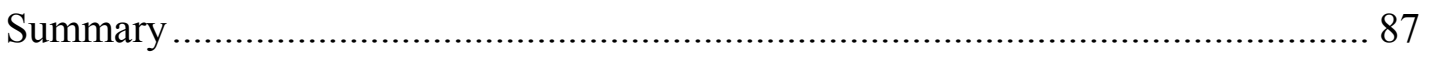

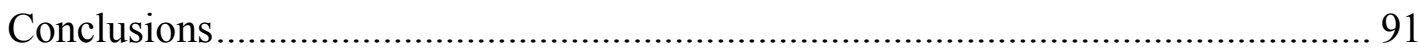

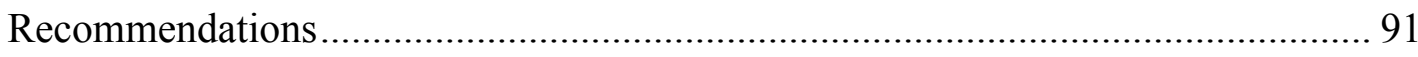

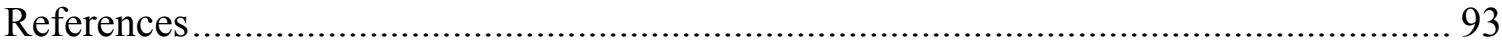

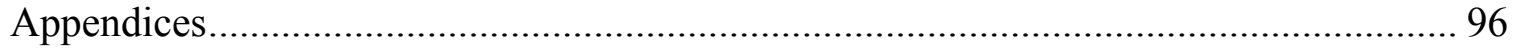

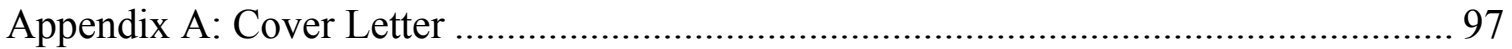

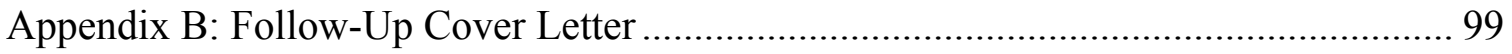

Appendix C: Third Follow-Up Cover Letter ……………………………………..... 101

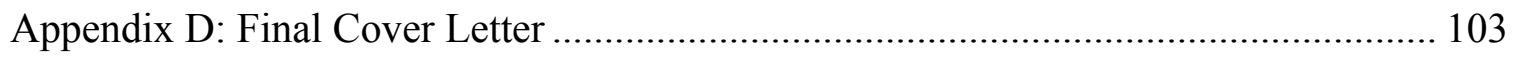




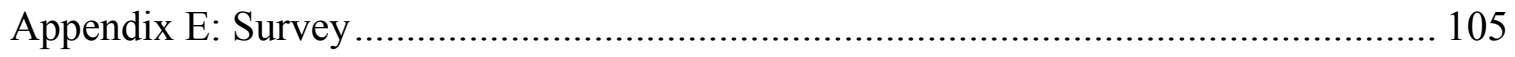

Appendix F: What is your mother/ female guardian's occupation? ............................. 117

Appendix G: What is your father/ male guardian's occupation?............................... 121

Appendix H: Agriculturally related organizations involved in as a youth. .................. 126

Appendix I: Study focus at West Virginia University? ............................................ 128

Appendix J: Other comments, questions, or concerns? ............................................ 130

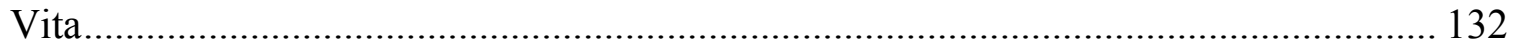




\section{LIST OF TABLES}

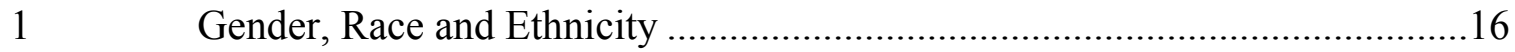

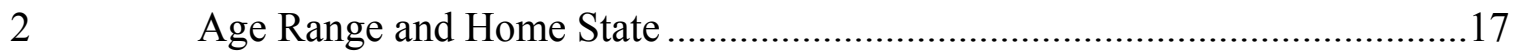

3 Place of Residency, Student Status, Grade Point Average ...............................19

$4 \quad$ Participation in Organizations as a Youth ..................................................21

$5 \quad$ Study Focus at West Virginia University ……………………………….......22

6 Pursuing Agriculturally Related Careers and Satisfaction with Career

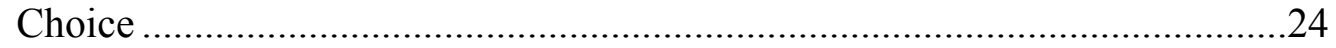

$7 \quad$ Estimated Starting Salary of Career Choice ……………………………........25

$8 \quad$ Type of Agricultural Background and Experiences.......................................26

$9 \quad$ Mother/ Female Guardian Occupations ....................................................28

$10 \quad$ Father/ Male Guardians Occupation ………….............................................30

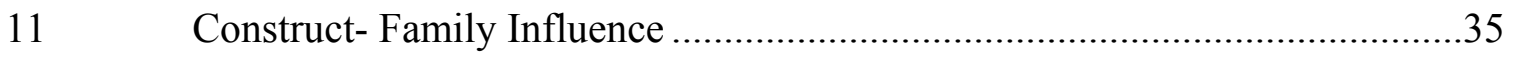

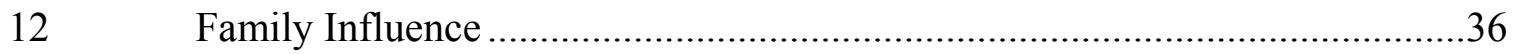

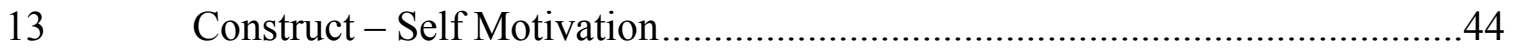

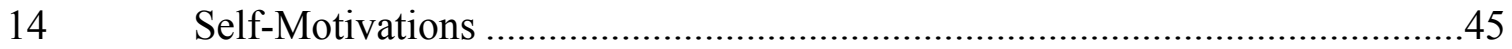

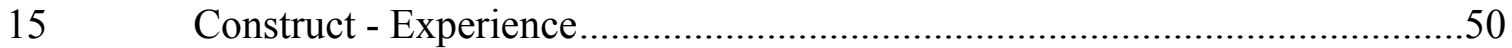

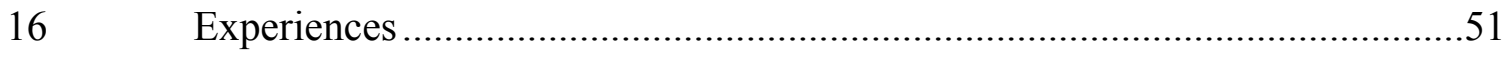

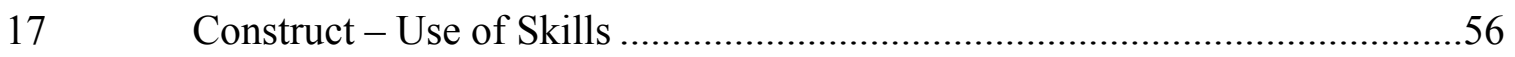

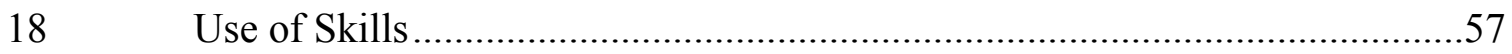

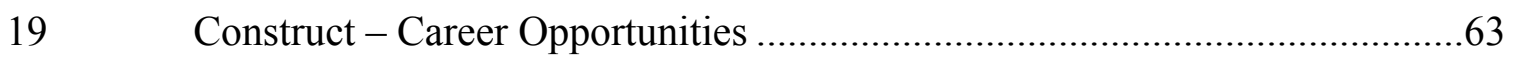

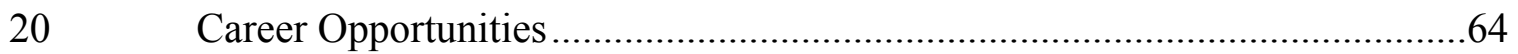

$21 \quad$ Construct - Overall Summary ……………………................................66

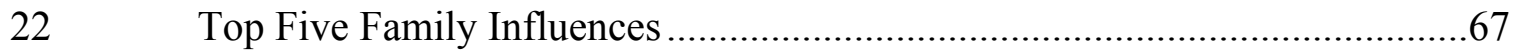

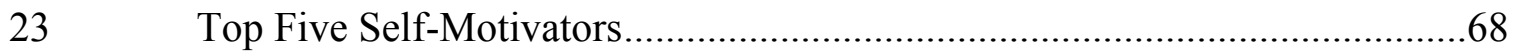


27 Chi Square Analysis- Worked on a Farm or Ranch and Pursuing an Agriculturally Related Career.

28 Chi-square Analysis: Worked in Agricultural Business and Pursuing an Agriculturally Related Career .

29 Chi-square Analysis: Relatives Working on a Farm or Ranch and Pursing an Agriculturally Related Career....

30 Chi-Square Analysis: Relatives Working in an Agricultural Business and Pursing an Agriculturally Related Career ....

31 Chi-Square Analysis: High School Agricultural Courses and Pursuing an Agriculturally Related Career.

32 Chi-Square Analysis: College Agricultural Organization Involvement and Pursuing an Agriculturally Related Career ....

33 Chi-Square Analysis: Gender and Pursing an Agriculturally Related Career 


\section{CHAPTER I}

\section{Introduction}

Multiple motivational factors contribute to choosing careers. These factors are individual in nature and not career specific, choosing specific careers is an individual choice. A survey conducted by $\mathrm{Ng}$ (2008) identified 10 motivational factors, including: appreciation or recognition for a job well done, being in the know about company matters, an understanding attitude from the management, job security, good wages, interesting work, career advancement opportunities, loyalty from management, good working conditions, and tactful discipline. Llopis (2012) created a list of nine traits that motivate employees to achieve in the workplace. Those include: trustworthy leadership, being relevant, proving others wrong, career advancement, reaching for goals, stable future, self-indulgence, making an impact, and happiness (Llopis, 2012). Several studies have reported that parents, family, teachers, counselors, and/or friends are highly motivating factors when deciding on career options (Bowen \& Lee, 1984; Cole, 1985; Donnermeyer \& Kreps, 1994; Faulks, 1969; Findlay \& Rawls, 1984; Miller, Williams, \& Sprouse, 1984; Reis \& Kahler, 1997; Sutpin \& Newsom-Stewart, 1995; Wildman \& Torres, 2001).

Although there are multiple motivational factors associated with choosing careers, the order in which these motivational factors are presented are individualistic in nature. Humans have multiple needs to be met. Food, shelter, and security are just a small sample of those needs. There are various theories about human needs as well as how those needs are fulfilled. Maslow (1943) proposed the Hierarchy of Needs Theory, which looks at the development of five hierarchal levels: Physiological, Safety, Love, 
Esteem, and Self-Actualization. Maslow (1943) believes that the lowest unmet need will need to be met in order to move up the ladder to meet the next unmet need. For example, a hungry person (physiological need) will not worry about safety until he has eaten. According to Maslow, a need must be unmet to become motivating and people are motivated by the lowest-level need that is unmet (Spector, 2003).

Maslow recognized, however, that there can be exceptions to the hierarchy and that some individuals can find certain higher-order needs more important than lowerlevel ones (1943). According to Spector (2003), many individuals in Western society have the first four needs met and may never have experienced deprivations of one or more of them, especially food.

Over the years, the job market has fluctuated greatly in both directions. During these times of job market insecurity; numerous motivating factors have influenced various members of the population to keep their existing jobs. In 2011, the Bureau of Labor Statistics reported 139.3 million people in the United States were employed. Of that number, 113 million people were employed full-time while 26.3 million were employed part-time (Berger, 2011). A study conducted by World Bank from 2009 to 2013, found that two percent of the total number employed in the United States were employed in agriculture.

Agriculture is an essential part of the United States. William Jennings Bryan (Bryan, n.d.) once said, "Burn down your cities and leave our farms, and your cities will spring up again as if by magic; but destroy our farms and the grass will grow in the streets of every city in the country," purporting agriculture to keep cities alive and 
flourishing. Even in the early stages of our nation, President George Washington understood the importance of agriculture. "It will not be doubted that with reference either to individual or national welfare, agriculture is of primary importance" (G. Washington, 1793). Many researchers have proven these statements true over time. Martin, Milne-Home, Barrett, Spalding, and Jones (2000) found a need for higher education to prepare graduates for the demands of the industry. The National Research Council (1996) stated that the modern United States food and agriculture system is large, complex, diverse, and dynamic, and colleges of agriculture should reflect these contemporary changes. A study by Goecker, Whatley, and Gilmore (1999) states that much greater efforts will be required to attract sufficient numbers of outstanding students to prepare for very challenging careers in the world's food, agricultural and natural resources system. Goecker, Smith, Smith, \& Goetz (2010) estimate that, the agricultural, food, and renewable natural resources sectors of the United States economy will generate an estimated 54,400 annual openings for individuals with baccalaureate or higher degrees in food, renewable energy, and environmental specialties between 2010 and 2015.

\section{Problem Statement}

The current world population is approximately seven billion, a number that is constantly on the rise (USCB, 2014). As the world population increases the need to feed, clothe, and house more people will require more skilled laborer in the agricultural sector. Agriculture will need a work force of dedicated and highly motivated individuals to move the industry forward. In order to attract innovative and hardworking people to engage in careers in the agricultural sector, we need to understand the motivation factors that impact those decisions. By studying students currently enrolled in a college offering 
agricultural related degrees will help us to understand the factors that impacted their decisions to pursue agriculturally related programs of study.

\section{Purpose of the Study}

The purpose of this study is to determine the motivational factors contributing to an undergraduate student's choice to pursue an agriculturally related career. By determining factors which motivate students to choose agriculturally related careers, high school teachers, guidance counselors, extension agents, and colleges have a better understanding as to how to advise and recruit students to pursue agriculturally related careers.

\section{Objective of the Study}

The objective of the study was to determine the motivational factors associated with career choices of undergraduate students pursuing degrees in agriculturally related fields. The following research questions were used to guide this study:

1. What factors influenced students to pursue a career in an agriculturally related field?

2. Do students enrolled in an agricultural degree program anticipate pursuing a career in an agriculturally related field?

3. Which youth organizations have agricultural degree students participated in prior to college?

4. Is there an association between previous experience working on a farm or ranch and pursuing an agriculturally related career?

5. Is there an association between previous experience working in an agricultural business and pursuing an agriculturally related career? 
6. Is there an association between parents or relatives working in an agricultural business and pursuing an agriculturally related career?

7. Is there an association between parents or relatives working on a farm or ranch and pursuing an agriculturally related career?

8. Is there an association between gender and pursuing an agriculturally related career?

9. Is there an association between enrollment in high school agricultural courses and pursuing an agriculturally related career?

10. Is there an association between college agricultural related organization involvement courses and pursuing an agriculturally related career?

\section{Limitations of the Study}

This study was limited to undergraduate students enrolled in the Davis College of Agriculture, Natural Resources and Design at West Virginia University during spring semester of 2014. The areas of study included: Agribusiness Management and Rural Development, Agriculture and Extension Education, Agroecology, Agronomy, Animal and Nutritional Science, Applied and Environmental Microbiology, Biochemistry, Design Studies, Environmental and Natural Resource Economics, Environmental Protection, Fashion Design and Merchandising, Forest Resource Management, Horticulture, Human Nutrition and Foods, Interior Design, Landscape Architecture, Multidisciplinary Studies, Recreation, Parks and Tourism Resources, Soil Sciences, Wildlife and Fisheries Resources, and Wood Science and Technology. 


\section{CHAPTER II}

\section{Review of Literature}

People begin looking towards career choices at a very young age and may choose their jobs based on a single motivational factor or multiple factors. Maslow's (1943) need hierarchy theory states that the fulfillment of human needs is necessary for both physical and psychological health. Maslow (1943) states that human needs arrange themselves in hierarchies of importance, which implies that a prior need has been fulfilled first and that every need is dependent on the satisfaction or dissatisfaction of another need. Maslow's five levels in his need hierarchy build from the lowest level of Physiological, through Safety, Love, Esteem, and Self-actualization (Spector, 2003). "According to Maslow, a need must be unmet to be motivating, and people are motivated by the lowest-level need that is unmet" (Spector, 2003, p. 87). Herzberg (1976) developed a theory on Motivational-Hygiene which is similar to Maslow's Needs hierarchy. The first basic biological need serves as the necessities to human survival. People work for money to buy food. Food serves as the common motivating factor; however, once satisfied physically, making money becomes the primary need. The other need described by Herzberg is the desire to be successful, experience achievement, and grow psychologically (Garton \& Robinson, 2006). Maslow understood, however, that exceptions could occur. Many individuals in Western society have the first four needs met and may never experience deprivations of one or more of them, especially food. Therefore, the basic needs are not motivating for those individuals (Spector, 2003).

Previous studies indicate that there are a number of motivational factors which impact career decisions and pathways, those may include: money; such as a focus on 
income or being able to pay the bills (Buckingham \& Clifton, 2001; Ng, 2008; Pink, 2009; Pink, 2008; Rawls, Martin, Negatu, \& Robertson, 1994). Interest in the work field was determined to be a contributing factor (Associated Press, 2010; Ng, 2008; Pink, 2008; Reis \& Kahler, 1997); as well as fulfilling a purpose (Buckingham \& Clifton, 2001; Llopis, 2012; Pink, 2009;), career advancement opportunities (Adams, 2012; Llopis, 2012; Ng, 2008), autonomy (Llopis, 2012; Pink, 2009), and job stability (Llopis, 2012; Ng, 2008). Each of these factors contributes to choosing a career. Several studies have reported that parents, family, teachers, counselors, and/or friends rank high as motivating factors when deciding on career options (Bowen \& Lee, 1984; Donnermeyer and Kreps, 1994; Cole, 1985; Faulks, 1969; Findlay \& Rawls, 1984; Miller, Williams, \& Sprouse, 1984; Reis \& Kahler, 1997; Sutpin \& Newsom-Stewart, 1995; Wildman \& Torres, 2001;). Rawls, Martin, Negatu, \& Robertson (1994) found that considerations can include the nature of the work (i.e., working outdoors, working with people and/or animals), availability and location of the job, and prestige of career area.

The Bureau of Labor Statistics reported that the number of working civilians numbers 238.7 million people in the United States alone; 113 million people are employed full-time and 26.3 million people are employed part-time (Berger, 2011). Of those 238.7 million people, a wide range of motivational factors contribute to those employment choices. Whether it is money, purpose, interest, or availability, etc., each employee has individual characteristics motivating them to work at their current position.

Several studies have shown evidence of a need for higher education to prepare graduates for the demands of the industry. The field of agriculture, which is a large, complex, diverse and dynamic industry, will need well versed individuals to take on 
contemporary changes (Martin, Milne-Home, Barrett, Spalding \& Jones, 2000; National Research Council, 1996; Scanlon, Bruening \& Cordero, 1996; Thomson, 1994). Students usually equate a career in agriculture with farming or ranching only, rather than with the science or business side of agriculture (Orthel, Sorensen, Lierman \& Riesenburg, 1989). Fishbein and Ajzen (1975) determined that intentions to participate in an activity could be predicted based on a person's knowledge, observations, or other information held about some issue or event. Greenwald (1989) found very similar results in that individuals with positive attitudes toward a subject or situation tended to evaluate them positively. A study conducted by Krueger and Riesenburg (1991) found that secondary students perceived an agricultural career to be boring, hard work with poor pay, and involving more muscle than brain. Participants perceived an agricultural career as an outdoor job that involves more men than women. Goecker, Smith, Smith, \& Goetz (2010) reported that about 53, 500 graduates will enter the job market each year, with about half of the total graduates, 29,300 expected to earn degrees in agriculturally related fields with degrees from colleges of agriculture and life sciences, forestry and natural resources, and veterinary medicine.

Goecker, et al., (2010) reported that agricultural, food, and renewable natural resources sectors of the U.S. economy will generate an estimated 54,400 annual openings for individuals with baccalaureate or higher degrees in food, renewable energy, and environmental specialties between 2010 and 2015 . They project that $74 \%$ of the jobs will be in business and science occupations; $15 \%$ in agriculture and forestry production; and $11 \%$ in education, communication, and governmental services" (Goecker, et al., 2010). 
Goecker, et al., (2010) found that employers prefer graduates from colleges of agriculture and life sciences, forestry and natural resources, and veterinary medicine who tend to have relatively stronger interests and more extensive work experiences for careers in food, renewable energy, and the environment than those from allied fields of study. Several studies including those by Martin, et al., (2000); National Research Council (1996); Thomson (1994); Scanlon, et al., (1996) have shown evidence of a need for higher education to prepare graduates for the demands of the agricultural industry.

The U.S. Department of Labor (2009) projects significant growth in selected food, renewable energy, and environment jobs during 2008-18. There will be a need during the span of $2008-2018$ for skilled workers in diverse areas which will include agricultural inspectors, animal scientists, environmental engineers, environmental scientists and specialists, including health, food scientists and technologists, hydrologists, management analysts, market research analysts, natural sciences managers, pest Control workers, public relations specialists, recreation workers, sales managers, soil and plant scientists, and veterinarians (Goecker, et al., 2010).

Numerous studies have shown that the agricultural industry will have a continued need for skilled graduates to pursue employment in agriculturally related fields. With so many different types of positions available in the U.S., many people will be needed to fill the positions which require baccalaureate or higher degrees in food, renewable energy, and environmental specialties between 2010 and 2015 (Goecker, et al, 2010).

Examining the factors which motivate students to seek careers in agriculture may help, to recruit and train individuals who would purse agricultural employment opportunities and provide a skilled workforce to meet the demands. 


\section{CHAPTER III}

\section{Methodology}

\section{Purpose of the Study}

The purpose of this study was to determine the motivational factors contributing to an undergraduate student's choice to pursue an agriculturally related career. By determining factors which motivate students to choose agriculturally related careers, high school teachers, guidance counselors, extension agents, and colleges have a better understanding as to how to advise and recruit students to pursue agriculturally related careers.

\section{Objective of the Study}

The objective of the study was to determine the motivational factors associated with career choices of undergraduate students pursuing degrees in agriculturally related fields. The following research questions were used to guide this study:

1. What factors influenced students to pursue a career in an agriculturally related field?

2. Do students enrolled in an agricultural degree program anticipate pursuing a career in an agriculturally related field?

3. Which youth organizations have agricultural degree students participated in prior to college?

4. Is there an association between previous experience working on a farm or ranch and pursuing an agriculturally related career?

5. Is there an association between previous experience working in an agricultural business and pursuing an agriculturally related career? 
6. Is there an association between parents or relatives working in an agricultural business and pursuing an agriculturally related career?

7. Is there an association between parents or relatives working on a farm or ranch and pursuing an agriculturally related career?

8. Is there an association between gender and pursuing an agriculturally related career?

9. Is there an association between enrollment in high school agricultural courses and pursuing an agriculturally related career?

10. Is there an association between college agricultural related organization involvement courses and pursuing an agriculturally related career?

\section{Research Design}

This study utilized a descriptive research design using an online questionnaire. According to Ary, Jacobs, Sorensen \& Walker (2010) descriptive research allows you to ask questions about certain factors and describing the situation without manipulating the variables. This survey research was nonexperimental where questions were asked about peoples' beliefs, opinions, and behavior (Ary, Jacobs, Sorensen \& Walker, 2010).

\section{Population}

The target population was all undergraduate students enrolled in the Davis College of Agriculture, Natural Resources and Design at West Virginia University. The Davis College of Agriculture, Natural Resource and Design offers twenty-two undergraduate agriculturally related majors. The accessible population consisted of all undergraduate students enrolled in the Davis College of Agriculture, Natural Resources and Design at West Virginia University during spring semester of $2014(\mathrm{~N}=1,673)$. 


\section{Instrumentation}

The questionnaire was developed by the researcher. It consisted of Likert type questions and included sections on family influence, self-motivators, use of skills, career opportunities, and experiences as well as demographic questions. Demographic questions included parent/guardian occupations, anticipated starting salaries in chosen field, where the participants resided as well as the types of organizations to which they belong. The instrument was presented to a panel of faculty members in Agricultural and Extension Education and Extension Specialists at West Virginia University to establish content and face validity. Each individual on the panel had extensive teaching and research experience. The faculty panel determined that the instrument had face and content validity. Reliability was determined using SPSS. Cronbach's alpha was calculated for each of the sections. The reliability of sections family influence, selfmotivations, experiences, use of skills, and career opportunities were found to be exemplary at $.916, .957, .916, .969$, and .951 , respectively (Robinson, Shaver, \& Wrightsman, 1991). The instrument was established as a reliable measure.

\section{Data Collection Procedures}

The survey was conducted using an online survey administered through Surveymonkey.com. An internal data request was made through the University Registrar's office to receive the email addresses for all undergraduate students in the Davis College of Agriculture, Natural Resources and Design for spring semester 2014. Once the email addresses were obtained a dedicated college study listserv was set up through the Davis College Office of Information Technology Network Manager. The listserv contained the e-mail addresses for all undergraduates $(\mathrm{N}=1673)$. An email 
message explaining the study, with a request to participate in the study and a link to the online survey was sent to all 1673 students email addresses. Responses to the survey were anonymous therefore the survey was sent to all students a total of four times, one week apart. The survey was open for a period of one month from April 1 to May 1. Data were collected and analyzed using Statistical Package for the Social Science (SPSS).

\section{Analysis of Data}

The data were downloaded from SurveyMonkey into an Excel spreadsheet and were analyzed through the Statistical Package for the Social Sciences (SPSS). The level of significance was set $a$ priori at $\alpha \leq .05$ for all statistical tests. Frequencies, means, standard deviations, t-tests, and chi-squares were run.

T-tests were run in association with the non-response error. One hundred and fifty-two students $(50.2 \%)$ were late respondents. One hundred and fifty-one students $(49.8 \%)$ were early respondents. Due to all five sections having a significant difference in the early-late responses, generalizations were limited to the 303 participants of the study.

\section{Use of Findings}

After careful analysis of the survey data, the findings will be used to determine which factors motivate individuals to seek agriculturally related careers. This information has the potential to be used for youth development organizations in determining educational programs best relating to possible career choices as well as college departments for recruiting purposes. This information will also be useful for agricultural business owners to better understand the motivation behind their employees and future employees' career paths. 


\section{CHAPTER IV}

\section{Findings}

\section{Purpose of the Study}

The purpose of this study was to determine the motivational factors contributing to an undergraduate student's choice to pursue an agriculturally related career. By determining factors which motivate students to choose agriculturally related careers, high school teachers, guidance counselors, extension agents, and colleges have a better understanding as to how to advise and recruit students to pursue agriculturally related careers.

\section{Objective of the Study}

The objective of the study was to determine the motivational factors associated with career choices of undergraduate students pursuing degrees in agriculturally related fields. The following research questions were used to guide this study:

1. What factors influenced students to pursue a career in an agriculturally related field?

2. Do students enrolled in an agricultural degree program anticipate pursuing a career in an agriculturally related field?

3. Which youth organizations have agricultural degree students participated in prior to college?

4. Is there an association between previous experience working on a farm or ranch and pursuing an agriculturally related career?

5. Is there an association between previous experience working in an agricultural business and pursuing an agriculturally related career? 
6. Is there an association between parents or relatives working in an agricultural business and pursuing an agriculturally related career?

7. Is there an association between parents or relatives working on a farm or ranch and pursuing an agriculturally related career?

8. Is there an association between gender and pursuing an agriculturally related career?

9. Is there an association between enrollment in high school agricultural courses and pursuing an agriculturally related career?

10. Is there an association between college agricultural related organization involvement courses and pursuing an agriculturally related career?

\section{Demographic Characteristics}

The accessible population for this study consisted of all undergraduates in the Davis College of Agriculture, Natural Resources and Design at West Virginia University $(\mathrm{N}=1673)$. A census of all 1,673 undergraduates was conducted, 303 surveys were returned for a response rate of 18 percent.

Seventy of the participants (36.65\%) were male while 121 participants $(63.35 \%)$ were female. Of the 303 participants, 180 (93.75\%) were white, five (2.60\%) were black or African American, one participant (.52\%) was Asian, and six students (3.13\%) were 'other.' Five of the participants (2.67\%) were Hispanic or Latino while 182 students (97.33\%) were Non-Hispanic or Latino (see Table 1). 
Table 1

Gender, Race and Ethnicity

\begin{tabular}{|c|c|c|}
\hline & $\mathrm{N}$ & $\%$ \\
\hline \multicolumn{3}{|l|}{ Gender } \\
\hline Male & 70 & 36.65 \\
\hline Female & 121 & 63.35 \\
\hline Total & 191 & 100.00 \\
\hline \multicolumn{3}{|l|}{ Race } \\
\hline American Indian or Alaskan Native & 0 & .00 \\
\hline Asian & 1 & .52 \\
\hline Black or African American & 5 & 2.60 \\
\hline Native Hawaiian or Pacific Islander & 0 & .00 \\
\hline White & 180 & 93.75 \\
\hline Other & 6 & 3.13 \\
\hline Total & 192 & 100.00 \\
\hline \multicolumn{3}{|l|}{ Ethnicity } \\
\hline Hispanic or Latino & 5 & 2.67 \\
\hline Non-Hispanic or Latino & 182 & 97.33 \\
\hline Total & 187 & 100.00 \\
\hline
\end{tabular}

One hundred and ninety-three students reported their age for the survey. Seventyone participants $(36.79 \%)$ were between the ages of 18 and 20 . Twenty-one to 23 year olds comprised 87 participants $(45.08 \%)$. Sixteen students $(8.29 \%)$ were between the ages of 24 and 26 . Nine students (4.66\%) were between the ages of 27 and 29 years of age. Ten of the participants (5.18\%) were 30 years old or above (see Table 2 ). 
Table 2

Age Range and Home State

\begin{tabular}{|c|c|c|}
\hline & $\mathrm{N}$ & $\%$ \\
\hline \multicolumn{3}{|l|}{ Age Range } \\
\hline 18 to 20 & 71 & 36.79 \\
\hline 21 to 23 & 87 & 45.08 \\
\hline 24 to 26 & 16 & 8.29 \\
\hline 27 to 29 & 9 & 4.66 \\
\hline 30 and up & 10 & 5.18 \\
\hline Total & 193 & 100.00 \\
\hline \multicolumn{3}{|l|}{ Home State } \\
\hline West Virginia & 112 & 58.33 \\
\hline Pennsylvania & 19 & 9.90 \\
\hline Maryland & 18 & 9.38 \\
\hline Ohio & 18 & 9.38 \\
\hline Virginia & 10 & 5.21 \\
\hline New Jersey & 4 & 2.08 \\
\hline Arizona & 2 & 1.04 \\
\hline Colorado & 1 & 0.52 \\
\hline Delaware & 1 & 0.52 \\
\hline Massachusetts & 1 & 0.52 \\
\hline Nevada & 1 & 0.52 \\
\hline New Hampshire & 1 & 0.52 \\
\hline North Carolina & 1 & 0.52 \\
\hline Oklahoma & 1 & 0.52 \\
\hline Texas & 1 & 0.52 \\
\hline Puerto Rico & 1 & 0.52 \\
\hline Total & 192 & 100.00 \\
\hline
\end{tabular}


Sixteen states were represented by participants. One-hundred twelve participants (58.33\%) were from the state of West Virginia. Pennsylvania was represented by 19 students (9.90\%). Eighteen students (9.38\%) reported they were from Maryland and Ohio. Ten participants (5.21\%) were from Virginia. Four students (2.08\%) represented the state of New Jersey. Two students (1.04\%) were from Arizona. Colorado, Delaware, Massachusetts, Nevada, New Hampshire, North Carolina, Oklahoma, Texas, and Puerto Rico each had one student $(0.52 \%)$ representing the state (see Table 2 ).

One hundred and ninety-two participants gave their place of residency. Fifty-two participants (27.08\%) came from a small farm or ranch (see Table 3). Thirty-four students (17.71\%) were from a rural area, but not a farm or ranch. Students who were from small towns, less than 5,000 people, included 26 participants (13.54\%). Fifty-five students $(28.65 \%)$ were from a small city or suburb with a population of 5,000 to 50,000 people. Twenty-five students (13.02\%) were from an urban area or city with a population of more than 50,000 people (see Table 3).

Of the 303 participants, 18 of the students $(9.28 \%)$ were classified as freshman. Thirty-three students (17.01\%) were sophomores. There were 43 participants who were junior status, and 95 students (48.97\%) were seniors. Four participants (2.06\%) classified themselves as graduate students. One student (.52\%) was a non-degree seeking participant (see Table 3).

Respondents were asked to report their grade point average by ranges. There were four participants $(2.06 \%)$ who preferred not to respond to this question. Sixty-six students (34.02\%) indicated their grade point average was 3.5 to 4.0 . Seventy-two students $(37.11 \%)$ reported a grade point average between 3.0 and 3.49. There were 49 
participants (25.26\%) who had a GPA between 2.5 and 2.9. Three respondents (1.55\%)

had a GPA below 2.49 (see Table 3).

Table 3

Place of Residency, Student Status, Grade Point Average

\begin{tabular}{lcc}
\hline & $\mathrm{N}$ & $\%$ \\
\hline Place of Residency & & \\
$\quad$ Small Farm or Ranch & 52 & 27.08 \\
$\quad$ Rural Area, but not on a & 34 & 17.71 \\
$\quad$ Farm or Ranch & & 13.54 \\
Small Town; <5,000 & 26 & 28.65 \\
Small City or Suburb; & 55 & 13.02 \\
5,000 to 50,000 & & \\
Urban Area, City; $>$ & 25 & 100.0 \\
50,000 & 192 & \\
Total & & 9.28 \\
Student Status & 18 & 17.01 \\
Freshman & 33 & 22.16 \\
Sophomore & 43 & 48.97 \\
Junior & 95 & 2.06 \\
Senior & 4 & .52 \\
Graduate Student & 194 & \\
Non-Degree seeking & & \\
student & & \\
Total & & \\
\hline
\end{tabular}


Table 3 (continued)

Place of Residency, Student Status, Grade Point Average

\begin{tabular}{lcc}
\hline & $\mathrm{N}$ & $\%$ \\
\hline Grade Point Average & 66 & 34.02 \\
$3.5-4.0$ & 72 & 37.11 \\
$3.0-3.49$ & 49 & 25.26 \\
$2.5-2.9$ & 3 & 1.55 \\
Below 2.49 & 4 & 2.06 \\
Prefer not to respond & 194 & 100.00 \\
Total & & \\
\hline
\end{tabular}

Students were asked to mark the organizations they participated in as a child. Sixty-three persons (20.79\%) were involved in 4-H, and 61 students $(20.13 \%)$ were involved in FFA (see Table 4). Twenty-seven people (8.91\%) took part in Boy Scouts as a youth while 49 students (16.17\%) were involved in Girl Scouts. Seven participants $(2.31 \%)$ reported involvement in a junior breed association. Twenty individuals $(6.60 \%)$ were involved in the Farm Bureau. One participant (.33\%) took part in Grange. Eight individuals (2.5\%) marked 'other' organizations (see appendices D). 
Table 4

Participation in Organizations as a Youth

\begin{tabular}{lcc} 
& N & $\%$ \\
\hline $4-H$ & 63 & 20.79 \\
FFA & 61 & 20.13 \\
Boy Scouts & 27 & 8.91 \\
Girl Scouts & 49 & 16.17 \\
Junior Breed Association & 7 & 2.31 \\
Farm Bureau & 20 & 6.60 \\
Grange & 1 & .33 \\
Other & 8 & 2.5 \\
\hline
\end{tabular}

Participants were asked to give their focus of study while at West Virginia University. Agribusiness Management and Rural Development was being studied by 11 participants (5.91\%). Environmental Protection was the focus of five participants $(2.69 \%)$ (see Table 5). One student (0.54\%) was studying Fashion Design and Merchandising. Fourteen participants (7.53\%) were studying Forest Resource Management. Horticulture was being studied by eight of the participants $(4.30 \%)$. Thirteen students $(6.99 \%)$ were focused on Human Nutrition and Foods. Three participants (1.61\%) were studying Interior Design. Landscape Architecture was being studied by ten individuals (5.38\%). Recreation, Parks and Tourism Resources had nine participants $(4.83 \%)$ focused on the program. Soil Science was being studied by one participant (0.54\%). Agriculture and Extension Education was being studied by 31 
individuals (16.67\%). Wildlife and Fisheries Resources was being studied by sixteen participants (8.60\%). Wood Science and Technology was focused on by three participants (1.61\%). Agroecology was studied by five participants (2.69\%). Animal and Nutritional Science was being studied by 41 participants (22.04\%). Applied and Environmental Microbiology was being studied by one individual ( $0.54 \%)$. Biochemistry was being studied by nine participants (4.83\%). Design Studies was focused on by three participants (1.61\%). Environmental and Natural Resource Economics was being studied by two participants (1.08\%). Seventeen participants (9.14\%) marked 'other' due to double majors and extra minors (see Appendices E).

Table 5

Study Focus at West Virginia University

\begin{tabular}{lcc}
\hline & $\mathrm{N}$ & $\%$ \\
\hline Agribusiness Management and Rural & 11 & 5.91 \\
Development & 5 & 2.69 \\
Environmental Protection & 1 & 0.54 \\
Fashion Design and Merchandising & 14 & 7.53 \\
Forest Resource Management & 8 & 4.30 \\
Horticulture & 13 & 6.99 \\
Human Nutrition and Foods & 3 & 1.61 \\
Interior Designs & 10 & 5.38 \\
Landscape Architecture & 9 & 4.83 \\
Recreation, Parks and Tourism Resources & 1 & 0.54 \\
Soil Sciences & 31 & 16.67 \\
Agriculture and Extension Education & & \\
\hline
\end{tabular}


Table 5 (continued)

Study Focus at West Virginia University

\begin{tabular}{lcc}
\hline & $\mathrm{N}$ & $\%$ \\
\hline Wildlife and Fisheries Resources & 16 & 8.60 \\
Wood Science and Technology & 3 & 1.61 \\
Agroecology & 5 & 2.69 \\
Animal and Nutritional Science & 41 & 22.04 \\
Applied and Environmental Microbiology & 1 & 0.54 \\
Biochemistry & 9 & 4.83 \\
Design Studies & 3 & 1.61 \\
Environmental and Natural Resource & & 1.08 \\
Economics & 2 & 9.14 \\
Other & 17 & 100.0 \\
Total & 186 & \\
\hline
\end{tabular}

Two hundred and ninety-nine students responded to the question as to whether or not they anticipated pursuing an agriculturally related career. Of the 299 respondents, 201 students (67.2\%) answered yes while 98 students $(32.8 \%)$ responded no (see Table $6)$.

One hundred and ninety-four participants answered a questions pertaining to how satisfied they were with their current career choice. One hundred and sixteen participants $(59.79 \%)$ were very satisfied with their career choice while sixty-four students $(32.99 \%)$ were satisfied with their career choice. Four individuals $(2.06 \%)$ were dissatisfied with their career choices. Two students $(1.03 \%)$ were very dissatisfied with their career 
choice. Eight students $(4.12 \%)$ were uncertain about the career choice they had made (see Table 6).

Table 6

Pursuing Agriculturally Related Careers and Satisfaction with Career Choice

\begin{tabular}{lcc}
\hline & $\mathrm{N}$ & $\%$ \\
\hline Pursuing Agriculturally Related Careers & 201 & 67.2 \\
Yes & 98 & 32.8 \\
No & 299 & 100.00 \\
Total & & \\
Satisfaction with Career Choice & 116 & 59.79 \\
Very Satisfied & 64 & 32.99 \\
Satisfied & 4 & 2.06 \\
Dissatisfied & 2 & 1.03 \\
Very Dissatisfied & 8 & 4.12 \\
Uncertain & 194 & 100.0 \\
Total & &
\end{tabular}

When asked what the estimated starting salary of their intended career would be, seven students (3.65\%) estimated their starting salary to be under $\$ 20,000$ (see Table 7). Sixty-nine participants (35.94\%) estimated their starting salary to be $\$ 20,001-\$ 40,000$. Seventy-one students $(36.98 \%)$ estimated $\$ 40,001-\$ 60,000$ as their starting salary. Thirty-three participants (17.19\%) estimated their starting salary to be $\$ 60,001-$ $\$ 80,000$. Eight students $(4.17 \%)$ estimated $\$ 80,001-\$ 100,000$ as their starting salary, 
while four students $(2.08 \%)$ estimated $\$ 100,001$ and above for their starting salary (see Table 7).

Table 7

Estimated Starting Salary of Career Choice

\begin{tabular}{lcc}
\hline & $\mathrm{N}$ & $\%$ \\
\hline Under $\$ 20,000$ & 7 & 3.65 \\
$\$ 20,001-40,000$ & 69 & 35.94 \\
$40,001-60,000$ & 71 & 36.98 \\
$\$ 60,001-80,000$ & 33 & 17.19 \\
$\$ 80,001-100,000$ & 8 & 4.17 \\
$\$ 100,001$ and above & 4 & 2.08 \\
Total & 192 & 100.00 \\
\hline
\end{tabular}

Respondents were asked to indicate the areas that best describe their prior agricultural background. Sixty students $(19.80 \%)$ reported they had a background in forestry-natural resources (see Table 8). Ninety-four participants $(31.02 \%)$ had a background in animal science. Fifty-four students (17.82\%) reported a background in plant and soil science and/or horticulture. Forty-one students (13.53\%) reported a background in agribusiness. Forty-eight students (15.84\%) claimed to have no agricultural background (see Table 8) (see Appendix C).

The students were also asked to respond to a series of yes or no questions in reference to their life experiences. Sixty-three students $(32.31 \%)$ reported being first generation college students (see Table 8). One hundred and fifteen students (58.97\%) had previous experience on a farm or ranch. Sixty-nine students $(35.94 \%)$ had 
experience in an agricultural business. One hundred and twenty-two students $(62.89 \%)$ have relatives that worked on a farm or ranch. Seventy-seven students (40.10\%) have relatives that worked in an agricultural business. Seventy-six participants (38.97\%) took high school agricultural courses. Ninety-six students (49.48\%) went to a high school that offered courses in agricultural education. Eighty-seven students (45.08\%) were involved in agriculturally related organizations at the college level (see Table 8).

Table 8

Type of Agricultural Background and Experiences

$\mathrm{N} \quad \%$

Agricultural Backgrounds:

Forestry-Natural Resources $\quad 60$

19.80

Animal Science $\quad 94$

31.02

Plant and Soil Science Horticulture

54

17.82

Agribusiness

41

No Agricultural Background

48

15.84

Experiences:

First Generation College Student 63

Worked on a Farm or Ranch

115

58.97

Worked in an Agricultural Business

69

Relatives Working on a Farm-Ranch

122

62.89

Relatives Working in an Agricultural Business

77

40.10

High School Agricultural Courses

76

38.97

High School Offer Courses in Agricultural

Education

College Agriculturally Related Organization Involvement 
Students were asked to respond to the open-ended question, "What is your mother/ female guardian's occupation?" Due to the variation of responses among participants, all answers were categorized into general word titles that best fit the respondent's answers. Eleven students (5.8\%) indicated their mother or female guardian worked in an administrative position (see Table 9). Five of the mothers/ female guardians (2.6\%) worked in an agricultural position. Twenty-three students (12.1\%) indicated their mothers/female guardian was in a business position. Three mothers/female guardians (1.6\%) were cosmetologists. Three of the mothers/female guardians $(1.6 \%)$ were deceased while three other female parental figures $(1.6 \%)$ were disabled. One mothers/female guardian $(0.5 \%)$ was in a driver position. Thirty-nine students indicated their mother or female guardian (20.5\%) were in educational positions. One of the mothers/female guardians $(0.5 \%)$ was an engineer. Eleven students $(5.8 \%)$ indicated that their mothers/female guardian worked in financing. Six mothers/ female guardians (3.2\%) work in government positions. Twenty-three students (12.1\%) indicated their mother figure was a homemaker. Twelve mothers/female guardians (6.3\%) were laborers. Two mothers/female guardians were working as lawyers. Five mothers/female guardians held management positions in the work place. Twenty-four students (12.6\%) indicated their mothers/female guardian worked in the medical field, while one individual's mother $(0.5 \%)$ worked in the ministry field. Seven students (3.7\%) indicated their mothers/female figure were scientists. Six mothers/female guardians $(3.2 \%)$ were unemployed and four students $(2.1 \%)$ indicated the question was inapplicable to their current situation (see Table 9) (see Appendix A). 
Table 9

Mother/ Female Guardian Occupations

\begin{tabular}{|c|c|c|}
\hline & $\mathrm{N}$ & $\%$ \\
\hline Administrative & 11 & 5.8 \\
\hline Agriculture & 5 & 2.6 \\
\hline Business & 23 & 12.1 \\
\hline Cosmetology & 3 & 1.6 \\
\hline Deceased & 3 & 1.6 \\
\hline Disabled & 3 & 1.6 \\
\hline Driver & 1 & 0.5 \\
\hline Education & 39 & 20.5 \\
\hline Engineer & 1 & 0.5 \\
\hline Finances & 11 & 5.8 \\
\hline Government & 6 & 3.2 \\
\hline Homemaker & 23 & 12.1 \\
\hline Laborer & 12 & 6.3 \\
\hline Law & 2 & 1.1 \\
\hline Management & 5 & 2.6 \\
\hline Medical & 24 & 12.6 \\
\hline Ministry & 1 & 0.5 \\
\hline Not Applicable & 4 & 2.1 \\
\hline Scientist & 7 & 3.7 \\
\hline Unemployed & 6 & 3.2 \\
\hline Total & 190 & 100.0 \\
\hline
\end{tabular}


Students were asked to respond to the open-ended question, "What is your father/ male guardian's occupation?" Due to the response variation among participants, all answers have been categorized into general word titles that best fit the respondent's answer. Eleven students (5.9\%) indicated their father or male guardian is in an agricultural position (see Table 10). Ten students (5.4\%) indicated their father or male figure was involved in business. Eleven fathers or male guardians (5.9\%) have construction positions. Three students (1.6\%) indicated the father or male guardian is deceased. Seven fathers or male guardians (3.8\%) hold driver positions. Twelve students (6.5\%) indicated their father figure held an educational position. Two father figures (1.1\%) were electricians, while twelve students (6.5\%) indicated their father held an engineering position. Four participants (2.2\%) indicated their father figure was in finances. Fifteen of the father figures (8.1\%) held government jobs. Twenty-four of the father figures (13.0\%) held general laborer positions. Thirteen students (7.0\%) indicated their father figure held a position based in law enforcement or as a lawyer. Twenty students (10.8\%) indicated their father figure held management positions. Twelve students $(6.5 \%)$ indicated the father figure of the house was a mechanic. Nine father figures $(4.9 \%)$ hold medical positions. Two father figures $(1.1 \%)$ held military positions and three (1.6\%) held mining positions. Seven students (3.8\%) indicated their father or male guardian was in the ministry. One student $(0.5 \%)$ indicated his or her father figure had retired. Five father figures $(2.7 \%)$ were scientists and two (1.1\%) of the father figures were unemployed. 
Table 10

Father/ Male Guardians Occupation

\begin{tabular}{|c|c|c|}
\hline & $\mathrm{N}$ & $\%$ \\
\hline Agriculture & 11 & 5.9 \\
\hline Business & 10 & 5.4 \\
\hline Construction & 11 & 5.9 \\
\hline Deceased & 3 & 1.6 \\
\hline Driver & 7 & 3.8 \\
\hline Education & 12 & 6.5 \\
\hline Electrician & 2 & 1.1 \\
\hline Engineer & 12 & 6.5 \\
\hline Finances & 4 & 2.2 \\
\hline Government & 15 & 8.1 \\
\hline Laborer & 24 & 13.0 \\
\hline Law & 13 & 7.0 \\
\hline Management & 20 & 10.8 \\
\hline Mechanical & 12 & 6.5 \\
\hline Medical & 9 & 4.9 \\
\hline Military & 2 & 1.1 \\
\hline Miner & 3 & 1.6 \\
\hline Ministry & 7 & 3.8 \\
\hline Retired & 1 & 0.5 \\
\hline Scientist & 5 & 2.7 \\
\hline Unemployed & 2 & 1.1 \\
\hline Total & 185 & 100.0 \\
\hline
\end{tabular}


Students were given a series of Likert scale items to determine which traits or experiences were most influential in their decision to pursue an agriculturally related career. The Likert items were broken into five constructs to better understand the influences of choosing agriculturally related careers. The first construct of 'Family Influence' consisted of 15 items, which included: "my parents influenced my decision," "teachers influenced my decision," "counselors influenced my decision," "my friends influenced my decision," "my 4-H leader(s) influenced my decision," "my agricultural teacher had an influence on my decision," " agriculture is a tradition in my family," "choosing an agriculturally related career is expected of me" "my family encouraged me to make the choice," "I wanted to do something my family has never done," "an agricultural professional influenced my choice," "Extension professional influenced my choice," "this decision was made to make my parents proud," "my parents insisted on an agriculturally related career," and "my significant others encouragement influenced my decision."

\section{Family Influence}

In regards to the statement, "my parents influenced my career decision," 92 students (34.85\%) marked not at all influential, 74 (28.03\%) believed parents were mildly influential, 57 (21.59\%) believed parents were moderately influential, while 37 (14.02\%) felt that parents were highly influential in their choice to work in an agriculturally related career. Four students (1.52\%) felt this was inapplicable to them (see Table 12).

In regard to "teachers influenced my career decision," 95 students (31.4\%) were not influenced, 62 students (20.5\%) were mildly influenced, 64 participants (21.1\%) were moderately influenced, and 38 students $(12.5 \%)$ were highly influenced by teachers. 
While five students $(1.7 \%)$ felt the question was not applicable to their situation (see Table 12).

In response to "counselors influenced my career decision," 152 students $(50.2 \%)$ reported they were not influenced, 56 students (18.5\%) were mildly influenced, 34 students $(11.2 \%)$ were moderately influenced, and 16 students $(5.3 \%)$ were highly influenced by their teacher. While seven students (2.3\%) felt this did not apply to their situation (see Table 12).

When asked whether "my friends influenced my career decision," 120 students $39.6 \%$ ) felt friends were not influential, 62 (20.5\%) reported friends were mildly influential, 53 students (17.5\%) were moderately influenced by friends, and 22 students (7.3\%) were highly influenced by their friends. While six students $(2.0 \%)$ felt this did not apply to their situation (see Table 12).

In response to "my 4-H leader(s) influenced my career decision," 135 students (44.6\%) felt 4-H leaders were not influential, 19 (6.3\%) were mildly influenced by 4-H leaders, $30(9.9 \%)$ were moderately influenced, and 15 students (5.0\%) were highly influenced by their 4-H leader (see Table 12). While 63 students (20.8\%) felt 4-H leader influence was not applicable to their situation.

In regard to the statement "my agricultural teacher had an influence on my decision," agricultural teachers did not influence 113 students (37.3\%), 25 students $(8.3 \%)$ were mildly influenced, 37 students (12.2\%) were moderately influenced, and 48 students (15.8\%) were highly influenced by their agricultural teacher (see Table 12). 
While 41 students (13.5\%) felt agricultural teachers influence was not applicable to their situation.

In response to "agriculture is a tradition in my family," 107 students $(35.3 \%)$ reported agriculture being a tradition in their family was not an influencing factor, 46 students (15.2\%) were mildly influenced by family tradition, 26 students $(8.6 \%)$ were moderately influenced, and 60 participants $(19.8 \%)$ were highly influenced by the fact that agriculture is a family tradition (see Table 12). While 23 participants $(7.6 \%)$ reported that family tradition in agriculture did not pertain to their situation.

In response to the statement, "choosing an agriculturally related career is expected of me," 175 students (57.8\%) indicated this was not an influencing factor, 43 students (14.2\%) were mildly influenced, 12 participants (4.0\%) were moderately influenced, and nine students (3.0\%) were highly influenced by the fact that they felt they were expected to choose an agriculturally related career (see Table 12). Twenty-four participants $(7.9 \%)$ reported this to be inapplicable to their situation.

In regard to the statement, "my family encouraged me to make the choice," 103 students (34.0\%) indicated this was not an influencing factor, while 53 students (17.5\%) were mildly influenced, 47 students (15.5\%) were moderately influenced, while 49 students $(16.2 \%)$ were highly influenced in their career because their family encouraged them to make the choice (see Table 12). Only 11 students (3.6\%) felt this was inapplicable to their situation.

In response to "I wanted to do something my family has never done," 104 individuals (34.3\%) indicated this was not an influencing factor, while 47 students 
(15.5\%) were mildly influenced, 32 participants (10.6\%) were moderately influenced, while 58 students (19.1\%) were highly influenced by the fact that they wanted to do something their family has never done (see Table 12$)$. Only 11 respondents (3.6\%) believed this did not pertain to their situation.

In regard to the statement "an agricultural professional influenced my choice," 110 individuals (36.3\%) indicated this was not an influencing factor, 31 students (10.2\%) were mildly influenced, 45 participants (14.9\%) were moderately influenced, while 52 students (17.2\%) were highly influenced by an agricultural professional (see Table 12). Twenty-two students (7.3\%) $\mathrm{r}$ this to be inapplicable to their situation.

In response to the statement "an Extension professional influenced my choice," 147 students (48.5\%) indicated this was not an influencing factor on career choice, while 43 students (14.2\%) reported an extension professional to be mildly influential, while an Extension professional was moderately influential and highly influential for 22 respondents (7.3\%) each (see Table 12). While 27 individuals (8.9\%) reported this factor was inapplicable to their situation.

When responding to the statement, "this decision was made to make my parents proud," 122 participants (40.3\%) indicated this was not an influencing factor, while 59 students (19.5\%) reported that to make their parents proud was mildly influential, 43 students (14.2\%) were moderately influenced, while 26 students (8.6\%) were highly influenced in their decision by wanting to make their parents proud of them (see Table 12). While 13 participants (4.3\%) reported this was inapplicable to their situation. 
In response to the statement, "my parents insisted on an agriculturally related career," 203 students $(67.0 \%)$ reported this had no influence on their decision, 26 students $(8.6 \%)$ were mildly influenced, seven students $(2.3 \%)$ were moderately influenced, and five participants (1.7\%) were highly influenced by their parents' insistence on an agriculturally related career (see Table 12). While 22 individuals (7.3\%) felt this was inapplicable to their situation.

In response to the statement that "my significant others encouragement influenced my decision," 182 students (60.1\%) reported this to have no influence on their situation while 20 students $(6.6 \%)$ were mildly influenced, 13 participants $(4.3 \%)$ were moderately influenced, and nine students (3.0\%) were highly influenced by their significant others encouragement (see Table 12). While 40 individuals (13.2\%) reported this did not apply to their situation.

\section{Constructs - Family Influences}

The Likert items were combined into composite constructs. The scale for the composite constructs is as follows: Not Influential $=1-1.5$, Mildly Influential $=1.6-$ 2.5, Moderately Influential $=2.6-3.5$, and Highly Influential $=3.6$ and above. Family Influences as a composite construct was rated as mildly influential $(\mathrm{M}=1.87, \mathrm{SD}=.58)$ (see Table 11 or Table 21).

Table 11

Construct- Family Influence

\begin{tabular}{lcccc}
\hline & $\mathrm{M}$ & $\mathrm{SD}$ & Min & Max \\
\hline Family Influence & 1.87 & .58 & 1.00 & 3.86 \\
\hline
\end{tabular}


Table 12

Family Influence

\begin{tabular}{|c|c|c|c|c|c|c|c|c|c|c|}
\hline & \multicolumn{2}{|c|}{$\begin{array}{l}\text { Not at All } \\
\text { Influential }\end{array}$} & \multicolumn{2}{|c|}{$\begin{array}{l}\text { Mildly } \\
\text { Influential }\end{array}$} & \multicolumn{2}{|c|}{$\begin{array}{l}\text { Moderately } \\
\text { Influential }\end{array}$} & \multicolumn{2}{|c|}{$\begin{array}{l}\text { Highly } \\
\text { Influential }\end{array}$} & \multicolumn{2}{|c|}{ Not Applicable } \\
\hline & $\mathrm{N}$ & $\%$ & $\mathrm{~N}$ & $\%$ & $\mathrm{~N}$ & $\%$ & $\mathrm{~N}$ & $\%$ & $\mathrm{~N}$ & $\%$ \\
\hline $\begin{array}{l}\text { My parents influenced my } \\
\text { decision }\end{array}$ & 92 & 34.85 & 74 & 28.03 & 57 & 21.59 & 37 & 14.02 & 4 & 1.52 \\
\hline Teachers influenced my decision & 95 & 35.98 & 62 & 23.48 & 64 & 24.24 & 38 & 14.39 & 5 & 1.89 \\
\hline $\begin{array}{l}\text { Councilors influenced my } \\
\text { decision }\end{array}$ & 152 & 57.36 & 56 & 21.13 & 34 & 12.83 & 16 & 6.04 & 7 & 2.64 \\
\hline $\begin{array}{l}\text { My friends influenced my } \\
\text { decision }\end{array}$ & 120 & 45.63 & 62 & 23.57 & 53 & 20.15 & 22 & 8.37 & 6 & 2.28 \\
\hline $\begin{array}{l}\text { My 4-H leader(s) influenced my } \\
\text { decision }\end{array}$ & 135 & 51.53 & 19 & 7.25 & 30 & 11.45 & 15 & 5.73 & 63 & 24.05 \\
\hline $\begin{array}{l}\text { My Agricultural teacher had an } \\
\text { influence on my decision }\end{array}$ & 113 & 42.80 & 25 & 9.47 & 37 & 14.02 & 48 & 18.18 & 41 & 15.53 \\
\hline $\begin{array}{l}\text { Agriculture is a tradition in my } \\
\text { family }\end{array}$ & 107 & 40.84 & 46 & 17.56 & 26 & 9.92 & 60 & 22.90 & 23 & 8.78 \\
\hline $\begin{array}{l}\text { Choosing an agriculturally related } \\
\text { career is expected of me }\end{array}$ & 175 & 66.54 & 43 & 16.35 & 12 & 4.56 & 9 & 3.42 & 24 & 9.13 \\
\hline
\end{tabular}


Table 12 (continued)

Family Influence

\begin{tabular}{|c|c|c|c|c|c|c|c|c|c|c|}
\hline & \multicolumn{2}{|c|}{$\begin{array}{l}\text { Not at All } \\
\text { Influential }\end{array}$} & \multicolumn{2}{|c|}{$\begin{array}{l}\text { Mildly } \\
\text { Influential }\end{array}$} & \multicolumn{2}{|c|}{$\begin{array}{l}\text { Moderately } \\
\text { Influential }\end{array}$} & \multicolumn{2}{|c|}{$\begin{array}{l}\text { Highly } \\
\text { Influential }\end{array}$} & \multicolumn{2}{|c|}{ Not Applicable } \\
\hline & $\mathrm{N}$ & $\%$ & $\mathrm{~N}$ & $\%$ & $\mathrm{~N}$ & $\%$ & $\mathrm{~N}$ & $\%$ & $\mathrm{~N}$ & $\%$ \\
\hline $\begin{array}{l}\text { My family encouraged me to } \\
\text { make the choice }\end{array}$ & 103 & 39.16 & 53 & 20.15 & 47 & 17.87 & 49 & 18.63 & 11 & 4.18 \\
\hline $\begin{array}{l}\text { I wanted to do something my } \\
\text { family has never done }\end{array}$ & 104 & 39.69 & 47 & 17.94 & 32 & 12.21 & 58 & 22.14 & 21 & 8.02 \\
\hline $\begin{array}{l}\text { An Agricultural Professional } \\
\text { influenced my choice }\end{array}$ & 110 & 42.31 & 31 & 11.92 & 45 & 17.31 & 52 & 20.00 & 22 & 8.46 \\
\hline $\begin{array}{l}\text { Extension Professional } \\
\text { influenced my choice }\end{array}$ & 147 & 56.32 & 43 & 16.48 & 22 & 8.43 & 22 & 8.43 & 27 & 10.34 \\
\hline $\begin{array}{l}\text { This decision was made to make } \\
\text { my parents proud }\end{array}$ & 122 & 46.39 & 59 & 22.43 & 43 & 16.35 & 26 & 9.89 & 13 & 4.94 \\
\hline $\begin{array}{l}\text { My parents insisted on an } \\
\text { agriculturally related career }\end{array}$ & 203 & 77.19 & 26 & 9.89 & 7 & 2.66 & 5 & 1.90 & 22 & 8.37 \\
\hline $\begin{array}{l}\text { My significant others } \\
\text { encouragement influenced my } \\
\text { decision }\end{array}$ & 182 & 68.94 & 20 & 7.58 & 13 & 4.92 & 9 & 3.41 & 40 & 15.15 \\
\hline
\end{tabular}




\section{Self-Motivations}

The second construct was self-motivations and included the following statements: "an agriculturally related career will satisfy my interests," "I am able to work in an agriculturally related career," "I want to work with my hands," "I enjoy physical labor," "I like creating innovative ways to perform tasks related to my career," "I like creating innovative ways to improve quality of life," "I enjoy growing food crops," "I like producing a product for public utilization," "I want to feed people," "I want to design new crops," "I want to manage pests in agriculture and forestry settings," "I like science," "I have a drive to succeed," and "I wanted to try something different." Also included in the construct self-motivations were: "I want to improve animal breeds," "I have a passion to help others better understand agriculture," "I have a passion to work with animals," "my dreams are agriculturally centered," "I want to work outdoors," "I like field work," "I enjoy working on new design projects," "I am self-motivated," "I want to help others help themselves" and "I want to become a leading innovator."

In response to the statement "an agriculturally relate career will satisfy my interests"; 20 students (8.55\%) reported this had no influence on their decision, while 20 students $(6.6 \%)$ were mildly influenced, 39 participants $(12.9 \%)$ were moderately influenced, and 145 students (47.9\%) were highly influenced because they felt an agriculturally related career will satisfy their interests (see Table 14). While 10 students (3.3\%) reported this to be inapplicable to their situation.

In regard to the statement, "I am able to work in an agriculturally related career"; $20(6.6 \%)$, respondents reported this to have no influence while 28 students $(9.2 \%)$ were 
mildly influenced, 49 individuals (16.2\%) were moderately influenced, and 123 participants $(40.6 \%)$ were highly influenced by their ability to work in an agriculturally related career (see Table 14 ). While 14 students (4.6\%) reported this did not apply to their situation.

In response to the statement, "I want to work with my hands" as an influential factor; 20 individuals $(6.6 \%)$ reported this had no influence on them, while 19 students $(6.3 \%)$ were mildly influenced, 47 individuals (15.5\%) were moderately influenced, and 142 students $(46.9 \%)$ were highly influenced because they want to work with their hands (see Table 14). While four individuals (1.3\%) reported this did not apply to their situation.

When asked whether, "I enjoy physical labor" was influential in their career choice; 24 participants (7.9\%) reported this had no influence on them, while 29 students (9.6\%) were mildly influenced, 78 students (25.7\%) were moderately influenced, and 95 individuals (31.4\%) were highly influenced by the fact they enjoy physical labor (see Table 14). While seven students $(2.3 \%)$ indicated this was inapplicable to their situation. In response to the statement "I like creating innovative ways to perform tasks" related to my career; nine students $(3.0 \%)$ reported this was not influential in their career decision, while 27 students (8.9\%) were mildly influenced, 83 students $(27.4 \%)$ were moderately influenced, and 110 students (36.3\%) were highly influenced by liking to create innovative ways to perform tasks (see Table 14). While five individuals (1.7\%) reported this does not pertain to their situation.

In regard to the statement "I like creating innovative ways to improve quality of life" as an influencing factor; 12 individuals (4.0\%) reported this was not influential, 
while 16 students (5.3\%) were mildly influenced, 73 individuals (24.1\%) were moderately influenced, and 128 students (42.2\%) were highly influenced by liking to create innovative ways to perform tasks (see Table 14). While six students $(2.0 \%)$ reported this to be inapplicable to their career choice.

In response to the statement "I enjoy growing food crops" as an influence; 54 participants (17.8\%) reported this was not influential, while 45 students (14.9\%) were mildly influenced, 51 students (16.8\%) were moderately influenced, and 69 participants (22.8\%) were highly influenced by the fact they growing their own food crops (see Table 14). While 15 students (5.0\%) reported this was inapplicable.

When asked if the statement "I like producing a product for public utilization" was a factor in their career choice; 42 individuals (13.9\%) reported this was not influential, while 44 students (14.5\%) were mildly influenced, 57 students (18.8\%) were moderately influenced, and 80 individuals (26.4\%) were highly influenced by producing a product for public utilization (see Table 14 ). While 12 students $(4.0 \%)$ reported this to be inapplicable to their situation.

In response to how influential the statement, "I want to feed people" was to their career choice; 61 participants (20.1\%) reported this was not influential, while 44 students (14.5\%) were mildly influenced, 47 individuals (15.5\%) were moderately influenced, and 65 students (21.5\%) were highly influenced by wanting to feed people (see Table 14). While 18 individuals (5.9\%) reported it to be inapplicable to their situation.

In response to the statement "I want to design new crops" being influential on career choice; 99 individuals (32.7\%) reported this was not influential, while 62 students (20.5\%) were mildly influenced, 26 students (8.6\%) were moderately influenced, and 22 
individuals (7.3\%) were highly influenced by wanting to design new crops (see Table 14). While 25 individuals ( $8.3 \%$ ) reported this did not pertain to their situation.

In regard to the statement "I want to manage pests in agriculture and forestry settings" as being a factor in career choice; 92 participants (30.4\%) reported this was not influential, while 45 individuals (14.9\%) were mildly influenced, 46 students (15.2\%) were moderately influenced, and 33 students (10.9\%) were highly influenced by wanting to manage pests in agriculture and forestry settings (see Table 14). While 20 individuals $(6.6 \%)$ reported this to be inapplicable to their situation.

When asked to rate the influence of the statement "I like science" on career choice; 19 participants $(6.3 \%)$ reported this was not influential, while 30 students $(9.9 \%)$ were mildly influenced, 60 individuals (19.8\%) were moderately influenced, and 117 individuals (38.6\%) were highly influenced by liking science (see Table 14). While ten participants (3.3\%) reported this to not pertain to their situation.

The degree "I have a drive to succeed" influenced career choice; seven individuals $(2.3 \%)$ reported this was not influential, 11 participants $(3.6 \%)$ were mildly influenced, 40 individuals (13.2\%) were moderately influenced, and 167 participants (55.1\%) were highly influenced by a drive to succeed (see Table 14). While $11(3.6 \%)$ reported this to be inapplicable to their situation.

"I want to try something different" was reported by 28 participants $(9.2 \%)$ as not influential, 37 individuals (12.2\%) reported it to be mildly influential, 61 students (20.1\%) were moderately influenced, and 93 individuals (30.7\%) were highly influenced by wanting to try something different (see Table 14). While 16 participants $(5.3 \%)$ reported this to not pertain to their circumstances. 
The degree to which "I want to improve animal breeds" influenced career choice; 86 participants (28.4\%) were not influenced by this, 41 individuals (13.5\%) were mildly influenced, 29 individuals (9.6\%) were moderately influenced, and 57 students (18.8\%) were highly influenced by wanting to improve animal breeds (see Table 14). While 22 participants $(7.3 \%)$ reported this to not be applicable to their circumstances.

In response to how influential the statement "I have a passion to help others better understand agriculture" was on career choice; 46 individuals (15.2\%) were not influenced by this, 39 participants (12.9\%) were mildly influenced, 40 individuals (13.2) were moderately influenced, and 96 participants (31.7\%) were highly influenced by having a passion to help others better understand agriculture (see Table 14). While 14 individuals (4.6\%) reported this did not apply to their situation.

When asked to what degree "I have a passion to work with animals" influenced career choice; 52 participants (17.2\%) were not influenced by this statement. However, the item had mild influence on 25 individuals (8.3\%), moderate influence on 39 students (12.9\%), and high influence on 90 individuals (29.7\%) who have a passion to work with animals (see Table 14). Twenty-eight individuals (11.97\%) reported this to be inapplicable to their situation.

With regard to the statement "My dreams are agriculturally centered"; 58 participants (19.1\%) were not influenced in their career choice by this statement, while having dreams that are agriculturally centered was mildly influential for 32 individuals (10.6\%), moderately influential for 41 participants (13.5\%), and highly influential for 89 individuals (29.4\%) (see Table 14). While 16 students (5.3\%) reported this as inapplicable to their situation. 
In response to how influential "I want to work outdoors" was to their career decision, 21 participants (6.9\%) reported this was not influential, while 24 individuals (7.9\%) were mildly influenced, 37 participants $(12.2 \%)$ were moderately influenced, and 140 individuals (46.2\%) were highly influenced by wanting to work outdoors (see Table 14). While 13 students (4.3\%) reported this as not applicable.

When responding to the influence "I like field work" was on career choice, 29 participants (9.6\%) reported it as not influential, while 23 participants (7.6\%) were mildly influenced, 44 participants (14.5\%) were moderately influenced, and 122 individuals (40.3\%) were highly influenced by liking field work (see Table 14). While sixteen students (5.3\%) reported this to not apply to their situation.

With regard to the level of influence the statement "I enjoy working on new design projects" had on career choice, 34 participants (11.2\%) reported they were not influenced, while 44 individuals (14.5\%) were mildly influenced, 70 participants (23.1\%) were moderately influenced, and 76 individuals (25.1\%) were highly influenced by enjoying work on new design projects (see Table 14). While this was inapplicable for 11 participants (3.6\%).

The statement "I am self-motivated" was reported by seven (2.3\%) individuals as not influential, while ten participants (3.3\%) were mildly influenced, 67 participants (22.1\%) were moderately influenced, and 138 participants (45.5\%) were highly influenced by self-motivation (see Table 14). This was not applicable for 12 individuals $(4.0 \%)$.

The amount that "I want to help others help themselves" influenced career choice was reported by 20 participants (6.6\%) as not influential, while 28 individuals (9.2\%) 
were mildly influenced, 54 participants $(17.8 \%)$ were moderately influenced, and 123 individuals (40.6\%) were highly influenced by wanting to help others help themselves (see Table 14). This was not applicable for ten individuals (3.3\%).

When responding to the influence "I want to become a leading innovator" had on career choice, 28 individuals $(9.2 \%)$ reported they were not influenced, while 43 participants (14.2\%) were mildly influenced, 55 individuals (18.2\%) were moderately influenced, and 96 students (31.7\%) were highly influenced by wanting to become a leading innovator (see Table 14). While 13 students (4.3\%) reported this to not apply to their situation.

\section{Constructs - Self-Motivation}

The Likert items were combined into composite constructs. The scale for the composite constructs is as follows: Not Influential $=1-1.5$, Mildly Influential $=1.6-$ 2.5, Moderately Influential $=2.6-3.5$, and Highly Influential $=3.6$ and above. SelfMotivations was rated overall as moderately influential $(\mathrm{M}=2.98, \mathrm{SD}=.62)$ (see Table 13 or Table 21).

Table 13

Construct - Self Motivation

\begin{tabular}{lcccc}
\hline & M & SD & Min & Max \\
\hline Self-Motivations & 2.98 & .62 & 1.00 & 4.00 \\
\hline
\end{tabular}


Table 14

Self-Motivations

\begin{tabular}{|c|c|c|c|c|c|c|c|c|c|c|}
\hline & \multicolumn{2}{|c|}{$\begin{array}{l}\text { Not at All } \\
\text { Influential }\end{array}$} & \multicolumn{2}{|c|}{ Mildly Influential } & \multicolumn{2}{|c|}{$\begin{array}{l}\text { Moderately } \\
\text { Influential }\end{array}$} & \multicolumn{2}{|c|}{ Highly Influential } & \multicolumn{2}{|c|}{ NA } \\
\hline & $\mathrm{N}$ & $\%$ & $\mathrm{~N}$ & $\%$ & $\mathrm{~N}$ & $\%$ & $\mathrm{~N}$ & $\%$ & $\mathrm{~N}$ & $\%$ \\
\hline $\begin{array}{l}\text { An agriculturally related career will } \\
\text { satisfy my interests }\end{array}$ & 20 & 8.55 & 20 & 8.55 & 39 & 16.67 & 145 & 61.97 & 10 & 4.27 \\
\hline $\begin{array}{l}\text { I am able to work in an agriculturally } \\
\text { related career }\end{array}$ & 20 & 8.55 & 28 & 11.97 & 49 & 20.94 & 123 & 52.56 & 14 & 5.98 \\
\hline I want to work with my hands & 20 & 8.62 & 19 & 8.19 & 47 & 20.26 & 142 & 61.21 & 4 & 1.72 \\
\hline I enjoy physical labor & 24 & 10.30 & 29 & 12.45 & 78 & 33.48 & 95 & 40.77 & 7 & 3.00 \\
\hline $\begin{array}{l}\text { I like creating innovative ways to } \\
\text { perform tasks related to my career }\end{array}$ & 9 & 3.85 & 27 & 11.54 & 83 & 35.47 & 110 & 47.01 & 5 & 2.14 \\
\hline $\begin{array}{l}\text { I like creating innovative ways to } \\
\text { improve quality of life }\end{array}$ & 12 & 5.11 & 16 & 6.81 & 73 & 31.06 & 128 & 54.47 & 6 & 2.55 \\
\hline I enjoy growing food crops & 54 & 23.08 & 45 & 19.23 & 51 & 21.79 & 69 & 29.49 & 15 & 6.41 \\
\hline $\begin{array}{l}\text { I like producing a product for public } \\
\text { utilization }\end{array}$ & 42 & 17.87 & 44 & 18.72 & 57 & 24.26 & 80 & 34.04 & 12 & 5.11 \\
\hline I want to feed people & 61 & 25.96 & 44 & 18.72 & 47 & 20.00 & 65 & 27.66 & 18 & 7.66 \\
\hline
\end{tabular}


Table 14 (continued)

Self-Motivations

\begin{tabular}{|c|c|c|c|c|c|c|c|c|c|c|}
\hline & \multicolumn{2}{|c|}{$\begin{array}{l}\text { Not at All } \\
\text { Influential }\end{array}$} & \multicolumn{2}{|c|}{ Mildly Influential } & \multicolumn{2}{|c|}{$\begin{array}{l}\text { Moderately } \\
\text { Influential }\end{array}$} & \multicolumn{2}{|c|}{ Highly Influential } & \multicolumn{2}{|c|}{ NA } \\
\hline & $\mathrm{N}$ & $\%$ & $\mathrm{~N}$ & $\%$ & $\mathrm{~N}$ & $\%$ & $\mathrm{~N}$ & $\%$ & $\mathrm{~N}$ & $\%$ \\
\hline I want to design new crops & 99 & 42.31 & 62 & 26.50 & 26 & 11.11 & 22 & 9.40 & 25 & 10.68 \\
\hline $\begin{array}{l}\text { I want to manage pests in agriculture } \\
\text { and forestry settings }\end{array}$ & 92 & 38.98 & 45 & 19.07 & 46 & 19.49 & 33 & 13.98 & 20 & 8.47 \\
\hline I like science & 19 & 8.05 & 30 & 12.71 & 60 & 25.42 & 117 & 49.58 & 10 & 4.24 \\
\hline I have a drive to succeed & 7 & 2.97 & 11 & 4.66 & 40 & 16.95 & 167 & 70.76 & 11 & 4.66 \\
\hline I wanted to try something different & 28 & 11.91 & 37 & 15.74 & 61 & 25.96 & 93 & 39.57 & 16 & 6.81 \\
\hline I want to improve animal breeds & 86 & 36.60 & 41 & 17.45 & 29 & 12.34 & 57 & 24.26 & 22 & 9.36 \\
\hline $\begin{array}{l}\text { I have a passion to help others better } \\
\text { understand agriculture }\end{array}$ & 46 & 19.57 & 39 & 16.60 & 40 & 17.02 & 96 & 40.85 & 14 & 5.96 \\
\hline I have a passion to work with animals & 52 & 22.22 & 25 & 10.68 & 39 & 16.67 & 90 & 38.46 & 28 & 11.97 \\
\hline My dreams are agriculturally centered & 58 & 24.58 & 32 & 13.56 & 41 & 17.37 & 89 & 37.71 & 16 & 6.78 \\
\hline I want to work outdoors & 21 & 8.94 & 24 & 10.21 & 37 & 15.74 & 140 & 59.57 & 13 & 5.53 \\
\hline
\end{tabular}


Table 14 (continued)

Self-Motivations

\begin{tabular}{|c|c|c|c|c|c|c|c|c|c|c|}
\hline & \multicolumn{2}{|c|}{$\begin{array}{l}\text { Not at All } \\
\text { Influential }\end{array}$} & \multicolumn{2}{|c|}{ Mildly Influential } & \multicolumn{2}{|c|}{$\begin{array}{l}\text { Moderately } \\
\text { Influential }\end{array}$} & \multicolumn{2}{|c|}{ Highly Influential } & \multicolumn{2}{|c|}{ NA } \\
\hline & $\mathrm{N}$ & $\%$ & $\mathrm{~N}$ & $\%$ & $\mathrm{~N}$ & $\%$ & $\mathrm{~N}$ & $\%$ & $\mathrm{~N}$ & $\%$ \\
\hline I like field work & 29 & 12.39 & 23 & 9.83 & 44 & 18.80 & 122 & 52.14 & 16 & 6.84 \\
\hline $\begin{array}{l}\text { I enjoy working on new design } \\
\text { projects }\end{array}$ & 34 & 14.47 & 44 & 18.72 & 70 & 29.79 & 76 & 32.34 & 11 & 4.68 \\
\hline I am self-motivated & 7 & 2.99 & 10 & 4.27 & 67 & 28.63 & 138 & 58.97 & 12 & 5.13 \\
\hline I want to help others help themselves & 20 & 8.51 & 28 & 11.91 & 54 & 22.98 & 123 & 52.34 & 10 & 4.26 \\
\hline I want to become a leading innovator & 28 & 11.91 & 43 & 18.30 & 55 & 23.40 & 96 & 40.85 & 13 & 5.53 \\
\hline
\end{tabular}




\section{Experience}

The third construct was experiences and included the following statements: "I have worked on a farm," "I have worked in an agricultural business," "I have family pursuing careers in agriculturally related fields," "I learned about potential agricultural careers through media outlets," "I participated in agricultural course(s) in high school," "I have prior experience in agriculture," "My 4-H project work influenced my career choice," and "My participation in National FFA convention influenced my career choice."

With regard to how influential the statement "I have worked on a farm" was to career choice; 57 individuals $(18.8 \%)$ reported this was not influential, while it was mildly influential for 32 participants (10.6\%), moderately influential for 26 individual (8.6\%), and highly influential for 81 participants $(26.7 \%)$ due to having worked on a farm (see Table 16). This was not applicable for 30 individuals (9.9\%).

"I have worked in an agricultural business" was reported to have been not influential for 73 participants $(24.1 \%)$, while 24 participants $(7.9 \%)$ were mildly influenced, 28 individuals (9.2\%) were moderately influenced, and 60 individuals $(19.8 \%)$ were highly influenced by having worked in an agricultural business (see Table 16). While 39 individuals (12.9\%) reported this to inapplicable.

"I have family pursuing careers in agriculturally related fields" was reported by 104 individuals $(34.3 \%)$ to have not been influential, while 24 participants $(7.9 \%)$ reported it as mildly influential, 19 students (6.3\%) were moderately influenced, and 29 
students $(9.6 \%)$ were highly influenced by family pursuing careers in agriculturally related fields (see Table 16). This was reported as not applicable for 46 students (15.2\%).

"I learned about potential agricultural careers through media outlets" was reported by 94 students (31.0\%) as not influential in their career choice, while 53 participants (17.5\%) were mildly influenced, 28 participants $(9.2 \%)$ were moderately influenced, and 21 students $(6.9 \%)$ were highly influenced by learning of potential agricultural careers through media outlets (see Table 16). Thirty participants (13.27\%) reported this does not apply to their situation.

"I participated in agricultural course(s) in high school" was reported by 83 students $(27.4 \%)$ to have not been influential in their career choice, while 19 participants (6.3\%) were mildly influenced, 11 individuals (3.6\%) were moderately influenced, and 65 individuals (21.5\%) were highly influenced by participating in agricultural courses in high school (see Table 16). This was not applicable for 47 participants (15.5\%).

In response to the statement "I have prior experience in agriculture" and its influence on career choice; 61 participants (20.1\%) were not influenced by this, while 33 individuals (10.9\%) were mildly influenced, 32 students (10.6\%) were moderately influenced, and 71 students (23.4\%) were highly influenced by having prior experience in agriculture (see Table 16). This was not applicable for 29 students $(9.6 \%)$.

"My 4-H project work influenced my career choice" was reported by 96 individuals (31.7\%) as not influential, while 13 individuals (4.3\%) reported it to be mildly influential, and $13(4.3 \%)$ reported it to be moderately influential, and 30 participants $(9.9 \%)$ were highly influenced in their career choice by 4-H project work (see Table 16). While 73 individuals (32.44\%) reported this to be inapplicable. 
"My participation in National FFA convention" as influential in their career choice was reported by 88 students $(29.0 \%)$ to have not been influential, while 10 students (3.3\%) were mildly influenced, 13 individuals $(4.3 \%)$ were moderately influenced, and 40 students (13.2\%) were highly influenced by their participation in National FFA convention (see Table 16). This was reported as not applicable by 76 individuals (25.1\%).

\section{Constructs - Experiences}

The Likert items were combined into composite constructs. The scale for the composite constructs is as follows: Not Influential $=1-1.5$, Mildly Influential $=1.6-$ 2.5, Moderately Influential $=2.6-3.5$, and Highly Influential $=3.6$ and above.

Experiences was rated overall with a score of moderately influential $(\mathrm{M}=2.24, \mathrm{SD}=.98)$ (see Table 15 or Table 21).

Table 15

Construct - Experience

\begin{tabular}{lcccc}
\hline & M & SD & Min & Max \\
\hline Experiences & 2.24 & .98 & 1.00 & 4.00 \\
\hline
\end{tabular}


Table 16

Experiences

\begin{tabular}{|c|c|c|c|c|c|c|c|c|c|c|}
\hline & \multicolumn{2}{|c|}{$\begin{array}{l}\text { Not at All } \\
\text { Influential }\end{array}$} & \multicolumn{2}{|c|}{ Mildly Influential } & \multicolumn{2}{|c|}{$\begin{array}{l}\text { Moderately } \\
\text { Influential }\end{array}$} & \multicolumn{2}{|c|}{ Highly Influential } & \multicolumn{2}{|c|}{ NA } \\
\hline & $\mathrm{N}$ & $\%$ & $\mathrm{~N}$ & $\%$ & $\mathrm{~N}$ & $\%$ & $\mathrm{~N}$ & $\%$ & $\mathrm{~N}$ & $\%$ \\
\hline I have worked on a farm & 57 & 25.22 & 32 & 14.16 & 26 & 11.50 & 81 & 35.84 & 30 & 13.27 \\
\hline $\begin{array}{l}\text { I have worked in an agricultural } \\
\text { business }\end{array}$ & 73 & 2.59 & 24 & 10.71 & 28 & 12.50 & 60 & 26.79 & 39 & 17.41 \\
\hline $\begin{array}{l}\text { I have family pursuing careers in } \\
\text { agriculturally related fields }\end{array}$ & 104 & 46.85 & 24 & 10.81 & 19 & 8.56 & 29 & 13.06 & 46 & 20.72 \\
\hline $\begin{array}{l}\text { I learned about potential agricultural } \\
\text { careers through media outlets }\end{array}$ & 94 & 41.59 & 53 & 23.45 & 28 & 12.39 & 21 & 9.29 & 30 & 13.27 \\
\hline $\begin{array}{l}\text { I participated in agricultural } \\
\text { course(s) in high school }\end{array}$ & 83 & 36.89 & 19 & 8.44 & 11 & 4.89 & 65 & 28.89 & 47 & 20.89 \\
\hline $\begin{array}{l}\text { I have prior experience in } \\
\text { agriculture }\end{array}$ & 61 & 26.99 & 33 & 14.60 & 32 & 14.16 & 71 & 31.42 & 29 & 12.83 \\
\hline $\begin{array}{l}\text { My 4-H project work influenced my } \\
\text { career choice }\end{array}$ & 96 & 42.67 & 13 & 5.78 & 13 & 5.78 & 30 & 13.33 & 73 & 32.44 \\
\hline $\begin{array}{l}\text { My participation in National FFA } \\
\text { convention influenced career choice }\end{array}$ & 88 & 38.77 & 10 & 4.41 & 13 & 5.73 & 40 & 17.62 & 76 & 33.48 \\
\hline
\end{tabular}




\section{Use of Skills}

The fourth construct Use of Skills included the following statements: "Career options in my chosen field are enjoyable," "Careers in agriculture allow for me to make an impact," "My career choice allows me to use problem solving strategies," "My career choice allows me to use verbal communication skills," "My career allows me to use written communication skills," "My career allows me to demonstrate managerial skills," "My career allows me to demonstrate leadership skills," "My career allows me to work with people," "My career allows the opportunity to work with people of different cultures," "My career allows me to work with environmental issues," "My career allows me to use computer skills," "My career allows me to use technical skills," "I want a career with room to move-up the ladder," "My career provides the opportunity to work with highly knowledgeable professionals," and "My career choice offers a variety of challenging activities."

In response to the degree of influence the statement "Career options in my chosen field are enjoyable" had on career choice; seven individuals $(2.3 \%)$ responded this was not influential, while 11 participants (3.6\%) were mildly influenced, 45 participants (14.9\%) were moderately influenced, and 143 students (47.2\%) were highly influenced by career options in their chosen field being enjoyable (see Table 18). This was not applicable for five participants $(1.7 \%)$.

"Careers in agriculture allow for me to make an impact" was reported by 23 individuals (7.6\%) as not influencing their career choice, while 26 individuals $(8.6 \%)$ were mildly influenced, 39 participants $(12.9 \%)$ were moderately influenced, and 106 
participants (35.0\%) were highly influenced by being allowed to make an impact with their career choice (see Table 18). This was inapplicable for 15 individuals (5.0\%).

In response to the influence "My career choice allows me to use problem solving strategies" had on career choice was reported by nine individuals (3.0\%) as not being influential, while 28 individuals (9.2\%) were mildly influenced, 62 participants (20.5\%) were moderately influenced, 104 participants (34.3\%) were highly influenced by their career choice allowing them to use problem solving strategies (see Table 18). This was reported as inapplicable for five individuals (1.7\%).

"My career choice allows me to use verbal communication skills" was reported by 18 individuals (5.9\%) as not influential in their career choice, while 18 participants (5.9\%) were mildly influenced, 62 participants $(20.5 \%)$ were moderately influenced, and 108 participants (35.6\%) were highly influenced by the use of verbal communication skills in their chosen field (see Table 18). This was not applicable for four individuals $(1.3 \%)$.

In response to how influential "My career allows me to use written communication skills" was on career choice 27 individuals (8.9\%) reported it was not influential, while 37 students (12.2\%) were mildly influenced, 58 students (19.1\%) were moderately influenced, and 83 participants (27.4\%) were highly influenced by the use of written communication skills in their chosen career (see Table 18). This was not applicable for four individuals (1.3\%).

In regard to how influential the statement "My career allows me to demonstrate managerial skills" was in career choice 25 participants (8.3\%) reported it was not influential, while 31 participants (10.2\%) were mildly influenced, 59 students (19.5\%) 
were moderately influenced, and 90 students (29.7\%) were highly influenced by the opportunity to demonstrate managerial skills in their chosen career (see Table 18). This was reported as not applicable by five individuals (1.7\%).

"My career allows me to demonstrate leadership skills" was reported by 18 participants (5.9\%) as not influential in determining career choice, while 21 students (6.9\%) were mildly influenced, 42 participants (13.9\%) were moderately influenced, and 124 individuals (40.9\%) were highly influenced by the opportunity to demonstrate leadership skills in their chosen career (see Table 18). This was not applicable for four individuals $(1.3 \%)$.

"My career allows me to work with people" was not influential for 17 students $(5.6 \%)$ in making career choice, while 23 individuals (7.6\%) were mildly influenced, 43 participants (14.2\%) were moderately influenced, and 122 participants $(40.3 \%)$ were highly influenced by a career with the opportunity to work with people (see Table 18). Four participants $(1.3 \%)$ reported this was inapplicable to their situation.

"My career allows the opportunity to work with people of different cultures" was reported by 32 individuals $(10.6 \%)$ as having no influence on their career choice, while 33 respondents $(10.9 \%)$ were mildly influenced, 60 individuals $(19.8 \%)$ were moderately influenced, and 78 students $(25.7 \%)$ were highly influenced by the opportunity to work with people of different cultures in their chosen career (see Table 18). Seven students $(2.3 \%)$ reported it was inapplicable to their situation.

In response to the statement "My career allows me to work with environmental issues" 18 respondents (5.9\%) reported this did not an influence their career choice, while 36 participants (11.9\%) were mildly influenced, 53 participants $(17.5 \%)$ were moderately 
influenced, and 93 students (30.7\%) were highly influenced by the opportunity to work with environmental issues (see Table 18). This was reported as inapplicable by eight students $(2.6 \%)$.

"My career allows me to use computer skills" was reported by 47 students (15.5\%) as not being influential in their career decision, while 48 students (15.8\%) reported it as mildly influential, 50 participants (16.5\%) reported moderate influence, and 57 students (18.8 \%) were highly influenced by the use of computer skills in their chosen career (see Table 18). Seven individuals (2.3\%) reported this was not applicable to their situation.

"My career allows me to use technical skills" was reported by 32 individuals $(10.6 \%)$ as not influential in their career choice, while 42 students (13.9\%) were mildly influenced, 60 individuals (19.8\%) were moderately influenced, and 68 students (22.4\%) were highly influenced by the use of technical skills in their chosen career (see Table 18). Six students (2.0\%) reported this was not applicable to their situation.

"I want a career with room to move-up the ladder" was reported by 28 respondents $(9.2 \%)$ as having no influence on career choice, while 30 individuals $(9.9 \%)$ were mildly influenced, 52 participants (17.2\%) were moderately influenced, and 88 individuals $(29.0 \%)$ were highly influenced in their career choice by the desire to have a career with room to move-up the ladder (see Table 18). This was reported as not applicable by eight students (2.6\%).

"My career provides the opportunity to work with highly knowledgeable professionals" was reported as not influential in their career decision by 12 respondents (4.0\%), while 20 participants (6.6\%) were mildly influenced, 45 students $(14.9 \%)$ were 
moderately influenced, and 127 students (41.9\%) were highly influenced by the opportunity to work with highly knowledgeable professionals in their career (see Table 18). Six students (2.0\%) reported this was not applicable to their situation.

"My career choice offers a variety of challenging activities" was reported by 10 respondents (3.3\%) to not be influential in their career decision, while nine students (3.0\%) were mildly influenced, 53 respondents (17.5\%) were moderately influenced, and 130 individuals (42.9\%) were highly influenced by a variety of challenging activities within their career (see Table 18). Seven students (2.3\%) reported this was not applicable to their situation.

\section{Constructs - Use of Skills}

The Likert items were combined into composite constructs. The scale for the composite constructs is as follows: Not Influential $=1-1.5$, Mildly Influential $=1.6-$ 2.5, Moderately Influential $=2.6-3.5$, and Highly Influential $=3.6$ and above. Use of Skills was rated moderately influential ((M=3.17, $\mathrm{SD}=.69)$ (see Table 17 or Table 21). Table 17

Construct - Use of Skills

\begin{tabular}{lcccc}
\hline & M & SD & Min & Max \\
\hline Use of Skills & 3.17 & .69 & 1.00 & 4.00 \\
\hline
\end{tabular}


Table 18

Use of Skills

\begin{tabular}{|c|c|c|c|c|c|c|c|c|c|c|}
\hline & \multicolumn{2}{|c|}{$\begin{array}{l}\text { Not at All } \\
\text { Influential }\end{array}$} & \multicolumn{2}{|c|}{ Mildly Influential } & \multicolumn{2}{|c|}{$\begin{array}{l}\text { Moderately } \\
\text { Influential }\end{array}$} & \multicolumn{2}{|c|}{ Highly Influential } & \multicolumn{2}{|c|}{ NA } \\
\hline & $\mathrm{N}$ & $\%$ & $\mathrm{~N}$ & $\%$ & $\mathrm{~N}$ & $\%$ & $\mathrm{~N}$ & $\%$ & $\mathrm{~N}$ & $\%$ \\
\hline $\begin{array}{l}\text { Career options in my chosen field } \\
\text { are enjoyable }\end{array}$ & 7 & 3.32 & 11 & 5.21 & 45 & 21.33 & 143 & 67.77 & 5 & 2.37 \\
\hline $\begin{array}{l}\text { Careers in agriculture allow for me } \\
\text { to make an impact }\end{array}$ & 23 & 11.00 & 26 & 12.44 & 39 & 18.66 & 106 & 50.72 & 15 & 7.18 \\
\hline $\begin{array}{l}\text { My career choice allows me to use } \\
\text { problem solving strategies }\end{array}$ & 9 & 4.33 & 28 & 13.46 & 62 & 29.81 & 104 & 50.00 & 5 & 2.40 \\
\hline $\begin{array}{l}\text { My career choice allows me to use } \\
\text { verbal communication skills }\end{array}$ & 18 & 8.57 & 18 & 8.57 & 62 & 29.52 & 108 & 51.43 & 4 & 1.90 \\
\hline $\begin{array}{l}\text { My career allows me to use written } \\
\text { communication skills }\end{array}$ & 27 & 12.92 & 37 & 17.70 & 58 & 27.75 & 83 & 39.71 & 4 & 1.91 \\
\hline $\begin{array}{l}\text { My career allows me to demonstrate } \\
\text { managerial skills }\end{array}$ & 25 & 11.90 & 31 & 14.76 & 59 & 28.10 & 90 & 42.86 & 5 & 2.38 \\
\hline $\begin{array}{l}\text { My career allows me to demonstrate } \\
\text { leadership skills }\end{array}$ & 18 & 8.61 & 21 & 10.05 & 42 & 20.10 & 124 & 59.33 & 4 & 1.91 \\
\hline $\begin{array}{l}\text { My career allows me to work with } \\
\text { people }\end{array}$ & 17 & 8.13 & 23 & 11.00 & 43 & 20.57 & 122 & 58.37 & 4 & 1.91 \\
\hline
\end{tabular}


Table 18 (continued)

Use of Skills

\begin{tabular}{|c|c|c|c|c|c|c|c|c|c|c|}
\hline & \multicolumn{2}{|c|}{$\begin{array}{l}\text { Not at All } \\
\text { Influential }\end{array}$} & \multicolumn{2}{|c|}{ Mildly Influential } & \multicolumn{2}{|c|}{$\begin{array}{l}\text { Moderately } \\
\text { Influential }\end{array}$} & \multicolumn{2}{|c|}{ Highly Influential } & \multicolumn{2}{|c|}{ NA } \\
\hline & $\mathrm{N}$ & $\%$ & $\mathrm{~N}$ & $\%$ & $\mathrm{~N}$ & $\%$ & $\mathrm{~N}$ & $\%$ & $\mathrm{~N}$ & $\%$ \\
\hline $\begin{array}{l}\text { My career allows me to work with } \\
\text { people of different cultures }\end{array}$ & 32 & 15.24 & 33 & 15.71 & 60 & 28.57 & 78 & 37.14 & 7 & 3.33 \\
\hline $\begin{array}{l}\text { My career allows me to work with } \\
\text { environmental issues }\end{array}$ & 18 & 8.65 & 36 & 17.31 & 53 & 25.48 & 93 & 44.71 & 8 & 3.85 \\
\hline $\begin{array}{l}\text { My career allows me to use } \\
\text { computer skills }\end{array}$ & 47 & 22.49 & 48 & 22.97 & 50 & 23.92 & 57 & 27.27 & 7 & 3.35 \\
\hline $\begin{array}{l}\text { My career allows me to use } \\
\text { technical skills }\end{array}$ & 32 & 15.38 & 42 & 20.19 & 60 & 28.85 & 68 & 32.69 & 6 & 2.88 \\
\hline $\begin{array}{l}\text { I want a career with room to move- } \\
\text { up the ladder }\end{array}$ & 28 & 13.59 & 30 & 14.56 & 52 & 25.24 & 88 & 42.72 & 8 & 3.88 \\
\hline $\begin{array}{l}\text { My career provides the opportunity } \\
\text { to work with highly knowledgeable } \\
\text { professionals }\end{array}$ & 12 & 5.71 & 20 & 9.52 & 45 & 21.43 & 127 & 60.48 & 6 & 2.86 \\
\hline $\begin{array}{l}\text { My career choice offers a variety of } \\
\text { challenging activities }\end{array}$ & 10 & 4.78 & 9 & 4.31 & 53 & 25.36 & 130 & 62.20 & 7 & 3.35 \\
\hline
\end{tabular}




\section{Career Opportunities}

Construct five was developed using items that promoted career opportunities and included the following statements: "Agricultural careers have multiple opportunities," "An agriculturally related career would give me financial security," "Agriculturally related careers would allow me to support my family," "The current economic situation factored in my decision to select an agriculturally related field," "Income motivated me to choose this field of work," "An agriculturally related career would improve my feeling of self-worth," "There is potential for employment directly after college," "Grant money available to support career endeavors after graduation," "There are a number of job opportunities in desirable locations," "There are a number of job opportunities in my home state," "The size of the industry allows for many career opportunities," "There are many career opportunities associated with my degree," and "My career choice has great job benefits."

“Agricultural careers have multiple opportunities" was reported by 12 individuals $(4.0 \%)$ as not influential on their career choice, while 15 students $(5.0 \%)$ were mildly influenced, 44 participants (14.5\%) were moderately influenced, and 110 respondents (36.3\%) were highly influenced by agricultural careers having multiple opportunities (see Table 20$)$. Twelve individuals (4.0\%) reported this was not applicable for their situation.

"An agriculturally related career would give me financial security" was reported by 32 individuals $(10.6 \%)$ as not influential in their career decision, while 47 students (15.5\%) were mildly influenced, 57 individuals (18.8\%) were moderately influenced, and 43 students (14.2\%) were highly influenced by financial security (see Table 20). Thirteen individuals $(4.3 \%)$ reported this was not applicable to their situation. 
In response to the statement that "Agriculturally related careers would allow me to support me family" 22 students $(7.3 \%)$ reported this was not influential in their career choice, while 40 individuals (13.2\%) were mildly influenced, 55 students (18.2\%) were moderately influenced, and 57 respondents (18.8\%) were highly influenced by being able to support their families through an agriculturally related career (see Table 20). Seventeen participants (5.6\%) reported this was not applicable to their situation.

"The current economic situation factored in my decision to select an agriculturally related field" was reported by 74 students $(24.4 \%)$ as not being an influential factor, while 39 participants (12.9\%) were mildly influenced, 30 students $(9.9 \%)$ were moderately influenced, and 30 students $(9.9 \%)$ were highly influenced by the current economic situation (see Table 20). Nineteen individuals (6.3\%) reported this was not applicable to their circumstances.

"Income motivated me to choose this field of work" 71 participants (23.4\%) reported this was not influential, while 49 students (16.2\%) were mildly influenced, 45 participants (14.9\%) were moderately influenced, and 20 students $(6.6 \%)$ were highly influenced by the income in this field of work (see Table 20). Eight students (2.6\%) reported this was not applicable to their situation.

"An agriculturally related career would improve my feeling of self-worth" was reported by 27 respondents $(8.9 \%)$ as not influential in their career choice, while 35 students (11.6\%) were mildly influenced, 40 students (13.2\%) were moderately influenced, and 73 participants (24.1\%) were highly influenced by an agricultural career improving their feelings of self-worth (see Table 20). Fifteen respondents (5.0\%) reported this was not applicable to their circumstances. 
The amount of influence "There is potential for employment directly after college" had on career decision was reported by 18 students (5.9\%) as not influential, while 36 participants (11.9\%) were mildly influenced, 55 respondents (18.2\%) were moderately influenced, and 78 individuals (25.7\%) were highly influenced by potential employment directly after college (see Table 20$)$. Six students (2.0\%) reported this was not applicable to their situation.

"Grant money available to support career endeavors after graduation" was reported by 49 respondents $(16.2 \%)$ as having no influence on their career choice, while 50 students (16.5\%) were mildly influenced, 41 participants (13.5\%) were moderately influenced, and 32 students (10.6\%) were highly influenced by available grant money to support career endeavors after graduation (see Table 20). Twenty-one students (6.9\%) reported this was not applicable to their situation.

"There are a number of job opportunities in desirable locations" was considered not influential by 25 individuals (8.3\%), while 31 students (10.2\%) were mildly influenced, 49 students (16.2\%) were moderately influenced, and 82 participants (27.1\%) were highly influenced by the number of job opportunities in desirable locations (see Table 20). Six students (2.0\%) reported this was not applicable to their circumstances.

"There are a number of job opportunities in my home state" was reported by 35 respondents $(11.6 \%)$ as not influential in their career choice, while 36 participants (11.9\%) were mildly influenced, 49 respondents $(16.2 \%)$ were moderately influenced, and 65 individuals $(21.5 \%)$ were highly influenced by the number of job opportunities in their home state (see Table 20). Eight students (2.6\%) reported this was not applicable to their situation. 
"The size of the industry allows for many career opportunities" was considered by 22 students (7.3\%) as not influential in career decision, while 36 students (11.9\%) were mildly influenced, 58 students (19.1\%) were moderately influenced, and 72 students (23.8\%) were highly influenced by the size of the industry allowing for many career opportunities (see Table 20). Five individuals (1.7\%) reported this was not applicable to their situation.

"There are many career opportunities associated with my degree" was considered not to be influential in their career choice by 15 students $(5.0 \%)$, while 22 respondents (7.3\%) were mildly influenced, 52 students (17.2\%) were moderately influenced, and 98 students (32.3\%) were highly influenced by the career opportunities associated with their degree (see Table 20). Six respondents (2.0\%) reported this was not applicable to their situation.

The statement "My career choice has great job benefits" was reported by 25 students $(8.3 \%)$ as not influential in career decision, while 38 students $(12.5 \%)$ were mildly influenced, 45 students (14.9\%) were moderately influenced, and 77 respondents (25.4\%) were highly influenced by great job benefits (see Table 20). Eight students (2.6\%) reported this was not applicable to their situations.

\section{Constructs - Career Opportunities}

The Likert items were combined into composite constructs. The scale for the composite constructs is as follows: Not Influential $=1-1.5$, Mildly Influential $=1.6-$ 2.5, Moderately Influential $=2.6-3.5$, and Highly Influential $=3.6$ and above. Career Opportunities was also rated overall as moderately influential $(\mathrm{M}=2.80, \mathrm{SD}=.74)$ (see Table 19 or Table 21). 
Table 19

Construct - Career Opportunities

\begin{tabular}{lcccc}
\hline & M & SD & Min & Max \\
\hline Career Opportunities & 2.80 & .74 & 1.00 & 4.00 \\
\hline
\end{tabular}


Table 20

\section{Career Opportunities}

\begin{tabular}{|c|c|c|c|c|c|c|c|c|c|c|}
\hline & \multicolumn{2}{|c|}{$\begin{array}{l}\text { Not at All } \\
\text { Influential }\end{array}$} & \multicolumn{2}{|c|}{ Mildly Influential } & \multicolumn{2}{|c|}{$\begin{array}{l}\text { Moderately } \\
\text { Influential }\end{array}$} & \multicolumn{2}{|c|}{ Highly Influential } & \multicolumn{2}{|c|}{ NA } \\
\hline & $\mathrm{N}$ & $\%$ & $\mathrm{~N}$ & $\%$ & $\mathrm{~N}$ & $\%$ & $\mathrm{~N}$ & $\%$ & $\mathrm{~N}$ & $\%$ \\
\hline $\begin{array}{l}\text { Agricultural careers have multiple } \\
\text { opportunities }\end{array}$ & 12 & 6.22 & 15 & 7.77 & 44 & 22.80 & 110 & 56.99 & 12 & 6.22 \\
\hline $\begin{array}{l}\text { An agriculturally related career } \\
\text { would give me financial security }\end{array}$ & 32 & 16.67 & 47 & 24.48 & 57 & 29.69 & 43 & 22.40 & 13 & 6.77 \\
\hline $\begin{array}{l}\text { Agriculturally related careers would } \\
\text { allow me to support my family }\end{array}$ & 22 & 11.52 & 40 & 20.94 & 55 & 28.80 & 57 & 29.84 & 17 & 8.90 \\
\hline $\begin{array}{l}\text { The current economic situation } \\
\text { factored in my decision to select an } \\
\text { agriculturally related field }\end{array}$ & 74 & 38.54 & 39 & 20.31 & 30 & 15.63 & 30 & 15.63 & 19 & 9.90 \\
\hline $\begin{array}{l}\text { Income motivated me to choose this } \\
\text { field of work }\end{array}$ & 71 & 36.79 & 49 & 25.39 & 45 & 23.32 & 20 & 10.36 & 8 & 4.15 \\
\hline $\begin{array}{l}\text { An agriculturally related career } \\
\text { would improve my feeling of self- } \\
\text { worth }\end{array}$ & 27 & 14.21 & 35 & 18.42 & 40 & 21.05 & 73 & 38.42 & 15 & 7.89 \\
\hline $\begin{array}{l}\text { There is potential for employment } \\
\text { directly after college }\end{array}$ & 18 & 9.33 & 36 & 18.65 & 55 & 28.50 & 78 & 40.41 & 6 & 3.11 \\
\hline
\end{tabular}


Table 20 (continued)

Career Opportunities

\begin{tabular}{|c|c|c|c|c|c|c|c|c|c|c|}
\hline & \multicolumn{2}{|c|}{$\begin{array}{l}\text { Not at All } \\
\text { Influential }\end{array}$} & \multicolumn{2}{|c|}{ Mildly Influential } & \multicolumn{2}{|c|}{$\begin{array}{l}\text { Moderately } \\
\text { Influential }\end{array}$} & \multicolumn{2}{|c|}{ Highly Influential } & \multicolumn{2}{|c|}{ NA } \\
\hline & $\mathrm{N}$ & $\%$ & $\mathrm{~N}$ & $\%$ & $\mathrm{~N}$ & $\%$ & $\mathrm{~N}$ & $\%$ & $\mathrm{~N}$ & $\%$ \\
\hline $\begin{array}{l}\text { Grant money available to support } \\
\text { career endeavors after graduation }\end{array}$ & 49 & 25.39 & 50 & 25.91 & 41 & 21.24 & 32 & 16.58 & 21 & 10.88 \\
\hline $\begin{array}{l}\text { There are a number of job } \\
\text { opportunities in desirable locations }\end{array}$ & 25 & 12.95 & 31 & 16.06 & 49 & 25.39 & 82 & 42.49 & 6 & 3.11 \\
\hline $\begin{array}{l}\text { There are a number of job } \\
\text { opportunities in my home state }\end{array}$ & 35 & 18.13 & 36 & 18.65 & 49 & 25.39 & 65 & 33.68 & 8 & 4.15 \\
\hline $\begin{array}{l}\text { The size of the industry allows for } \\
\text { many career opportunities }\end{array}$ & 22 & 11.40 & 36 & 18.65 & 58 & 30.05 & 72 & 37.31 & 5 & 2.59 \\
\hline $\begin{array}{l}\text { There are many career opportunities } \\
\text { associated with my degree }\end{array}$ & 15 & 7.77 & 22 & 11.40 & 52 & 26.94 & 98 & 50.78 & 6 & 3.11 \\
\hline $\begin{array}{l}\text { My career choice has great job } \\
\text { benefits }\end{array}$ & 25 & 12.95 & 38 & 19.69 & 45 & 23.32 & 77 & 39.90 & 8 & 4.15 \\
\hline
\end{tabular}




\section{Constructs - Overall}

The Likert items were combined into composite constructs. The scale for the composite constructs is as follows: Not Influential $=1-1.5$, Mildly Influential $=1.6-$ 2.5, Moderately Influential $=2.6-3.5$, and Highly Influential $=3.6$ and above (see Table 21).

Table 21

Construct - Overall Summary

\begin{tabular}{lcccc}
\hline & $\mathrm{M}$ & SD & Min & Max \\
\hline Family Influence & 1.87 & .58 & 1.00 & 3.86 \\
Self-Motivations & 2.98 & .62 & 1.00 & 4.00 \\
Experiences & 2.24 & .98 & 1.00 & 4.00 \\
Use of Skills & 3.17 & .69 & 1.00 & 4.00 \\
Career Opportunities & 2.80 & .74 & 1.00 & 4.00 \\
\hline
\end{tabular}

\section{Top Five Family Influences}

The five constructs were analyzed to determine the top five motivational items of each construct. Moderately influential and highly influential participants were summed together to find the top five most influential items of each construct. Family influence construct consisted of fifteen items. "Teachers influenced my decision" was the top rated factor in the construct by 102 individuals (38.63\%). "An Agricultural Professional influenced my choice" ranked seconded highest in the construct by 97 individuals (37.31\%). "My family encouraged me to make the choice" ranked third in the construct by 96 students (36.5\%). "My parents influenced my decision" ranked fourth by 94 
respondents (35.61\%). "I wanted to do something my family has never done" ranked fifth in the construct by 90 individuals (34.35\%) (see Table 22 ).

Table 22

Top Five Family Influences

\begin{tabular}{lcc}
\hline & $\mathrm{N}$ & $\%$ \\
\hline Teachers influenced my decision & 102 & 38.63 \\
An Agricultural Professional influenced my choice & 97 & 37.31 \\
My family encouraged me to make the choice & 96 & 36.5 \\
My parents influenced my decision & 94 & 35.61 \\
I wanted to do something my family has never done & 90 & 34.35 \\
\hline
\end{tabular}

\section{Top Five Self-Motivators}

The second construct, self-motivators consisted of 24 items. "I have a drive to succeed" ranked first in the construct with 207 individuals (87.71\%). The second highest ranking was "I am self-motivated" with 205 respondents (87.6\%). "I like creating innovative ways to improve quality of life" was third highest in the construct by 201 individuals $(85.53 \%)$. "I like creating innovative ways to perform tasks related to my career" ranked fourth in the construct by 193 participants $(82.48 \%)$. "I want to work with my hands" ranked fifth highest within the construct by 189 individuals (81.47\%) (see Table 23). 
Table 23

Top Five Self-Motivators

\begin{tabular}{|c|c|c|}
\hline & $\mathrm{N}$ & $\%$ \\
\hline I have a drive to succeed & 207 & 87.71 \\
\hline I am self-motivated & 205 & 87.6 \\
\hline $\begin{array}{l}\text { I like creating innovative ways to improve } \\
\text { quality of life }\end{array}$ & 201 & 85.53 \\
\hline $\begin{array}{l}\text { I like creating innovative ways to perform tasks } \\
\text { related to my career }\end{array}$ & 193 & 82.48 \\
\hline I want to work with my hands & 189 & 81.47 \\
\hline
\end{tabular}

\section{Top Five Experiences}

The third construct 'Experiences' was comprised of eight items. "I have worked on a farm" was rated number one in the construct by 107 individuals (47.34\%). "I have prior experiences in agriculture" was second with 103 participants (45.58\%), while "I have worked in an agricultural business" ranked third by 88 respondents (39.29\%). "I participated in agricultural course(s) in high school" ranked fourth in the construct by 76 individuals (33.78\%), followed in fifth place by "My participation in National FFA convention influenced my career choice" by 53 individuals (23.35\%) (see Table 24). 
Table 24

Top Five Experiences

\begin{tabular}{lcc}
\hline & $\mathrm{N}$ & $\%$ \\
\hline I have worked on a farm & 107 & 47.34 \\
I have prior experience in agriculture & 103 & 45.58 \\
I have worked in an agricultural business & 88 & 39.29 \\
$\begin{array}{l}\text { I participated in agricultural course(s) in high } \\
\text { school }\end{array}$ & 76 & 33.78 \\
$\begin{array}{l}\text { My participation in National FFA convention } \\
\text { influenced my career choice }\end{array}$ & 53 & 23.35 \\
\hline
\end{tabular}

\section{Top Five Use of Skills}

'Use of Skills' construct was comprised of 15 items. "Career options in my chosen field are enjoyable" was rated number one by 188 individuals (89.1\%). "My career choice offers a variety of challenging activities" was second with 183 students (87.56\%), while "My career provides the opportunity to work with highly knowledgeable professionals" ranked third in the construct by 172 students $(81.91 \%)$. "My career choice allows me to use verbal communication skills ranked fourth by 170 students $(80.95 \%)$ while "My career choice allows me to use problem solving strategies" ranked fifth in the construct by 166 individuals (79.81\%) (see Table 25$)$. 
Table 25

Top Five Use of Skills

\begin{tabular}{lcc}
\hline & $\mathrm{N}$ & $\%$ \\
\hline $\begin{array}{l}\text { Career options in my chosen field are } \\
\text { enjoyable }\end{array}$ & 188 & 89.1 \\
$\begin{array}{l}\text { My career choice offers a variety of } \\
\text { challenging activities }\end{array}$ & 183 & 87.56 \\
$\begin{array}{l}\text { My career provides the opportunity to work } \\
\text { with highly knowledgeable professionals }\end{array}$ & 172 & 81.91 \\
$\begin{array}{l}\text { My career choice allows me to use verbal } \\
\text { communication skills }\end{array}$ & 170 & 80.95 \\
$\begin{array}{l}\text { My career choice allows me to use problem } \\
\text { solving strategies }\end{array}$ & 166 & 79.81 \\
\hline
\end{tabular}

\section{Top Five Career Opportunities}

The fifth construct 'career opportunities' consisted of 13 items with “Agricultural careers have multiple opportunities" rated number one by 154 students $(79.79 \%)$. "There are many career opportunities associated with my degree" ranked second by 150 students (77.72\%) while "There is potential for employment directly after college" ranked third in the construct by 133 participants $(68.91 \%)$. “There are a number of job opportunities in desirable locations" was rated fourth by 131 students $(67.8 \%)$ and the fifth ranked item was "The size of the industry allows for many career opportunities" with 130 individuals (67.36\%) reporting it as highly or moderately influential (see Table 26). 
Table 26

Top Five Career Opportunities

\begin{tabular}{lcc}
\hline & $\mathrm{N}$ & $\%$ \\
\hline Agricultural careers have multiple opportunities & 154 & 79.79 \\
$\begin{array}{l}\text { There are many career opportunities associated with } \\
\text { my degree }\end{array}$ & 150 & 77.72 \\
$\begin{array}{l}\text { There is potential for employment directly after college } \\
\text { There are a number of job opportunities in desirable } \\
\text { locations }\end{array}$ & 133 & 68.91 \\
$\begin{array}{l}\text { The size of the industry allows for many career } \\
\text { opportunities }\end{array}$ & 131 & 67.88 \\
\hline
\end{tabular}

\section{Relationship between having worked on a Farm or Ranch and Pursuing an}

\section{Agriculturally Related Career}

A chi-square test of independence was used to determine if there was a significant relationship between having worked on a farm or ranch and pursuing an agriculturally related career. The hypotheses included:

$\mathrm{H}_{0}$ : Having worked on a farm or ranch and pursuing an agriculturally related career are independent of one another.

$\mathrm{H}_{1}$ : There is an association between having worked on a farm or ranch and pursuing an agriculturally related career.

The chi-square value was $25.81(\mathrm{df}=1)$ which determined there was a significant difference. The null hypothesis was rejected and the alternative hypothesis accepted that there was an association between having worked on a farm or ranch and pursuing an agriculturally related career (see Table 27). 
The participants who had prior work experience on a farm pursuing an agriculturally related career predicted count was 88.3 with a higher actual count of 103 . Those with farm or ranch experience who were not pursuing an agricultural career predicted count was higher at 26.7 with a 12 actual count (see Table 27).

Likewise, those pursuing an agriculturally related career with no farm or ranch experience had a predicted count of 60.7 and an actual count of 43 . Students not pursuing an agriculturally related career with no experience on a farm or ranch had a predicted count of 18.3 and an actual count of 33 (see Table 27). 
Table 27

Chi Square Analysis- Worked on a Farm or Ranch and Pursuing an Agriculturally Related Career

\begin{tabular}{|c|c|c|c|c|c|}
\hline & & & \multicolumn{3}{|c|}{ Pursuing an Agriculturally Related Career } \\
\hline & & & Yes & No & Total \\
\hline \multirow{6}{*}{$\begin{array}{l}\text { Worked on a } \\
\text { Farm or Ranch }\end{array}$} & Yes & Count & 103 & 12 & 115 \\
\hline & & Expected Count & 88.3 & 26.7 & 115.0 \\
\hline & & $\%$ within Worked on a Farm or Ranch & $89.6 \%$ & $10.4 \%$ & $100.0 \%$ \\
\hline & No & Count & 46 & 33 & 79 \\
\hline & & Expected Count & 60.7 & 18.3 & 79.0 \\
\hline & & $\%$ within Worked on a Farm or Ranch & $58.2 \%$ & $41.8 \%$ & $100.0 \%$ \\
\hline \multirow[t]{3}{*}{ Total } & & Count & 149 & 45 & 194 \\
\hline & & Expected Count & 149.0 & 45.0 & 194.0 \\
\hline & & $\%$ within Worked on a Farm or Ranch & $76.8 \%$ & $23.2 \%$ & $100.0 \%$ \\
\hline
\end{tabular}




\section{Relationship between having worked in an Agricultural Business and Pursuing an}

\section{Agriculturally Related Career}

A chi-square test of independence was used to determine if a significant

relationship existed between having worked in an agricultural business and pursuing an agriculturally related career. The hypotheses are as stated:

$\mathrm{H}_{0}$ : Having worked in an agricultural business and pursing an agriculturally related career are independent of one another.

$\mathrm{H}_{1}$ : There is a relationship between having worked in an agricultural business and pursuing an agriculturally related career.

The chi-square value was $10.61(\mathrm{df}=1)$ determined there was a significant difference. The null hypothesis was rejected and the alternative hypothesis was accepted. There was an association between having worked in an agricultural business and pursuing an agriculturally related career (see Table 28).

Students pursuing an agriculturally related career who have worked in an agricultural business predicted count of 52.8 with an actual count of 62. Students who have not worked in an agricultural business pursuing an agriculturally related career predicted count was 94.2 with an actual count of 85 (see Table 28).

Respondents not pursing an agriculturally related career who have worked in an agricultural business predicted count of 16.2 with an actual count of 7 . Students neither pursuing an agricultural career nor having worked in an agricultural business had a predicted count of 28.8 with a higher actual count of 38 (see Table 28). 
Table 28

Chi-square Analysis: Worked in Agricultural Business and Pursuing an Agriculturally Related Career

\begin{tabular}{|c|c|c|c|c|c|}
\hline & & & \multicolumn{3}{|c|}{ Pursuing an Agriculturally Related Career } \\
\hline & & & Yes & No & Total \\
\hline \multirow{8}{*}{$\begin{array}{l}\text { Worked in an } \\
\text { Agricultural } \\
\text { Business }\end{array}$} & Yes & Count & 62 & 7 & 69 \\
\hline & & Expected Count & 52.8 & 16.2 & 69.0 \\
\hline & & $\%$ within Worked in an Agricultural & & & \\
\hline & & Business & $89.9 \%$ & $10.1 \%$ & $100.0 \%$ \\
\hline & No & Count & 85 & 38 & 123 \\
\hline & & Expected Count & 94.2 & 28.8 & 123.0 \\
\hline & & $\%$ within Worked in an Agricultural & & & \\
\hline & & Business & $69.1 \%$ & $30.9 \%$ & $100.0 \%$ \\
\hline \multirow[t]{3}{*}{ Total } & & Count & 147 & 45 & 192 \\
\hline & & Expected Count & 147.0 & 45.0 & 192.0 \\
\hline & & $\begin{array}{l}\text { \% within Worked in an Agricultural } \\
\text { Business }\end{array}$ & $76.6 \%$ & $23.4 \%$ & $100.0 \%$ \\
\hline
\end{tabular}




\section{Relationship between having Relatives Working on a Farm or Ranch and Pursuing an Agriculturally Related Career}

A chi-square test of independence was used to determine if a significant relationship existed between relatives working on a farm or ranch and pursuing an agriculturally related career. The hypotheses are as stated:

$\mathrm{H}_{0}$ : Relatives working on a farm or ranch and pursuing an agriculturally related career are independent of one another.

$\mathrm{H}_{1}$ : There is an association between relatives working on a farm or ranch and pursuing an agriculturally related career.

The chi-square value was $11.57(\mathrm{df}=1)$ determined there was a significance difference. The null hypothesis was rejected and the alternative hypothesis was accepted. There was an association between relatives working on a farm or ranch and pursuing an agriculturally related career (see Table 29).

Students pursuing an agricultural career along with relatives with experience on a farm or ranch had a predicted count of 93.4 and an actual count of 103 . Students without relatives having work experience on a farm or ranch who were pursuing an agriculturally related career predicted count of 55.6 with an actual count of 46 (see Table 29).

Students who were not pursuing an agriculturally related career, but did have relatives working on a farm or ranch had a predicted count of 27.6 and an actual count of 18. Students who were not pursuing an agriculturally related career along with not having relatives with experience on a farm or ranch had a predicted count of 16.4 and an actual count of 26 (see Table 29). 
Table 29

Chi-square Analysis: Relatives Working on a Farm or Ranch and Pursing an Agriculturally Related Career

\begin{tabular}{|c|c|c|c|c|c|}
\hline & & & \multicolumn{3}{|c|}{ Pursuing an Agriculturally Related Career } \\
\hline & & & Yes & No & Total \\
\hline \multirow{6}{*}{$\begin{array}{l}\text { Relatives Working } \\
\text { on a Farm-Ranch }\end{array}$} & Yes & Count & 103 & 18 & 121 \\
\hline & & Expected Count & 93.4 & 27.6 & 121.0 \\
\hline & & $\begin{array}{l}\% \text { within Relatives Working on a } \\
\text { Farm-Ranch }\end{array}$ & $85.1 \%$ & $14.9 \%$ & $100.0 \%$ \\
\hline & No & Count & 46 & 26 & 72 \\
\hline & & Expected Count & 55.6 & 16.4 & 72.0 \\
\hline & & $\begin{array}{l}\text { \% within Relatives Working on a Farm- } \\
\text { Ranch }\end{array}$ & $63.9 \%$ & $36.1 \%$ & $100.0 \%$ \\
\hline \multirow[t]{3}{*}{ Total } & & Count & 149 & 44 & 13 \\
\hline & & Expected Count & 149.0 & 44.0 & 193.0 \\
\hline & & $\begin{array}{l}\% \text { within Relatives Working on a } \\
\text { Farm-Ranch }\end{array}$ & $77.2 \%$ & $22.8 \%$ & $100.0 \%$ \\
\hline
\end{tabular}




\section{Relationship between having Relatives Working in an Agricultural Business and Pursuing an Agriculturally Related Career}

A chi-square test was used to determine if a significant relationship existed between relatives working in an agricultural business and pursuing an agriculturally related career. The hypotheses are as stated:

$\mathrm{H}_{0}$ : Relatives working in an agricultural business and pursing an agriculturally related career are independent of one another.

$\mathrm{H}_{1}$ : The alternative hypothesis: There is an association between relatives working in an agricultural business and pursuing an agriculturally related career.

The chi-square value was $9.89(\mathrm{df}=1)$. This value was significantly different thus we rejected the null hypothesis and accepted the alternative hypothesis. There was an association between relatives working in an agricultural business and pursuing an agriculturally related career (see Table 30).

Students pursuing an agricultural career along with relatives with experience in an agricultural business had a predicted count of 59.0 and an actual count of 68 . Students without relatives having work experience on a farm or ranch who were pursuing an agriculturally related career predicted count of 88.0 with an actual count of 78 (see Table $30)$.

Students who were not pursuing an agriculturally related career, but did have relatives working on a farm or ranch had a predicted count of 18.0 and an actual count of 9. Students who were not pursuing an agriculturally related career along with not having relatives with experience on a farm or ranch had a predicted count of 27.0 and an actual count of 36 (see Table 30). 
Table 30

Chi-Square Analysis: Relatives Working in an Agricultural Business and Pursing an Agriculturally Related Career

\begin{tabular}{|c|c|c|c|c|c|}
\hline & & & \multicolumn{3}{|c|}{ Pursuing an Agriculturally Related Career } \\
\hline & & & Yes & No & Total \\
\hline \multirow{6}{*}{$\begin{array}{l}\text { Relatives Working } \\
\text { in an Agricultural } \\
\text { Business }\end{array}$} & \multirow[t]{3}{*}{ Yes } & Count & 68 & 9 & 77 \\
\hline & & Expected Count & 59.0 & 18.0 & 77.0 \\
\hline & & $\begin{array}{l}\% \text { within Relatives Working in an } \\
\text { Agricultural Business }\end{array}$ & $88.3 \%$ & $11.7 \%$ & $100.0 \%$ \\
\hline & \multirow[t]{3}{*}{ No } & Count & 79 & 36 & 115 \\
\hline & & Expected Count & 88.0 & 27.0 & 115.0 \\
\hline & & $\begin{array}{l}\text { \% within Relatives Working in an } \\
\text { Agricultural Business }\end{array}$ & $68.7 \%$ & $31.3 \%$ & $100.0 \%$ \\
\hline \multirow[t]{3}{*}{ Total } & & Count & 147 & 45 & 192 \\
\hline & & Expected Count & 147.0 & 45.0 & 192.0 \\
\hline & & $\begin{array}{l}\text { \% within Relatives Working in an } \\
\text { Agricultural Business }\end{array}$ & $76.6 \%$ & $23.4 \%$ & $100.0 \%$ \\
\hline
\end{tabular}




\section{Relationship between Taking High School Agricultural Course and Pursuing an Agriculturally Related Career}

A chi-square test of independence was used to determine if a significant relationship existed between taking high school agricultural courses and pursuing an agriculturally related career. The hypotheses are as stated:

$\mathrm{H}_{0}$ : Taking high school agricultural courses and pursuing an agriculturally related career are independent of one another.

$\mathrm{H}_{1}$ : There is an association between taking high school agricultural courses and pursuing an agriculturally related career.

The chi-square value of $25.99(\mathrm{df}=1)$ indicates a significant difference. The null hypothesis was rejected and the alternative hypothesis was accepted that there was an association between taking high school agricultural courses and pursuing an agriculturally related career (see Table 31 ).

Students who have taken high school agricultural courses and are pursuing agriculturally related careers had a predicted count of 58.4 and an actual count of 73 . Participants who have taken high school agricultural courses and are not pursuing agriculturally related careers had a predicted count of 17.6 and an actual count of 3 (see Table 31).

Students who did not have high school agricultural courses and are pursuing agriculturally related careers had a predicted count of 90.6 and an actual count of 76. Participants who did not have high school agricultural courses and are not pursuing agriculturally related careers had a predicted count of 27.4 and an actual count of 42 (see Table 31). 
Table 31

Chi-Square Analysis: High School Agricultural Courses and Pursuing an Agriculturally Related Career

\begin{tabular}{|c|c|c|c|c|c|}
\hline & & & \multicolumn{3}{|c|}{ Pursuing an Agriculturally Related Career } \\
\hline & & & Yes & No & Total \\
\hline \multirow{6}{*}{$\begin{array}{l}\text { High School } \\
\text { Agricultural } \\
\text { Courses }\end{array}$} & Yes & Count & 73 & 3 & 76 \\
\hline & & Expected Count & 58.4 & 17.6 & 76.0 \\
\hline & & $\begin{array}{l}\% \text { within High School Agricultural } \\
\text { Courses }\end{array}$ & $96.1 \%$ & $3.9 \%$ & $100.0 \%$ \\
\hline & No & Count & 76 & 42 & 118 \\
\hline & & Expected Count & 90.6 & 27.4 & 118.0 \\
\hline & & $\begin{array}{l}\% \text { within High School Agricultural } \\
\text { Courses }\end{array}$ & $64.4 \%$ & $35.6 \%$ & $100.0 \%$ \\
\hline \multirow[t]{3}{*}{ TOTAL } & & Count & 149 & 45 & 194 \\
\hline & & Expected Count & 149.0 & 45.0 & 194.0 \\
\hline & & $\begin{array}{l}\% \text { within High School Agricultural } \\
\text { Courses }\end{array}$ & $76.8 \%$ & $23.2 \%$ & $100.0 \%$ \\
\hline
\end{tabular}




\section{Relationship between College Agriculturally Related Organization Involvement and Pursuing an Agriculturally Related Career}

A chi-square test of independence was used to determine if a significant relationship existed between college agriculturally related organization involvement and pursing an agriculturally related career. The hypotheses are as stated:

$\mathrm{H}_{0}$ : College agriculturally related organization involvement and pursing an agriculturally related career are independent of one another.

$\mathrm{H}_{1}$ : There is an association between college agriculturally related organization involvement and pursuing an agriculturally related career.

The chi-square value of $21.00(\mathrm{df}=1)$ determined there was a significant

difference. The null hypothesis was rejected and the alternative hypothesis was accepted. There was an association between college agricultural organization involvement and pursuing an agriculturally related career (see Table 32).

Students with involvement in college agricultural organizations pursuing an agriculturally related career predicted count of 66.6 with an actual count of 80 .

Participants not involved with college agricultural organizations pursuing an agriculturally related career predicted count of 80.4 and an actual count of 67 (see Table 32).

Students involved with college agricultural organizations not pursuing an agriculturally related career had a predicted count of 20.4 and an actual count of 7 . Participants not involved with college agricultural organizations and not pursuing an agriculturally related career had a predicted count of 24.6 and an actual count of 38 (see Table 32). 
Table 32

Chi-Square Analysis: College Agricultural Organization Involvement and Pursuing an Agriculturally Related Career

\begin{tabular}{|c|c|c|c|c|c|}
\hline & & & \multicolumn{3}{|c|}{ Pursuing an Agriculturally Related Career } \\
\hline & & & Yes & No & Total \\
\hline \multirow{7}{*}{$\begin{array}{l}\text { College } \\
\text { Agriculturally } \\
\text { Related } \\
\text { Organization } \\
\text { Involvement }\end{array}$} & \multirow[t]{4}{*}{ Yes } & Count & 80 & 7 & 87 \\
\hline & & Expected Count & 66.6 & 20.4 & 87.0 \\
\hline & & \% within College A oriculturally Related & & & \\
\hline & & Organization Involvement & $92.0 \%$ & $8.0 \%$ & $100.0 \%$ \\
\hline & \multirow[t]{3}{*}{ No } & Count & 67 & 38 & 105 \\
\hline & & Expected Count & 80.4 & 24.6 & 105.0 \\
\hline & & $\begin{array}{l}\% \text { within College Agriculturally Related } \\
\text { Organization Involvement }\end{array}$ & $63.8 \%$ & $36.2 \%$ & $100 \%$ \\
\hline \multirow[t]{3}{*}{ Total } & & Count & 147 & 45 & 192 \\
\hline & & Expected Count & 147.0 & 45.0 & 192.0 \\
\hline & & $\begin{array}{l}\% \text { within College Agriculturally Related } \\
\text { Organization Involvement }\end{array}$ & $76.6 \%$ & $23.4 \%$ & $100.0 \%$ \\
\hline
\end{tabular}




\section{Relationship between Gender and Pursuing an Agriculturally Related Career}

A chi-square was used to determine if a significant relationship existed between gender and pursing an agriculturally related career. The hypotheses are as stated:

$\mathrm{H}_{0}$ : Gender and pursing an agriculturally related career are independent of one another.

$\mathrm{H}_{1}$ : There is a relationship between gender and pursuing an agriculturally related career

The chi square value of $1.60(\mathrm{df}=1)$ was not significantly different. Therefore, the null hypothesis failed to be rejected. Gender and pursuing an agriculturally related career were independent of one another (see Table 33). 
Table 33

Chi-Square Analysis: Gender and Pursing an Agriculturally Related Career

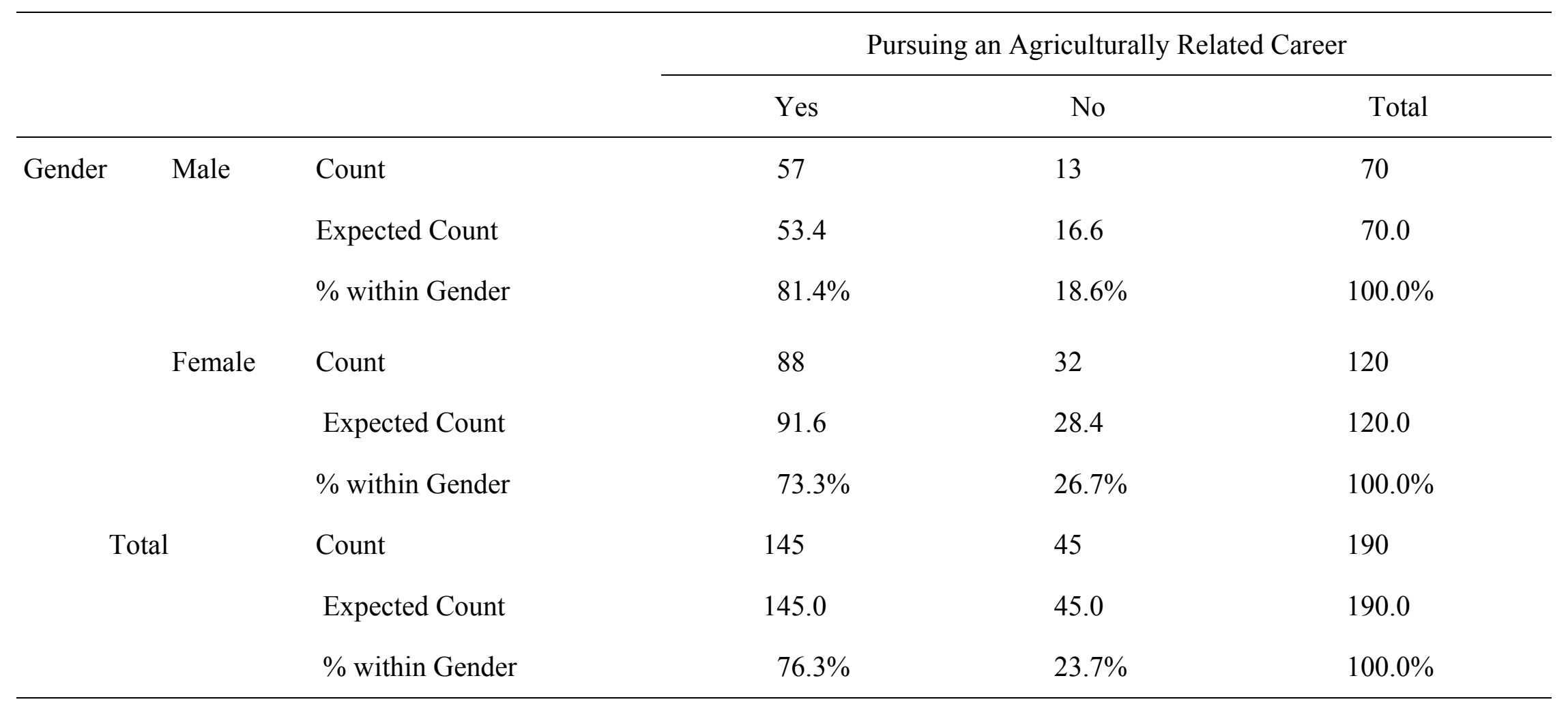




\section{CHAPTER V}

\section{Summary, Conclusions, and Recommendations}

\section{Purpose of the Study}

The purpose of this study is to determine the motivational factors contributing to an undergraduate student's choice to pursue an agriculturally related career. By determining factors which motivate students to choose agriculturally related careers, high school teachers, guidance counselors, extension agents, and colleges have a better understanding as to how to advise and recruit students to pursue agriculturally related careers.

\section{Objective of the Study}

The objective of the study was to determine the motivational factors associated with career choices of undergraduate students pursuing degrees in agriculturally related fields. The following research questions were used to guide this study:

1. What factors influenced students to pursue a career in an agriculturally related field?

2. Do students enrolled in an agricultural degree program anticipate pursuing a career in an agriculturally related field?

3. Which youth organizations have agricultural degree students participated in prior to college?

4. Is there an association between previous experience working on a farm or ranch and pursuing an agriculturally related career?

5. Is there an association between previous experience working in an agricultural business and pursuing an agriculturally related career? 
6. Is there an association between parents or relatives working in an agricultural business and pursuing an agriculturally related career?

7. Is there an association between parents or relatives working on a farm or ranch and pursuing an agriculturally related career?

8. Is there an association between gender and pursuing an agriculturally related career?

9. Is there an association between enrollment in high school agricultural courses and pursuing an agriculturally related career?

10. Is there an association between college agricultural related organization involvement courses and pursuing an agriculturally related career?

\section{Summary}

The study consisted of census of 1673 students enrolled in the Davis College of Agriculture, Natural Resources and Design at West Virginia University. The response rate was $18.11 \%$.

A majority of the respondents were female, and greater than $90 \%$ of the respondents were white. Slightly more than half of the respondents $52.1 \%$ were between the ages of 18 and 23. Two thirds of the respondents were from West Virginia. Over one-fourth of the population lives in small cities or suburban areas with a population of 5,000 to 50,000 people, while a third of the participants were from small farms or ranches. Nearly half of the respondents were senior status while more than a third of the participants carried a 3.0 to 3.49 grade point average. Slightly less than half of the respondents had been involved in 4-H or FFA as a youth. Each of the degree programs within the Davis College of Agriculture, Natural Resource and Design were represented 
through this study. The units with the highest levels of participation were Animal and Nutritional Science and Agricultural and Extension Education.

Two-thirds of the respondents reported they intend to pursue an agriculturally related career. Over half of the respondents reported they were very satisfied with their career choice. A majority of the students estimated their starting salary would be between $\$ 20,001$ and $\$ 60,000$ a year. One third of the respondents had an animal science background, while over half of the students had worked on a farm or ranch or had relatives working on a farm or ranch. Twenty percent of the respondents indicated their mothers/female guardians held careers in education, while the largest number of the fathers/male guardians was reported to hold positions as laborers, followed by positions in management.

The Likert items were divided into five constructs of Family Influences, SelfMotivators, Motivating Experiences, Motivations Involving Use of Skills, and Motivational Career Opportunities. The top five motivational factors of each construct were determined.

The top five family influences were: 'Teachers influenced my decision,' 'An Agricultural Professional influenced my choice,' 'My family encouraged me to make the choice,' 'My parents influenced my decision,' and 'I wanted to do something my family has never done,' respectively. These top five influences further support the findings of Wildman and Torres (2001), Sutphin and Newsom-Stewart (1995), Donnermeyer and Kreps (1994), Cole (1985), Findlay and Rawls (1984) Bowen and Lee (1984), Faulks (1969), Miller, Williams, \& Sprouse (1984), and Reis and Kahler (1997). Their studies 
reported that parents, family, teachers, counselors, and/or friends are highly motivating factors when deciding on career options.

The top five items for self-motivators were: 'I have a drive to succeed,' 'I am self-motivated,' 'I like creating innovative ways to improve quality of life,' 'I like creating innovative ways to perform tasks related to my career,' and 'I want to work with my hands,' respectively. These findings support the findings by Llopis (2012) identified motivating factors for the work place which included: being relevant, proving others wrong, career advancement, reaching for goals, making an impact, and happiness.

The top five motivating experiences were: 'I have worked on a farm,' 'I have prior experience in agriculture,' 'I have worked in an agricultural business,' 'I participated in agricultural course(s) in high school,' and 'My participation in National FFA convention influenced my career choice,' respectively. Several of the top five motivating experiences support findings by Pink (2008), Associated Press (2010), Ng (2008), and Reis and Kahler (1997). Their findings reported that interest in the work field was determined to be a contributing factor to choosing careers. Rawls, Martin, Negatu, \& Robertson (1994) found that career considerations can include the nature of the work (i.e., working outdoors, working with people and/or animals), availability and location of the job, and prestige of career area.

The top five motivations involving use of skills were: 'Career options in my chosen field are enjoyable,' 'My career choice offers a variety of challenging activities,' 'My career provides the opportunity to work with highly knowledgeable professionals,' 'My career choice allows me to use verbal communication skills,' and 'My career choice allows me to use problem solving strategies,' respectively. These statements are 
supported by previous study findings including interest in the work field (Pink, 2008; Associated Press, 2010; Ng, 2008; Reis and Kahler, 1997), good working conditions (Ng, 2008), reaching goals, making an impact, and happiness (Llopis, 2012), and fulfilling a purpose (Pink, 2009; Buckingham \& Clifton, 2001; Llopis, 2012) which have been identified as motivational factors contributing to choosing a career.

The top five motivational career opportunities in order were: 'agricultural careers have multiple opportunities,' 'there are many career opportunities associated with my degree,' 'there is potential for employment directly after college,' 'there are a number of job opportunities in desirable locations,' and 'the size of the industry allows for many career opportunities.' Previous studies by Martin, Milne-Home, Barrett, Spalding and Jones, (2000), National Research Council, (1996), Thomson, (1994), and Scanlon, Bruening, and Cordero, (1996), report that the field of Agriculture will need well versed individuals to take on contemporary changes. Goecker, Smith, Smith and Goetz (2010) found that agricultural, food, and renewable natural resources sectors of the U.S. economy will generate an estimated 54,400 annual openings for individuals with baccalaureate or higher degrees in food, renewable energy, and environmental specialties between 2010 and 2015. Llopis (2012) and $\mathrm{Ng}$ (2008) both reported that job stability was a contributing factor to choosing a career.

A significant relationship was found between 'pursuing an agriculturally related career': and the following factors including: having worked on a farm or ranch, worked in an agricultural business, relatives working on a farm or ranch, relatives working in an agricultural business, high school agricultural courses, and college agriculturally related 
organization involvement. Gender, was found to have no relationship with 'pursuing an agriculturally related career.'

\section{Conclusions}

Based on the results of this study, the following conclusions were made:

1. Teachers influenced career decisions of respondents.

2. Respondents have a drive to succeed.

3. Respondents are motivated by their experience on the farm.

4. Pursuit of an agriculturally related career is significantly impacted by having worked on a farm or ranch, having previous experience working in an agricultural business, relatives having worked on a farm or ranch, relatives having worked in an agricultural business, high school agricultural courses, and college agriculturally related organization involvement.

5. There was no relationship between gender and pursuing an agriculturally related career.

\section{Recommendations}

Recruiters looking for potential students should keep in mind the previous experiences those students have had in their lifetime. Those who have high potential, best suited for a college of agriculture have had experience on a farm or ranch or participated in agricultural courses and/or agricultural conventions. Recruiters may also stress that the agricultural industry is growing with 54,000 possible job opportunities over the next few years. These positions will need well educated employees to perform skilled tasks. 
Teachers and agricultural professionals are one of the top motivational factors related to students choosing careers in agriculturally related fields. Students must be enabled to learn about the different aspects of agriculture. Teachers and agricultural professionals should allow for hands on experience in agriculturally related fields of study. Through discussions, experience, positive influence, and the opportunity to become involved in agriculturally related organizations, teachers and agricultural professionals will allow students to see agriculture in a positive light therefore influencing, over time, their career choices towards agriculturally related fields.

This study should be replicated in other colleges of agriculture to see if the findings would be similar. 


\section{REFERENCES}

Adams, S. (2012, May 18). New survey: majority of employees dissatisfied. Retrieved from http://www.forbes.com/sites/susanadams/2012/05/18/new-

Associated Press. (2010, January 05). Survey: More Americans unhappy at work. Retrieved from http://www.cbsnews.com/news/survey-more-americans-unhappyat-work/

Ary, D., Jacobs, L. C., Sorensen, C. K., \& Walker, D. A. (2010). Introduction to research in education. (9th ed.). Belmont, California: Wadsworth.

Berger, M. (2011, March 4). US employment picture at a glance. Retrieved from http://www.marketplace.org/topics/business/whiteboard/us-

Bowen, B. E., \& Lee, J. S. (1984). Education and occupational aspirations of students in agriculture majors. Journal of the American Association of Teacher Educators in Agriculture, 25(2), 28-29.

Bryan, W. J. The Biography.com website. Retrieved March 3, 2014, from: http://www.biography.com/people/william-jennings-bryan-922920.

Buckingham, M., \& Clifton, D. O. (2001). Now, discover your strengths. New York: The Free Press. Retrieved from http://www.amazon.com/Discover-Your-StrengthsMarcus-

Cole, L. (1985). Characteristics of those who select agricultural education as an undergraduate major at Oregon State University. Journal of American Association of Teacher Educators in Agriculture, 25(3), 79-85.

Donnermeyer, J. F., \& Kreps, G. M. (1994). Assessing college of agriculture freshmen. NACTA Journal, 38(1), 45-48.

Faulks, F. G. (1969). Who holds the key to success in recruiting teachers? Agricultural Education Magazine, 41(7), 168.

Findlay, H. J., \& Rawls, W. J. (1984). Factors that influence agricultural career objectives among students attending historically black four-year institutions. Journal of the American Association of Teacher Educators in Agriculture, 25(1), 28-34.

Fishbein, M., \& Ajzen, I. (1975). Belief, attitude, intention and behavior. Reading, MA: Addison-Wesley Publishing.

Garton, B. L., \& Robinson, J. S. (2006, May). Tracking agricultural education graduates' career choice, job satisfaction, and employability skills. Proceedings of the American Association for Agricultural Education Research Conference, (552563) 
Goecker, A. D., Smith , P. G., Smith , E., \& Goetz, R. (2010). Employment opportunities for college graduates in food, renewable energy, and the environment. Retrieved from http://www3.ag.purdue.edu/USDA/employment/Pages/default.aspx

Goecker, A. D., Whatley, C. M., \& Gilmore, J. L. (1999). Employment opportunities for college graduates in the food \& agricultural science, United States, 2000-2005. United States Department of Agriculture and Purdue University.

Greenwald, A. G. (1989). Attitude structure and function. Hillsdale, NJ: Erlbaum Associates.

Herzberg, F. (1976). The managerial choice: To be efficient and to be human. Dow Jones-Irwin: Homewood, IL.

Krueger, D. E., \& Riesenberg, L. E. (1991). Careers in agriculture as perceived by high school juniors and seniors. Proceedings of the National Agriculture Education Research Meeting, Los Angeles, California.

Llopis, G. (2012, June 04). The top 9 things that ultimately motivate employees to achieve. Retrieved from http://www.forbes.com/sites/glennllopis/2012/06/04/top9-things-that-ultimately-motivate-employees-to-achieve/

Martin, A. J., Milne-Home, J., Barrett, J., Spalding, E. \& Jones, G. (2000). Graduate satisfaction with university and perceived employment preparation. Journal of Education and Work, 13(2), 201-213.

Maslow, A. H. (1953). A theory of human motivation. Psychological Review, 50, 370396.

Miller, W. W., Williams, D. L., \& Sprouse, D. W. (1984). Factors concerning students' perceptions of teaching vocational agriculture. The Journal of the American Association of Teacher Educators in Agriculture, 25(3), 13-21.

National Research Council (1996). Colleges of agriculture at the land grant universities: Public service and public policy. Washington, DC: National Academy Press.

$\mathrm{Ng}$, V. (2008, October 31). Top 10 factors that motivate employees. Retrieved from http://www.smbworldasia.com/en/content/top-10-factors-motivate-employees

Orthel, G. R., Sorensen, J. L., Lierman, S. R., \& Riesenberg, L. E. (1989). High school students' perceptions of agriculture and careers in agriculture. Proceedings of the $16^{\text {th }}$ National Agricultural Education Research Meeting, Orlando, Florida.

Pink, D. (2009).Drive: The surprising truth about what motivates us. New York: Riverhead Books. 
Pink, D. H. (2008). The adventures of Johnny Bunko: The last career book you'll ever need. New York: Riverhead Books. Retrieved from http://www.amazon.com/TheAdventures-Johnny-Bunko-Career/dp/1594482918

Rawls, W. J., Martin, A., Negatu, S., \& Robertson, M. (1994). Educational plans of minority student participants in a university food and agricultural sciences recruitment program. NACTA Journal, 38(4), 15-19.

Reis, R., \& Kahler, A. A. (1997). Factors influencing enrollment in agricultural education programs as expressed by Iowa secondary agricultural education students. Journal of Agricultural Education, 38(2), 38-48.

Robinson, J. P., Shaver, P. R., \& Wrightsman, L. S. (1991). Criteria for scale selection and evaluation. In J. P. Robinson, P. R. Shaver, \& L. S. Wrightsman (Eds.). Measures of personality and social psychological attitudes (pp. 1-16) New York: Academic Press.

Scanlon, D. C., Bruening, T. H., \& Cordero, A. (1996). An industry perspective on changes needed in agricultural education curricula. Journal of Agricultural Education, 37(2), 17-23.

Spector, P. E. (2003). Industrial and organizational psychology: Research and practice. (3rd ed., pp. 190-191). Hoboken, NJ: John Wiley \& Sons, Inc.

Sutphin, H. D., \& Newsom-Stewart, M. (1995). Student's rationale for selection of agriculturally related courses in high school by gender and ethnicity. Journal of Agricultural Education, 36(2), 54-61.

Thompson, S. (1994). May the force be walloped. Vocational Education Journal, February, 21-23.

Welds , K. (2012). Lessons learned, do-over wishes: Regrets in life, career. Retrieved from http://kathrynwelds.com/tag/deloitte-shift-index-2012/

Wildman, M. \& Torres, R. (2001). Factors identified when selecting a major in agriculture. Journal of Agricultural Education, 42(2), 46-55.

The World Bank Group (2014). Employment in agriculture (\% of total employment). Retrieved from http://data.worldbank.org/indicator/SL.AGR.EMPL.ZS/countries/1WUS?display $=$ default 
APPENDICES 
APPENDIX A

Cover Letter 
April 1, 2014

Dear Davis of Agriculture, Natural Resources and Design Students:

As a student of the Davis College, you have made the choice to further your education with the hopes of a career in your intended field. Many factors may have played into your career decision. Some of these motivations may be based on society, others on your demographics. In order to discover the motivations behind your choice to choose a career in an agriculturally related field, your input would be greatly appreciated. You must be 18 years of age to participate in this study.

My name is Lacey Sims, a graduate student at West Virginia University. I am working with my advisor Dr. Deborah Boone to conduct a study on motivational factors leading to career choices in agriculturally related fields. This study will attempt to gather information on the factors that encourage students to choose careers in agriculturally related fields. The results of this study will be used to prepare a thesis to partially fulfill the requirements for a Master of Science degree in Agricultural and Extension Education.

Participation in this research study is completely voluntary and all information you provide will be held as confidential as possible. The questionnaire should take approximately ten minutes of your time to complete. You may skip any question you do not feel comfortable answering and may stop at any point and submit a partially completed survey. The responses to this survey will be reported in a summary format and individual responses will not be identifiable. Please answer questions honestly and to the best of your abilities. There is no penalty if you choose not to participate.

The online survey can be accessed through the following site: https://www.surveymonkey.com/s/2WC2Y7F After completing the survey click on the "done" button. The Institutional Review Board (IRB) at West Virginia University has approved this study and the acknowledgement of this research is on file.

We would like to thank you in advance for taking time to participate in this survey. Please submit the completed the survey by Tuesday, April 15, 2014. If you have questions please contact Lacey Sims at lsims2@mix.wvu.edu or Dr. Deborah Boone at 304-293-5450 or debby.boone@mail.wvu.edu.

Sincerely,

Lacey Sims

Graduate Student
Deborah A. Boone, Ph.D.

Associate Professor 
APPENDIX B

\section{Follow-Up Cover Letter}




\section{Dear Davis College Student,}

Last week you received a request to participate in a study regarding factors related to your decision to pursue a degree from the Davis College. If you have already completed that survey we thank you for your input. If you have not completed the survey, we hope you will go to the following site: https://www.surveymonkey.com/s/2WC2Y7F and complete the survey and assist a fellow student in their graduate research.

As a student of the Davis College, you have made the choice to further your education with the hopes of a career in your intended field. Many factors may have played into your career decision. Some of these motivations may be based on society, others on your demographics. In order to discover the motivations behind your choice to choose a career in an agriculturally related field, your input would be greatly appreciated. You must be 18 years of age to participate in this study.

My name is Lacey Sims, a graduate student at West Virginia University. I am working with my advisor Dr. Deborah Boone to conduct a study on motivational factors leading to career choices in agriculturally related fields. This study will attempt to gather information on the factors that encourage students to choose careers in agriculturally related fields. The results of this study will be used to prepare a thesis to partially fulfill the requirements for a Master of Science degree in Agricultural and Extension Education.

Participation in this research study is completely voluntary and all information you provide will be held as confidential as possible. The questionnaire should take approximately ten minutes of your time to complete. You may skip any question you do not feel comfortable answering and may stop at any point and submit a partially completed survey. The responses to this survey will be reported in a summary format and individual responses will not be identifiable. Please answer questions honestly and to the best of your abilities. There is no penalty if you choose not to participate.

The online survey can be accessed through the following site: https://www.surveymonkey.com/s/2WC2Y7F After completing the survey click on the "done" button. The Institutional Review Board (IRB) at West Virginia University has approved this study and the acknowledgement of this research is on file.

We would like to thank you in advance for taking time to participate in this survey. Please submit the completed the survey by Wednesday, April 16, 2014. If you have questions please contact Lacey Sims at 1sims2@mix.wvu.edu or Dr. Deborah Boone at 304-293-5450 or debby.boone@mail.wvu.edu.

Sincerely,

Lacey Sims

Graduate Student 
APPENDIX C

Third Follow-Up Cover Letter 


\section{Dear Davis College Student,}

This is the third request for you to participate in a research project for a fellow student about factors that influenced you to pursue a degree from the Davis College. If you have already completed that survey we thank you for your input. If you have not completed the survey, please go to the following site: https://www.surveymonkey.com/s/2WC2Y7F and complete the survey by April 13, 2014 and assist a fellow student in their graduate research.

As a student of the Davis College, you have made the choice to further your education with the hopes of a career in your intended field. Many factors may have played into your career decision. Some of these motivations may be based on society, others on your demographics. In order to discover the motivations behind your choice to choose a career in an agriculturally related field, your input would be greatly appreciated. You must be 18 years of age to participate in this study.

My name is Lacey Sims, a graduate student at West Virginia University. I am working with my advisor Dr. Deborah Boone to conduct a study on motivational factors leading to career choices in agriculturally related fields. This study will attempt to gather information on the factors that encourage students to choose careers in agriculturally related fields. The results of this study will be used to prepare a thesis to partially fulfill the requirements for a Master of Science degree in Agricultural and Extension Education.

Participation in this research study is completely voluntary and all information you provide will be held as confidential as possible. The questionnaire should take approximately ten minutes of your time to complete. You may skip any question you do not feel comfortable answering and may stop at any point and submit a partially completed survey. The responses to this survey will be reported in a summary format and individual responses will not be identifiable. Please answer questions honestly and to the best of your abilities. There is no penalty if you choose not to participate.

The online survey can be accessed through the following site: https://www.surveymonkey.com/s/2WC2Y7F After completing the survey click on the "done" button. The Institutional Review Board (IRB) at West Virginia University has approved this study and the acknowledgement of this research is on file.

We would like to thank you in advance for taking time to participate in this

survey. Please submit the completed the survey by Wednesday, April 23, 2014. If you have questions please contact Lacey Sims at 1sims2@mix.wvu.edu or Dr. Deborah Boone at 304-293-5450 or debby.boone@mail.wvu.edu.

Sincerely,

Lacey Sims

Graduate Student 
APPENDIX D

Final Cover Letter 
If you have already completed this survey we thank you for your input.

If you have not completed the survey, please go to the following site: https://www.surveymonkey.com/s/2WC2Y7F and complete the survey by April 30, 2014and assist a fellow student in their graduate research.

As a student of the Davis College, you have made the choice to further your education with the hopes of a career in your intended field. Many factors may have played into your career decision. Some of these motivations may be based on society, others on your demographics. In order to discover the motivations behind your choice to choose a career in an agriculturally related field, your input would be greatly appreciated. You must be 18 years of age to participate in this study.

My name is Lacey Sims, a graduate student at West Virginia University. I am working with my advisor Dr. Deborah Boone to conduct a study on motivational factors leading to career choices in agriculturally related fields. This study will attempt to gather information on the factors that encourage students to choose careers in agriculturally related fields. The results of this study will be used to prepare a thesis to partially fulfill the requirements for a Master of Science degree in Agricultural and Extension Education.

Participation in this research study is completely voluntary and all information you provide will be held as confidential as possible. The questionnaire should take approximately ten minutes of your time to complete. You may skip any question you do not feel comfortable answering and may stop at any point and submit a partially completed survey. The responses to this survey will be reported in a summary format and individual responses will not be identifiable. Please answer questions honestly and to the best of your abilities. There is no penalty if you choose not to participate.

The online survey can be accessed through the following site: https://www.surveymonkey.com/s/2WC2Y7F After completing the survey click on the "done" button. The Institutional Review Board (IRB) at West Virginia University has approved this study and the acknowledgement of this research is on file.

We would like to thank you in advance for taking time to participate in this

survey. Please submit the completed the survey by Wednesday, April 23, 2014. If you have questions please contact Lacey Sims at 1sims2@mix.wvu.edu or Dr. Deborah Boone at 304-293-5450 or debby.boone@mail.wvu.edu.

Sincerely,

Lacey Sims

Graduate Student 
APPENDIX E

Survey 


\section{Motivations for Careers in Agriculturally Related Fields}

Dear Davis College of Agriculture, Natural Resources and Design Students:

As a student of the Davis College, you have made the choice to further your education with the hopes of a career in your intended field. Many factors may have played into your career decision. Some of these motivations may be based on society, others on your demographics. In order to discover the motivations behind your choice to choose a career in an agriculturally related field, your input would be greatly appreciated.

My name is Lacey Sims, a graduate student at West Virginia University. I am working with my advisor Dr. Deborah Boone to conduct a study on motivational factors leading to career choices in agriculturally related fields. This study will attempt to gather information on the factors that encourage students to choose careers in agriculturally related fields. The results of this study will be used to prepare a thesis to partially fulfill the requirements for a Master of Science degree in Agricultural and Extension Education.

Participation in this research study is completely voluntary and all information you provide will be held as confidential as possible. The questionnaire should take approximately ten minutes of your time to complete. You may skip any question you do not feel comfortable answering and may stop at any point and submit a partially completed survey. The results of this survey will be reported in a summary format and individual responses will not be identifiable.

The Institutional Review Board (IRB) at West Virginia University has approved this study and the acknowledgement of this research is on file. If you have questions or concerns about completing the survey or participating in the study, please contact me at Isims2@mix.wvu.edu or my advisor Dr. Deborah Boone at debby.boone@mail.wvu.edu or 304-293-5450.

Thank you in advance for your participation in this study. We sincerely appreciate your time and assistance.

Sincerely,

Lacey Sims, Graduate Student

Deborah A. Boone, Ph.D., Associate Professor

\section{Do you anticipate pursuing an agriculturally related career?}

Yes

If No, what career do you plan to pursue?

Comment 
Motivations for Careers in Agriculturally Related Fields

2. Please indicate the level of influence each of the following has had on your decision to focus on an agriculturally related career. 1= Not at all Influential, 2=Mildly Influential, 3=Moderately Influential, 4=Highly Influential, NA= Not Applicable

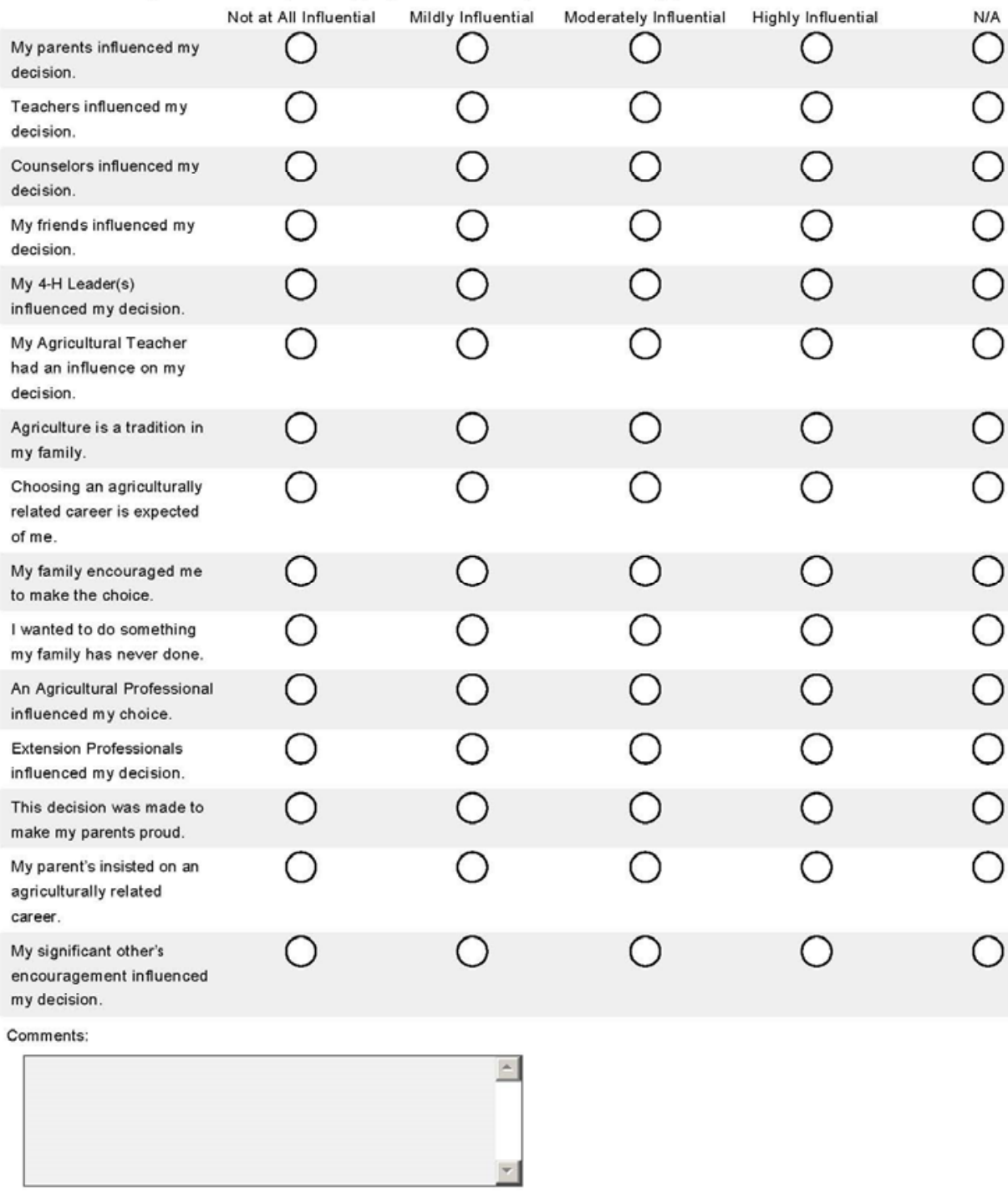

Page 2 


\section{Motivations for Careers in Agriculturally Related Fields}

3. Please indicate the level of influence each of the following has had on your decision to focus on an agriculturally related career.1= Not at all influential, 2 = Mildly Influential, 3 = Moderately Influential, 4 = Highly Influential, NA= Not applicable

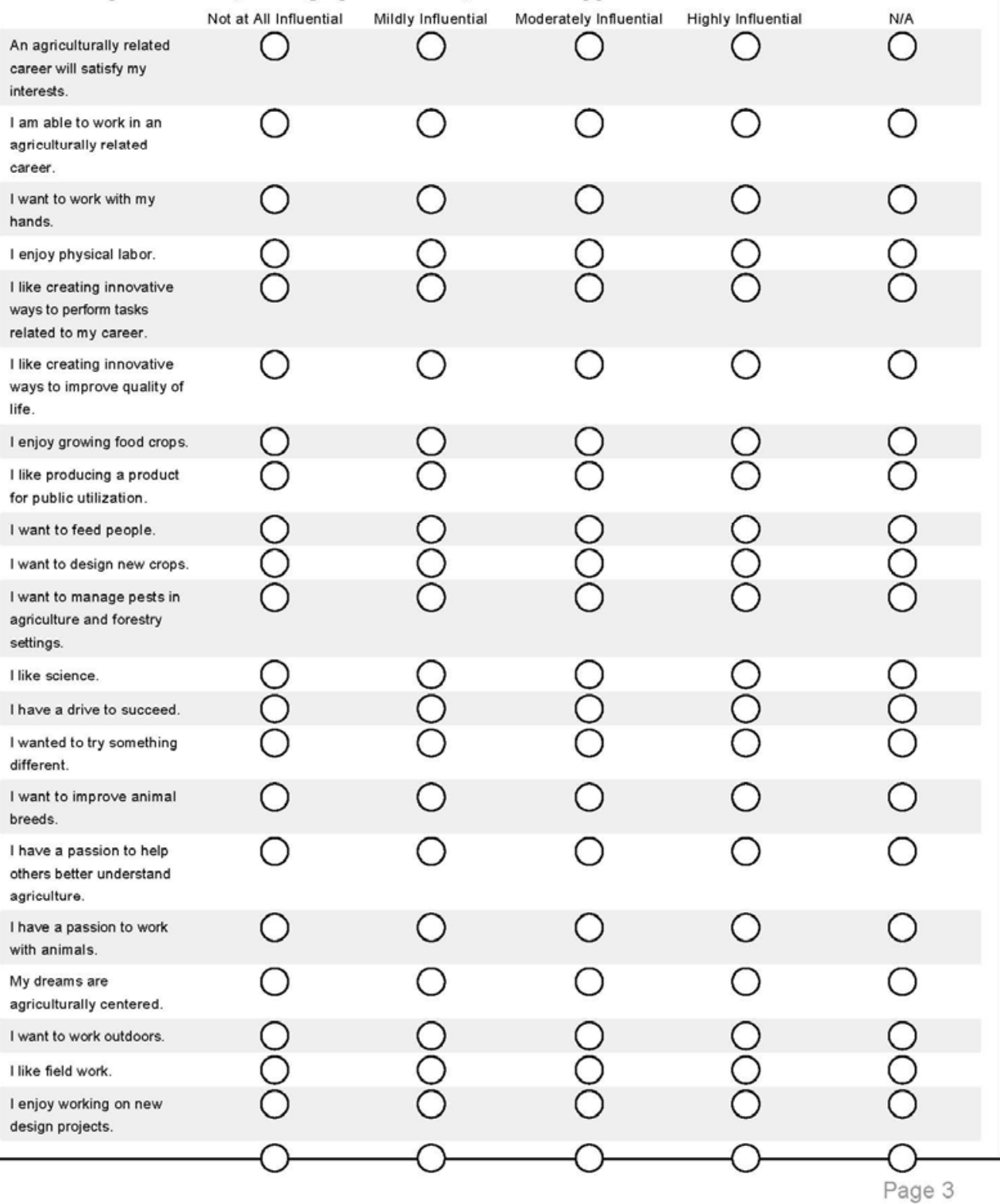




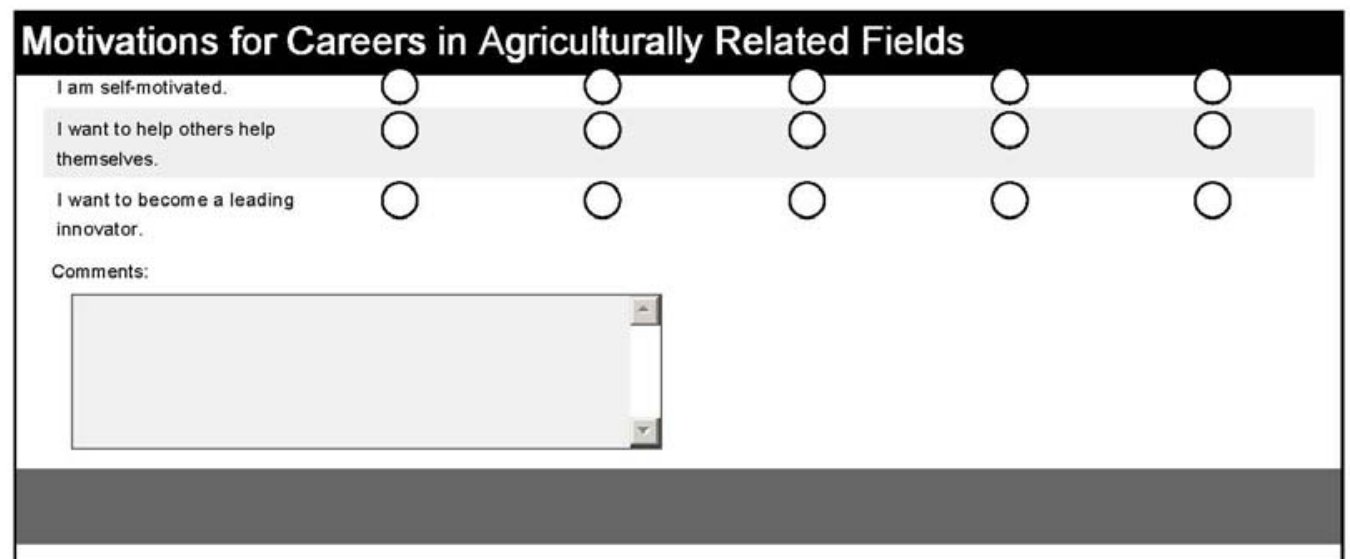

4. Please indicate the level of influence each of the following has had on your decision to focus on an agriculturally related career.1= Not at all influential, 2 = Mildly Influential, 3 = Moderately Influential, 4 = Highly Influential, NA= Not applicable

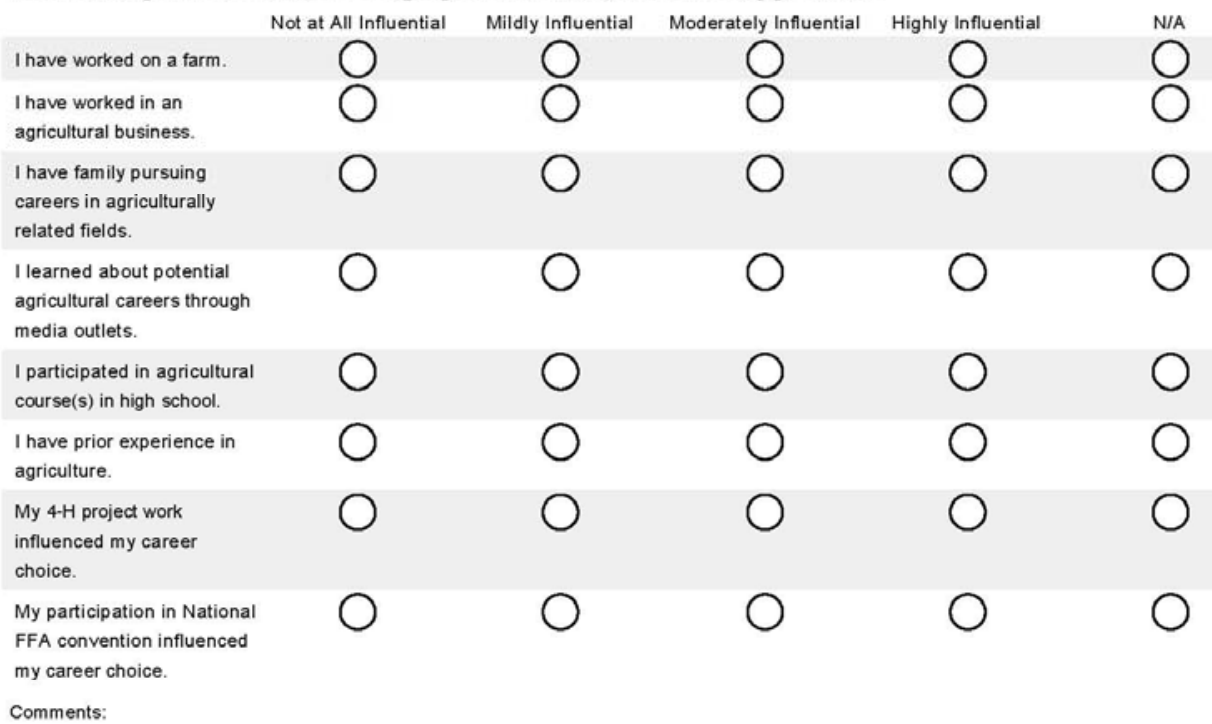

comments: 


\section{Motivations for Careers in Agriculturally Related Fields}

5. Please indicate the level of influence each of the following has had on your decision to focus on an agriculturally related career.1 $=$ Not at all influential, $2=$ Mildly Influential, 3 = Moderately Influential, 4 = Highly Influential, NA= Not applicable

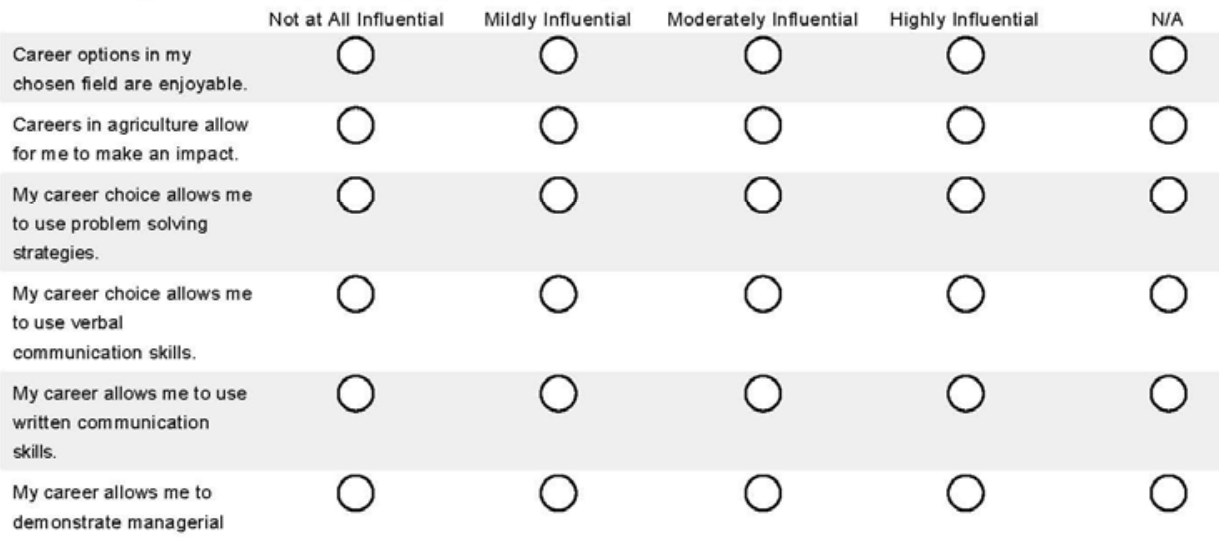

demonstrate managerial

skills.

My career allows me to

demonstrate leadership

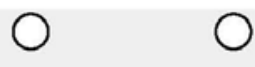

O

$0 \quad 0$

○

skills.

My career allows me to work

with people.

○

My career allows the

opportunity to work with

○

$\bigcirc$

O

O

$\bigcirc^{\text {N/A }}$

people of different cultures.

My career allows me to work

with environmental issues.

0

O

O

O

My career allows me to use

computer skills.

My career allows me to use

technical skills.

I want a career with room to

move-up the ladder.

My career provides the

opportunity to work with

highly knowledgeable

professionals.

My career choice offers a

variety of challenging

activities.

$\begin{array}{lll}0 & 0 & 0\end{array}$

○

O

O

○

O

O

0

O

O

0

0

0

0

0

$\mathrm{O}$

O

$\mathrm{O}$

O

O

O

O

Comments: 
Motivations for Careers in Agriculturally Related Fields

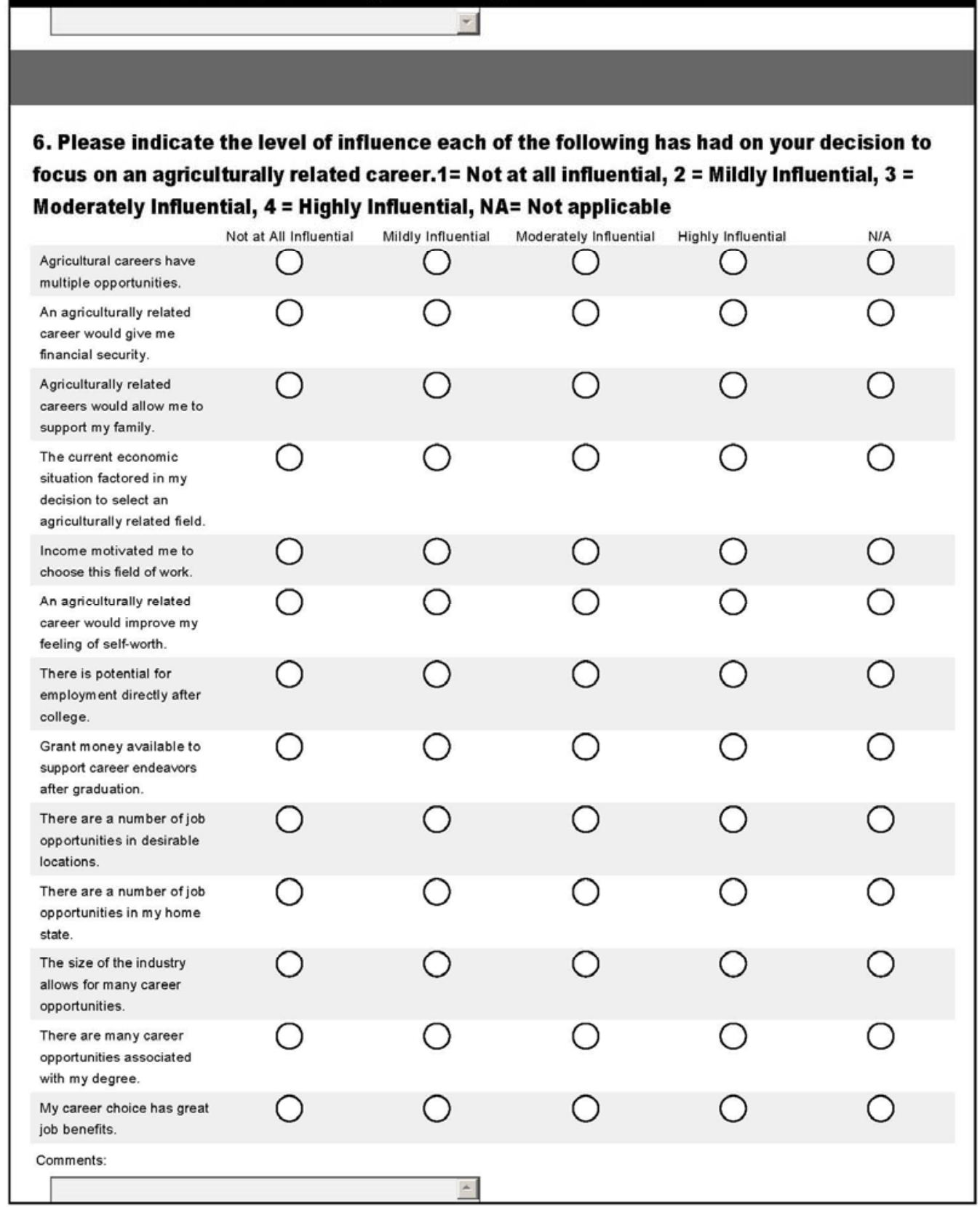


Motivations for Careers in Agriculturally Related Fields

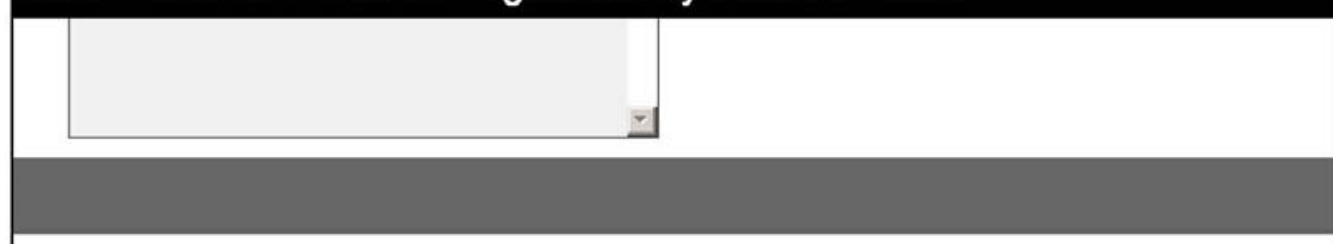

7. What is the estimated starting salary for your chosen field of work?
under $\$ 20,000$
○20,001-40,000
○ $\$ 40,001-60,000$
$\$ 60,001-80,000$
( $\$ 80,001-100,000$
\$100,001 and above

8. What is your mother/female guardian's occupation?

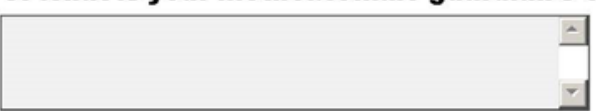

9. What is your father/ male guardian's occupation?

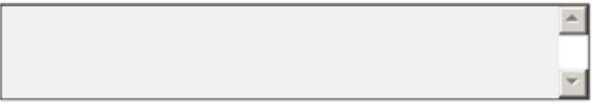

10. Please indicate the areas that best describe your prior agricultural background (Check all that apply).

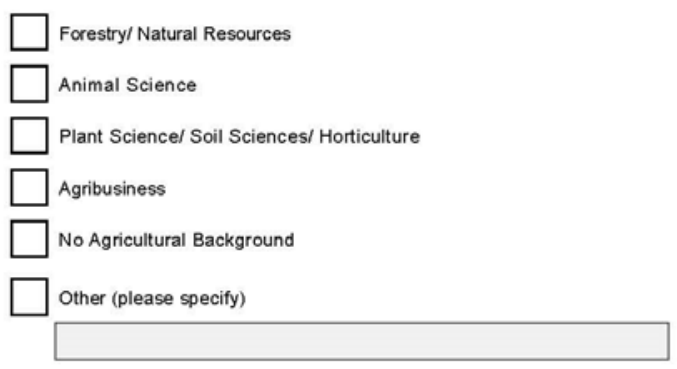


Motivations for Careers in Agriculturally Related Fields

11. Please indicate all the agriculturally related organizations in which you were involved as a youth? (Check all that apply)

$\square$ 4-H

$\square$ FFA (Future Farmers of America)

$\square$ Boy Scouts

$\square$ Girl Scouts

$\square$ Junior Breed Association

$\square$ Farm Bureau

Grange

Other (please specify)

-

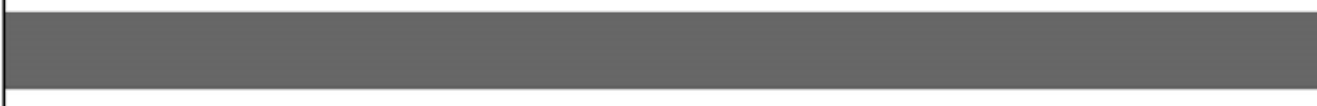

12. How satisfied are you with your current career choice?

very Satisfied

Satisfied

Dissatisfied

very Dissatisfied

Uncertain

13. What is your study focus at West Virginia University?

Study Focus

Other (please specify)

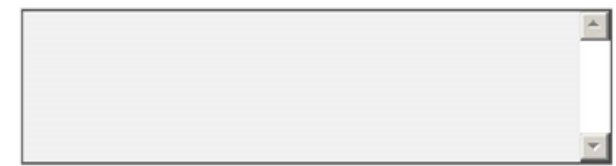

(20)

14. Are you a first generation college student?

Yes

No 
Motivations for Careers in Agriculturally Related Fields

15. Have you ever worked on a farm or ranch? (Excluding course work as a student on the University farm)

Ores

No

16. Have you ever worked in an agricultural business? (Excluding a farm or ranch)

Yres

№

17. Do you have relatives who live or work on a farm or ranch?

Yes

○o

18. Do you have relatives who work in an agricultural business? (Excluding a farm)

Yres

No

19. Did you take agricultural courses in high school?

Yres

No

20. Did your high school offer courses in agricultural education?

Ores

No

21. Since entering college at West Virginia University have you become involved in any agricultural organization(s)?

Yes

$\bigcirc$ No

If Yes, please explain 
Motivations for Careers in Agriculturally Related Fields

22. How would you describe your place of residence:

Small Farm/ Ranch

Rural Area, but not on a Farm or Ranch

Small Town; $<5,000$

Small City or Suburb; 5,000 to 50,000

Urban Area, City; $>50,000$

\section{What state do you consider home?}

State:

24. What is your gender?

Male

Female

25. What is your classification in college?

Freshman

sophomore

Junior

Senior

Graduate Student

Non-Degree seeking student

\section{What is your age?}

18 to 20

21 to 23

24 to 26

27 to 29

30 and up 


\section{Motivations for Careers in Agriculturally Related Fields}

27. What is your cumulative grade point average?

3.5-4.0

$3.0-3.49$

$2.5-2.9$

Below 2.49

Prefer not to respond

\section{Which category best describes your race?}

American Indian or Alaskan Native

Asian

Black or African American

Native Hawaiian or Other Pacific Islander

white

other

29. What is your ethnicity?

Hispanic or Latino

Non Hispanic or Latino

30. Do you have any other comments, questions, or concerns? 
APPENDIX F

What is your mother/ female guardian's occupation? 


\section{What is your mother/female guardian's occupation?}

Accountant (7)

Advertising

Agricultural business owner

Agriculture Research Associate

Art teacher

Assembler at a factory.

Author

Banker

Barber

Bookkeeper

Business management/ accounting and homemaking

Business Owner

Cashier

Certified Public Accountant in Ohio and Indiana. She currently works for Cassidy, Shiller and Associates.

Chief Compliance Officer for a hospital group

Chief Financial Officer

Civil engineer

Co-Business owner/ secretary

Construction cashier

Cosmetologist

Courthouse custodian

Craftsman

Deceased (4)

Dental assistant

Director of Human Resources

Director of public relations for a large Healthcare insurance company

Disabled (3)

Driver Supervisor at Pilgrim's Pride

Elementary school math teacher

EMT

Factory Worker (2)

Farmer

Field Trip Director

Food scientist

Foreign Church-planting Missionary

Government state department

Hairdresser and Homemaker

Head Cashier

Health and Licensing Inspector

High school teacher

Home care

Home maker (9)

House keeper (3) 
House wife (4)

Insurance

Insurance Agent

Insurance sales

Interior design

IT specialist

Janitor

Lab Technician

Lawyer

Legal Assistant

Librarian

Librarian

Line tech

Manager

Marketing

Medical coding professional

Medical Technologist

Microbiology scientist working for NOAA at a federal and state laboratory, she also

holds an administrative position

Mom works with state.

Mother is a Pediatric Nurse

Nanny

None (4)

Non-working

Not Applicable (4)

Nurse (9)

Optometrist

Owner of Eyewear Company

Owns/operates family Greenhouse

Painter

Parent liaison for public schools

Part sales clerk

Part time administrative assistant, part time farmer

Pediatric Palliative Care Nurse

Personal trainer

Personnel at Sam's Club

Physical therapist

Pickering Associates

Placement Specialist for the VA

Preschool teacher

Preschool teacher- speech pathologist

Principal of a high school

Professor

Rate Analyst

Realtor

Receptionist (2) 
Registered Nurse (2)

Research Assistant

Retired Administrative Assistant of Finances for the Hancock County, WV

Commissioners

$\mathrm{RN}$

School bus driver

School Cook

Secretary (4)

Secretary and Tax Preparer

Self-employed (2)

She is a poultry farmer. She produces and supplies poultry products to the local market.

She works in health care

Small business owner

Social Work (2)

Special Education

Statistical analyst

Stay at Home (5)

Stay at home mother, greenhouse manger

Substitute Teacher (3)

Substitute teachers aide

Supply chain and purchasing manager for ICL Chemical

Tax consultant

Teacher (17)

Teacher assistant

Teacher/professor

Teacher's Aide

Technical writer/project coordinator

Treasury management with first united nvm ank

Unemployed

Vet Office Secretary/Collections

Veterinarian technician

Waitress/bartender

Works for USDA

Works in school system

Youth service commission 
APPENDIX G

What is your father/ male guardian's occupation? 


\section{What is your father/ male guardian's occupation?}

Academy coordinator of a high school

$\mathrm{Ag}$ teacher farmer

Agricultural business owner

Agricultural Education Teacher

Agricultural research scientist, USDA

Animal Nutritionist and Crop Advisor

Auto part sales

Aviation Mechanic

Bartender/ Municipal Worker

Boat mechanic

Boss in the coal mines

Building Inspector

Business

Business owner

Business owner in oil and gas industry

Business owner/construction

Carpenter by trade, now works as a Director of the Labor Union

Chemical engineering

Chemical Operator

Chemical plant worker

Chemist (2)

Civil engineer (3)

Coal Miner (6)

Commercial Boat Captain/Salesman

Company Owner

Compressor operator

Construction (6)

Construction Contractor

Construction manager

Crew Chief/Supervisor at Mountaineer Gas Co.

Dad is a welder, Step dad is a cop

Deceased (3)

Defense Attorney

Defense contractor

Dental Technician

Department head at a (chocolate) factory

Department of justice

Director of Sales and Support for Trivantis, a computer training software company.

Doctor

Drilling rigs

Electrician (2)

Energy Management Technician

Engineer (4)

Environmental cleaning company management 
Environmental Scientist

Estate lawyer

Factory worker

Farm Bill Specialist for USDA NRCS

Farm owner/operator

Farmer

Farmer/bus driver

Father is an Artist

FedEx courier

Field technician

Finance (2)

First class line man

Foreign Church-planting Missionary

Franklin Township Supervisor

Full time farmer

Government (2)

Government Department of defense

Groundskeeper Owner/operator of family greenhouse

Heavy Equipment Operator (3)

High School Chemistry Teacher and Income Tax Business Owner

Horticulturalist (Tree Fruit Research Farm Manager)

Hydraulics

Insurance (previously was an extension agent)

Insurance Agent

IT Technician

Kroger employee

Lawyer (4)

Machine operator

Machinist

Maintenance and Farm Work

Maintenance man

Management at a store

Manager-car dealership

Managing a warehouse

Manufacturer (2)

Mechanic

Mechanic/Construction

Mechanical engineer

Mechanical equipment manager

Medical doctor

Metro driver

Military

Mining engineer

Minister (2)

NASA

Newspaper Editor 
None

Not Applicable (3)

NRCS

Oilfield Worker

Owner and operator of Sims Greenhouse Full time Beef cattle operation

Owner of newspaper

Park Ranger

Personal business owner

Pharmaceutical chemist

Pharmacist

Phone line man

Physician (3)

Physician assistant

Pipeline corrosion inspection and regulation

Plant manager/supervisor with shentel communications

Police officer (4)

President of transportation company

Professor

Public School Locksmith

Pump station engineer

Realtor and Land Development

Regional Supervisor of Parole and Probation

Research Assistant

Retired

Retired from the military but has two cash crop farms which he attends to.

Retired from the postal service

Retired from VA hospital/ maintenance

Retired Heavy Equipment Operator, WVDOH

Retired Science Teacher

Retired St. Superintendent

Retired, greenhouse manager

Road Construction Worker

Sales director

School bus driver

School system

Security Officer

Self-Employed plumber

Self-employed truck driver

Software developer

Sole proprietor and farmer

State Worker

Supervisor at a factory.

Surveyor 
Teacher (3)

Technical security specialist

Town administrator

Truck Driver (2)

Unemployed

UPS truck driver

USDA Farm Service Agency County Executive Director

USDA Forest Service

Veteran- Marine Corps US Postal Service Employee

Vice President of sales

VP of sales

VSST Assistant and Welding Instructor

Water Quality and Industrial Surveillance Manager

Welding- pipefitter

Williams Energy Project Manager

Worker

Works for USDA 
APPENDIX H

Agriculturally related organizations involved in as a youth - listed as other. 
Agriculturally related organizations involved in as a youth.

None in the US.

Rangers, STARS

Envirothon

I was not involved in any of these organizations

None

Envirothon

Young Life

Grange 


\section{APPENDIX I}

Study focus at West Virginia University? 'Other' Comments 


\section{Study focus at West Virginia University?}

Forest Resources Management

Religious Studies minor

Pre-medicine

Agribusiness Management Equine Management

Sustainable design minor Horticulture minor

Double Major Agribusiness Management and Rural Development along with

Horticulture

With a minor in Child Development and Family Studies

Horticulture with a minor in Landscape

Animal Nutritional Science

IPM and greenhouse management

And Agribusiness Management and Rural Development

My study focus is animal science. I hope to one day become a large animal veterinarian or do large animal research.

Forest resource management

Equine Management

And horticulture

Ag ED and ANS

Agribusiness management and rural development Minors in soil science and environmental economics 
APPENDIX J

Other comments, questions, or concerns? 


\section{Other Comments}

- I really feel that if someone is serious about a career in agriculture, they need to avoid West Virginia University.

- $\quad$ No (10)

- Even though I chose an "agricultural" degree, many of the questions were not relevant because I want to go into non-agricultural veterinary medicine.

- My biggest motivation choosing the Davis College was that they offered a Nutrition degree. I was diagnosed with Type 1 Diabetes before coming back to college, and wanted to learn about healthy foods and how our bodies use them. I want to help myself and others live healthfully and a healthy food supply is a big foundational aspect.

- I probably shouldn't have taken this....I'm sorry if I messed up your data.

- Hi Lacy!

- The most significant reason decided to become a teacher of agriculture is because feel that high school students who are interested in agriculture need more direction than what they are currently receiving. I want to make the agriculture education program for the students of high school age.

- Working outside helps keep me from getting depressed.

- Nope good luck lacy, Turk

- If I were to comment on everything this college has done to me, I would be here for a lifetime and $80 \%$ of it would be in the unsatisfactory category. 
VITA

Lacey J. Sims

Education:

August 2014

Masters of Science

Agricultural and Extension

Education

West Virginia University

Morgantown, WV

May 2013

Bachelors of Science

Agricultural and Extension Education

West Virginia University

Morgantown, WV

Professional Experience: June 2014 - August $2014 \quad$ Student Intern

Parkersburg Community

Foundation Fellowship

Program

Wirt County Extension

Office

Elizabeth, WV

September 2013 - May

2014

Receptionist

Morgantown Technical

Services Fabrication Shop

Morgantown, WV

May 2013 - August $2013 \quad$ Employee

Wards Farm Market

Kirk and Sharon Ward

Parkersburg, WV

January 2010 - May 2014 Student Intern

Sims Greenhouse

Bryan and Shari Sims

Palestine, WV 